NBER WORKING PAPER SERIES

\title{
EFFECTIVE DEMAND FAILURES AND THE LIMITS OF MONETARY STABILIZATION POLICY
}

\author{
Michael Woodford \\ Working Paper 27768 \\ http://www.nber.org/papers/w27768 \\ NATIONAL BUREAU OF ECONOMIC RESEARCH \\ 1050 Massachusetts Avenue \\ Cambridge, MA 02138 \\ September 2020, Revised September 2021
}

An earlier version was presented at the 2020 NBER Summer Institute under the title "Pandemic Shocks, Effective Demand, and Stabilization Policy." I would like to thank Aloísio Araújo, Gauti Eggertsson, Guido Lorenzoni, Argia Sbordone, Ludwig Straub, Harald Uhlig, and Iván Werning for helpful comments, and Yeji Sung for research assistance. The views expressed herein are those of the author and do not necessarily reflect the views of the National Bureau of Economic Research.

NBER working papers are circulated for discussion and comment purposes. They have not been peer-reviewed or been subject to the review by the NBER Board of Directors that accompanies official NBER publications.

(C) 2020 by Michael Woodford. All rights reserved. Short sections of text, not to exceed two paragraphs, may be quoted without explicit permission provided that full credit, including () notice, is given to the source. 
Effective Demand Failures and the Limits of Monetary Stabilization Policy

Michael Woodford

NBER Working Paper No. 27768

September 2020, Revised September 2021

JEL No. E12,E52,E63

\begin{abstract}
$\underline{\text { ABSTRACT }}$
The challenge for stabilization policy presented by the COVID-19 pandemic stems above all from disruption of the circular flow of payments, resulting in a failure of what Keynes (1936) calls "effective demand." As a consequence, economic activity in many sectors can be inefficiently low, and interest-rate policy cannot eliminate the distortions - not because of a limit on the extent to which interest rates can be reduced, but because interest-rate reductions fail to stimulate demand of the right sorts. Fiscal transfers are instead well-suited to addressing the fundamental problem, and can under certain circumstances achieve a first-best allocation of resources.
\end{abstract}

\author{
Michael Woodford \\ Department of Economics \\ Columbia University \\ 420 W. 118th Street \\ New York, NY 10027 \\ and NBER \\ mw2230@ columbia.edu
}


The COVID-19 pandemic has presented substantial challenges both to policymakers and to macroeconomists, and these go beyond the simple fact that the disturbance to economic life has been unprecedented in both its severity and its suddenness. The nature of the disturbance has also been different from those typically considered in discussions of business cycles and stabilization policy, and this has raised important questions about how to think about an appropriate policy response.

Among the more notable features of the economic crisis resulting from the pandemic has been the degree to which its effects have been concentrated in particular sectors of the economy, with some activities having to shut down completely for the sake of public health, while others continue almost as normal. A consequence of this asymmetry is a significant disruption of the "circular flow" of payments between sectors of the economy. In a stationary equilibrium of the kind to which an economy tends in the absence of shocks, each economic unit's payment outflows are balanced by its inflows, over any interval of time; this makes it possible for the necessary outflows to be financed at all times, without requiring the household or firm to maintain any large liquid asset balances.

Economic disturbances, regardless of whether these are "supply shocks" or "demand shocks," do not change this picture, as long as they affect all sectors of the economy in the same way: whether activity of all types is temporarily higher or lower, as long as the co-movement of the different sectors is sufficiently close, it continues to be the case that inflows and outflows should balance, so that financing constraints do not bind, even when many individual units maintain low liquid asset balances. Under such circumstances, the market mechanism should do a good job of ensuring an efficient allocation of resources. It is only necessary for policy to ensure that intertemporal relative prices (i.e., real interest rates) incentivize economic units to allocate expenditure over time in a way that is in line with variations in the efficient level of aggregate activity; in an economy where the prices of goods and services are fixed in advance in monetary units, this requires the central bank to manage the short-term nominal interest rate in an appropriate way. But it is often supposed that a reasonably efficient allocation of resources can be assured as long as interest-rate policy is adjusted in response to aggregate disturbances in a suitable way.

A disturbance like the COVID-19 creates difficulties of a different kind. The efficient level of some activities is now different, once public health concerns are taken into account. But in addition, the cessation of payments for the activities that are no longer safe interrupts the flow of payments that would ordinarily be used to finance other activities, even though these latter activities are still socially desirable (if one compares the utility that consumers can get from them to the disutility required to supply them). As a result, many activities may take place at a lower than efficient level, owing to insufficiency of what Keynes (1936) calls "effective demand" - the ability of people to signal in the marketplace the usefulness of goods to them, through their ability to pay for them. While it may well be efficient for restaurants or theaters to suspend the supply of their services for a period (because their usual customers cannot safely consume these services while the disease is rampant), the loss of their normal source of revenue may leave them unable to pay their rent; the loss of rental income may then require the real-estate management companies to dismiss their maintenance staff and fail to pay their property taxes; the furloughed maintenance staff may be unable to buy food or pay their own rent, the municipal government that does not receive a normal 
level of tax revenue may have to lay off city employees, and so on. ${ }^{1}$ The later steps in this chain of effects are all suspensions of economic transactions that are in no way required by the inability to continue supplying in-restaurant meals and theater performances.

An effective demand failure of this kind can result in a reduction in economic activity that is much greater than would occur in an efficient allocation of resources, even taking into account the public health constraint. Yet the problem is not simply that aggregate demand is too low, at existing (predetermined) prices, relative to the economy's aggregate productive capacity; in such a case one would expect the problem to be cured by a monetary policy that sufficiently reduces the real rate of interest. But as Leijonhufvud (1973) stresses, in a situation of sufficiently generalized effective demand failure, arising because financing constraints have temporarily become binding for a large number of economic units, the usual mechanisms of price adjustment in a market economy do not suffice to achieve an efficient allocation. The market-determined real rate of interest in a flexible-price economy will not achieve this; and neither, in the more realistic case of an economy with nominal rigidities, will a central bank that adjusts its policy rate to bring about the real rate of interest that would be associated with a flexible-price equilibrium, be able to do so.

Here we present a simple (and highly stylized) model to illustrate the nature of the problem presented by a disturbance like the COVID-19 pandemic. In our model, the fact that economic activity is much lower than in an optimal allocation of resources, in the absence of any policy response, does not necessarily imply that interest rates need to be reduced. While the model is one in which (owing to nominal rigidities) a reduction of the central bank's policy rate increases economic activity, the particular ways in which it increases activity need not correspond at all closely with the particular activities that it would most enhance welfare to increase. Instead, fiscal transfers directly respond to the fundamental problem preventing the effective functioning of the market mechanism, and can bring about a much more efficient equilibrium allocation of resources, even when they are not carefully targeted. And when fiscal transfers of a sufficient size are made in response to the pandemic shock, there is no longer any need for interest-rate cuts, which instead will lead to excessive current demand.

We are not the first to note that a crucial feature of the COVID-19 pandemic has been the degree to which its effects are sectorally concentrated; in particular, this is emphasized by both Guerrieri et al. (2020) and Baqaee and Farhi (2020). Indeed, the framework used here to consider alternative possible responses to a pandemic owes much of its structure to the pioneering work of Guerrieri et al. The emphases here are somewhat different, however, than in either of those earlier studies. We abstract altogether from either preference-based or technological complementarities between sectors, of the kind emphasized in the papers just cited, in order to focus more clearly on the consequences of the network structure of payments even in the absence of those other reasons for spillovers between activity in different sectors of the economy to exist. Because a key issue examined here is the effects of different possible network structures of payments, we consider a model in which there can be more than two sectors (and hence more than one sector still active in the case of a pandemic), unlike the baseline model of Guerrieri et al. And unlike either of these papers, we do not

\footnotetext{
${ }^{1}$ See, for example, Goodman and Magder (2020) and Gopal (2020) on the problems created by effective demand failures of this kind in New York City during the current crisis.
} 
assume that all consumers choose to consume the same basket of goods; as we show below, non-uniformity in the way expenditure is allocated across goods by economic units that also have different sources of income can play an important role in amplifying the magnitude of the effective demand shortfall resulting from a pandemic. ${ }^{2}$

The macroeconomics of a shock like COVID-19 is the subject of a rapidly expanding literature, already too large to easily summarize. Many interesting contributions focus on different issues than those of concern here. For example, Bigio et al. (2020) do not consider what can be achieved with conventional interest-rate policy, instead comparing the effects of lump-sum transfers with those of central-bank credit policies, in a model which emphasizes the endogeneity of borrowing limits (not analyzed here). Araújo and Costa (2021) similarly discuss how a modification of bankruptcy law in response to a pandemic shock can substitute for fiscal transfers. Caballero and Simsek (2020) consider the possible amplification of the effects of the shock through the effects of income reductions on endogenous financial constraints, from which we abstract here. Céspedes et al. (2020) primarily emphasize the longer-run costs of firms having to shed workers during the crisis; here we abstract from such effects, and show that transfers can be beneficial even when they are not taken into account. Auerbach et al. (2020) similarly emphasize the increased effects of transfer policies when there is endogenous exit of firms, and focus on channels through which transfers matter even in the absence of financing restrictions. None of these papers give much attention to the effects of conventional interest-rate policy in the case of a pandemic shock.

The paper proceeds as follows. Section 1 explains the structure of the model, and derives the first-best allocation of resources, both for the case of shocks that affect all sectors identically, and for asymmetric disturbances such as a pandemic shock. This section also shows that if there are only aggregate shocks, interest-rate policy suffices to achieve the first-best allocation as a decentralized equilibrium outcome, while lump-sum transfers are not only unnecessary, but also ineffective as a tool of aggregate demand management. Section 2 analyzes the effects of an asymmetric disturbance such as a pandemic shock in the absence of any monetary or fiscal policy response, showing how a collapse of effective demand can occurs. Section 3 shows how either fiscal transfers or government credit policy can mitigate the effects of such a disturbance by reducing the degree of effective demand shortfall, even when interest-rate policy does not respond to the shock at all; and shows that at least under some circumstances, the first-best allocation can be achieved without any change in interestrate policy. Finally, section 4 considers what can be achieved by adjusting the central bank's interest-rate target in response to the asymmetric disturbance, if the fiscal policy response is insufficient. It explains why this is more modest than might be expected, and offers examples in which ex-ante welfare is not improved by any reduction in interest rates at all, though the level of economic activity remains inefficiently low. Section 5 concludes.

\footnotetext{
${ }^{2}$ Other papers that stress the importance of network structure for the propagation of economic disturbances include Acemoglu et al., (2012), Bigio and La'O (2020), Elliott et al. (2021), Ghassibe (2021), La'O and Tahbaz-Salehi (2021), Ozdagli and Weber (2017), Pastén et al. (2020), and Rubbo (2020). These papers analyze the effects of production linkages between sectors (the input-output structure), which we abstract from here in order to emphasize the importance of the network structure of payments even in the absence of production linkages. Somewhat more closely related to our concerns here are the papers of Acemoglu et al. (2015) and Elliott et al. (2014), emphasizing the consequences of networks of financial obligations for the fragility of the financial system.
} 


\section{An $N$-sector Model}

Let us consider an $N$-sector "yeoman farmer" model, in which the economy is made up of producer-consumers that each supply goods or services for sale (subject to a disutility of supplying them), and also purchase and consume the goods or services supplied by other such units. Each such unit belongs to one of $N$ sectors (where $N \geq 2$ ) and specializes in the supply of the good produced by that sector, but consumes the goods produced by multiple sectors. ${ }^{3}$ We assume that there is a continuum of unit length of infinitesimal units in each of the sectors. We further order the sectors on a circle, and use modulo- $N$ arithmetic when adding or subtracting numbers from sectoral indices (thus "sector $N+1$ " is the same as sector 1 , "sector -2 " is the same as sector $N-2$, and so on).

\subsection{Preferences and the network structure of payments}

A producer-consumer in sector $j$ seeks to maximize the ex-ante expected value of a discounted sum of utilities

$$
\sum_{t=0}^{\infty} \beta^{t} U^{j}(t)
$$

where $0<\beta<1$ is a common discount factor for all sectors, and the utility flow each period is given by

$$
U^{j}(t)=\sum_{k \in K_{j}(t)} \theta_{k}^{j}(t) u\left(c_{k}^{j}(t) / \theta_{k}^{j}(t) ; \xi_{t}\right)-v\left(y_{j}(t) ; \xi_{t}\right),
$$

where $c_{k}^{j}(t)$ is the quantity consumed in period $t$ of the goods produced by sector $k$, and $y_{j}(t)$ is the unit's production of its own sector's good. The non-negative coefficients $\left\{\theta_{k}^{j}(t)\right\}$ allow a given sector to have asymmetric demands for the goods produced by the other sectors; $K_{j}(t)$ is the subset of subset of sectors $k$ for which $\theta_{k}^{j}(t)>0$ (so that $j$ wishes to consume goods produced in sector $k$ in period $t$ ). The vector $\xi_{t}$ represents aggregate disturbances that may shift either the utility from consumption or the disutility of supplying goods (or both $){ }^{4}$ note that these shocks are assumed to affect all goods and all consumers in the same way, as in standard one-sector New Keynesian models.

For any possible vector of aggregate shocks $\xi$, the utility functions are assumed to satisfy the following standard conditions: $u(0)=0$, and $u^{\prime}(c)>0, u^{\prime \prime}(c)<0$ for all $c>0$; $\lim _{c \rightarrow 0} u^{\prime}(c)=\infty$, and $\lim _{c \rightarrow \infty} u^{\prime}(c)=0$; and finally, $v(0)=0$, and $v^{\prime}(y)>0, v^{\prime \prime}(y) \geq 0$ for all $y>0$. The Inada conditions imply that the socially optimal supply of each good will be positive but finite (unless $\theta_{k}^{j}(t)=0$ for all $j$ in some period). Moreover, they imply that $\lim _{c \rightarrow \infty} u(c) / c=0$, so that $\theta u(c / \theta)$ has a well-defined limiting value (equal to 0 for any $c>0)$ as $\theta \rightarrow 0$. Hence our assumption in (1.2) that we simply omit terms for $k \notin K_{j}(t)$ results in a utility function that varies continuously with the coefficients $\left\{\theta_{k}^{j}(t)\right\}$.

\footnotetext{
${ }^{3}$ These "sectors" need not be interpreted as separate industries (e.g., travel and hospitality), though the direct impact of the COVID-19 shock was indeed extremely different across industries. They might equally well be understood as regions, or other ways in which economic units may be differentiated, that matter both for (i) the way they are impacted by some important disturbances, and (ii) the way in which other economic units allocate their spending.

${ }^{4}$ These may include aggregate productivity shocks, represented here as a shift in the disutility of effort required to produce a given quantity of output.
} 
Furthermore, the additively separable form (1.2) implies that closing down one sector (preventing either production or consumption of that good) has no effect on either the utility from consumption or disutility of supplying any of the other goods. Thus we abstract entirely from complementarities between sectors owing either to preferences or production technologies, of the kind stressed by Guerrieri et al. (2020), in order to focus more clearly on the linkages between sectors resulting from the circular flow of payments.

The coefficients $\left\{\theta_{k}^{j}(t)\right\}$ are important for our analysis, as they determine the network structure of the flow of payments in the economy; random variation in these coefficients is also the only kind of asymmetric disturbance that we consider. In this paper, we further specialize to the case in which $\theta_{k}^{j}(t)=\phi_{k}(t) \cdot \alpha_{k-j}$ for all $j, k$, and $t$. The only kind of random disturbance that we consider is a shock to the multiplicative factor $\phi_{k}(t)$ that affects the taste for sector $k$ 's products by everyone in the economy. (This allows us to consider a disturbance like the COVID-19 pandemic.) The constant coefficients $\left\{\alpha_{h}\right\}$ instead determine the degree to which the consumption preferences of units in different sectors $j$ are different. We assume that $\alpha_{h} \geq 0$ for each $h$, and also that $\alpha_{0}, \alpha_{1}>0$ (given an appropriate ordering of the sectors), to ensure that the network structure of payments is indecomposable. ${ }^{5} \mathrm{We}$ also assume that in any state of the world in any period $t, \phi_{k}(t) \geq 0$ for all $k$, and there is at most one sector $k$ for which $\phi_{k}(t)=0 .{ }^{6}$

We further normalize the $\left\{\alpha_{h}\right\}$ so that $\sum_{h=0}^{N-1} \alpha_{h}=1$. Then if $\phi_{k}(t)=1$ for each sector $k$ (which we will call the "normal case"), and in addition all goods have the same price in period $t$, the optimal intra-temporal allocation of expenditure by any sector $j$ will be given by

$$
c_{k}^{j}(t)=\alpha_{k-j} \cdot c^{j}(t)
$$

for each good $k$, where $c^{j}(t) \equiv \sum_{k=1}^{N} c_{k}^{j}(t)$ is total real expenditure by the sector in period $t$. Thus in this case the coefficients $\left\{\alpha_{h}\right\}$ correspond to expenditure shares. In the more general case where $\phi_{k}(t) \neq 1$ for one or more sectors (but still assuming that all goods have the same price), the optimal allocation of expenditure will be of the form

$$
c_{k}^{j}(t)=A_{k j}(t) \cdot c^{j}(t)
$$

where

$$
A_{k j}(t) \equiv \frac{\phi_{k}(t) \alpha_{k-j}}{\omega_{j}(\phi(t))}, \quad \omega_{j}(\phi(t)) \equiv \sum_{k} \phi_{k}(t) \alpha_{k-j} .
$$

(Our assumptions above guarantee that for any sector $j$ and any period $t$, there is at least one sector $k \in K_{j}(t)$, so that $\omega_{j}(\phi(t))>0$, and the coefficients $A_{k j}(t)$ are well-defined.)

The coefficients $\left\{\alpha_{h}\right\}$ are assumed to be the same for all sectors $j$; this means that in the "normal case", the model has a rotational symmetry: it is invariant under any relabeling of the sectors in which each sector $j$ is relabeled $j+r(\bmod N)$, for some integer $r$. Figure 1 illustrates two of the possible network structures allowed by our notation, for the case

\footnotetext{
${ }^{5}$ We exclude, for example, cases in which $N$ is even and even-numbered sectors purchase only from other even-numbered sectors, while odd-numbered sectors purchase only from odd-numbered sectors.

${ }^{6}$ Admitting the case in which $\phi_{k}(0)=0$ in some single sector $k$ allows us to consider the effects of a pandemic shock of the kind discussed by Guerrieri et al. (2020). We could also allow $\phi_{k}(0)$ to be zero in more than one sector, if we impose other restrictions that imply that the coefficients $\left\{\omega_{j}\right\}$ defined in (1.5) are nonetheless all positive.
} 

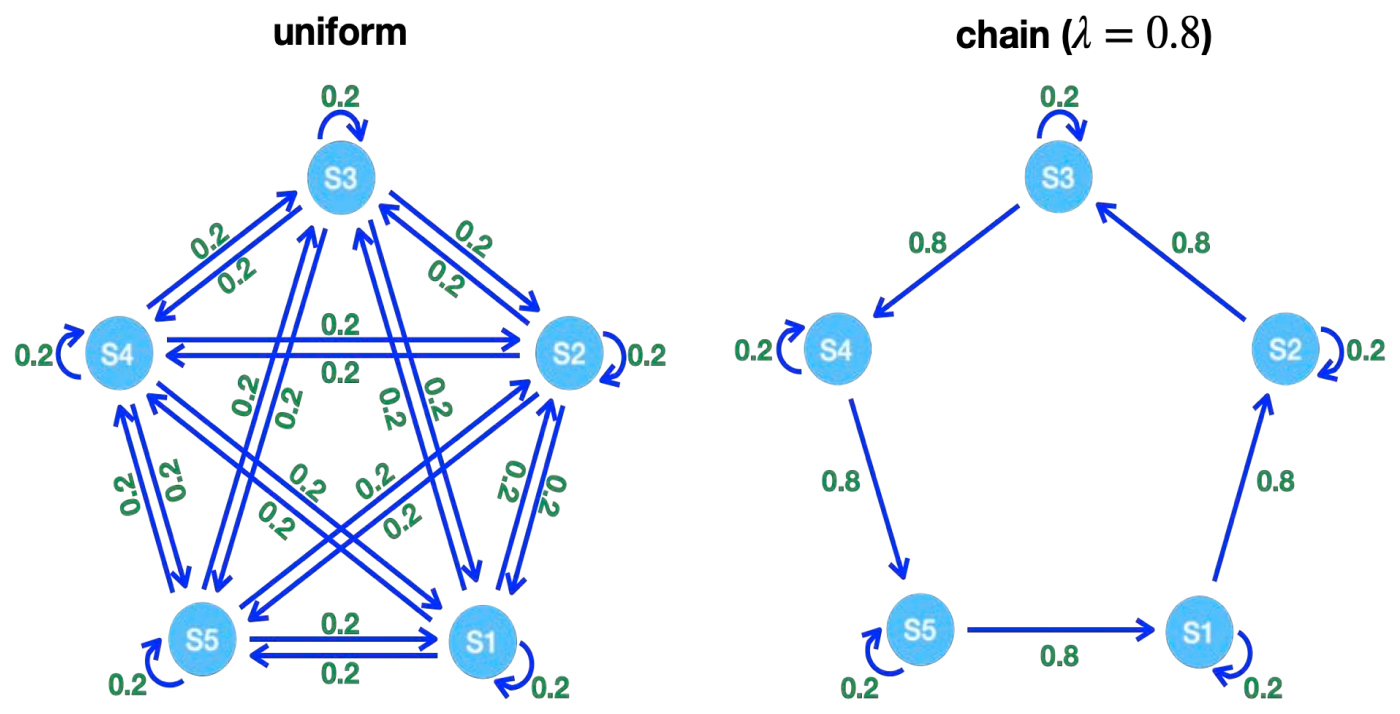

Figure 1: Two possible network structures when $N=5$. The number on the arrow from sector $j$ to sector $k$ indicates the value of the coefficient $\alpha_{k-j}$.

$N=5$. Note that in either case, the numbers on the arrows leaving any sector sum to 1; these indicate the share of that sector's spending allocated to each of the sectors to which arrows lead, in the "normal case" and when all goods prices are the same. Because of the rotational symmetry, the numbers on the arrows leading to any sector also sum to 1. If in addition to prices being the same, each sector spends the same amount, then all sectors' revenues will be the same, and each sector's inflows and outflows will be balanced. This illustrates the balanced "circular flow" of payments in an equilibrium in which only aggregate shocks occur (discussed further below).

The left panel shows the case of a uniform network, in which $\alpha_{h}=1 / N$ for all $h$. In this case, each sector has the same preferences over consumption bundles as any other sector, and these preferences treat all goods symmetrically; if the prices of all goods are the same, each individual unit will purchase the same quantity from each sector. The right panel instead shows the case of a "chain" network, in which $\alpha_{0}=1-\lambda$ and $\alpha_{1}=\lambda$, for some $0<\lambda<1$, while all other $\alpha_{h}$ are zero. In this case, each sector purchases only from its own sector and the sector immediately following it on the circle. In the numerical example shown in the figure, $\lambda=0.8$, so that in both examples the fraction of own-sector purchases (in the case that prices of all goods are equal) is the same (i.e., 20 percent). But in the left panel, outof-sector purchases are uniformly distributed over all of the other sectors, while in the right panel they are concentrated on one other sector. We show below that the network structure has important consequences for both the effects of a pandemic shock and the effects of fiscal transfers in response to the shock.

We further assume that the entire sequences $\xi \equiv\left\{\xi_{t}\right\}$ and $\phi \equiv\left\{\phi_{k}(t)\right\}$ for all periods $t \geq 0$ are revealed at time $t=0$; for simplicity, we suppose there is no further uncertainty about these disturbances to reveal after that. (This greatly simplifies our discussion of the short-run real effects of monetary policy.) We also assume for simplicity that asymmetric disturbances occur only in period $t=0$; that is, we assume that $\phi_{k}(t)=1$ for all $k$ in all 
periods $t \geq 1$. (Thus we analyze only the effects of unanticipated asymmetric disturbances.)

Finally, we assume that the ex ante distribution of possible realizations of $\phi(0)$ exhibits a rotational symmetry. Let $R$ be the matrix that maps an arbitrary $N$-vector $v$ to a vector $v^{\prime}=R v$, where $v_{k}^{\prime}=v_{k-1}$ for each $k$. Then we assume that any non-negative vector $\phi(0)$ has exactly the same ex-ante probability as the rotation $R \phi(0)$. (In the case of a pandemic shock that requires one sector to shut down while the others are unaffected, this assumption means that ex ante it is equally likely that any of the $N$ sectors could be the one impacted by the pandemic.) This means that despite our allowing for the possibility of asymmetric disturbances (such as a pandemic shock) in period zero, our model is rotationally symmetric ex ante. This is convenient both because it simplifies the solution for equilibrium outcomes, and because it provides us with an unambiguous ex ante welfare ranking of the outcomes associated with different stabilization policies, despite the differing situations of producerconsumers in the different sectors ex post.

\subsection{The first-best optimal allocation of resources}

As a benchmark for discussion of what stabilization policy can achieve, it is useful to define the first-best allocation that would be chosen by a social planner, given only the constraints of preferences and technology. For any possible realization of the disturbances $(\xi, \phi)$, let the set of $N$ possible rotations

$$
(\xi, \phi), \quad(\xi, R \phi), \ldots, \quad\left(\xi, R^{N-1} \phi\right)
$$

constitute the "rotation family" to which the particular realization $(\xi, \phi)$ belongs. $^{7}$ We can separately consider optimal policy for each possible rotation family.

We further consider only rotationally-invariant allocations of resources, that is, ones under which the consumption allocation $\left\{c_{k}^{j}(t ; \xi, \phi)\right\}$ associated with given disturbance sequences $(\xi, \phi)$ satisfies

$$
c_{k}^{j}(t ; \xi, R \phi)=c_{k-1}^{j-1}(t ; \xi, \phi)
$$

for all $j, k, t$ and any possible disturbance sequences $(\xi, \phi)$. Thus we do not allow policies that favor a particular sector, except to the extent that this results from the asymmetric impact of the exogenous disturbance $\phi$ on this sector; it must be the case that if the asymmetric disturbance were a rotation of the one that has actually occurred, the equilibrium allocation under the policy would have been correspondingly rotated. In the case of purely aggregate disturbances (the "normal case"), policies must treat units in all sectors identically. If we let $U^{j}(t ; \xi, \phi)$ be the flow utility (1.2) in the case of disturbances $(\xi, \phi)$ under such a policy, it follows that

$$
U^{j}(t ; \xi, R \phi)=U^{j-1}(t ; \xi, \phi)
$$

for each sector $j$.

Then considering only the possible outcomes associated with a particular rotation family (1.6), the terms in the ex ante expected value of (1.1) associated with these outcomes are

\footnotetext{
${ }^{7}$ Here for any sequence of $N$-vectors $\phi, \phi^{\prime}=R \phi$ means the alternative sequence such that $\phi^{\prime}(t)=R \phi(t)$ for each $t \geq 0$. Note that in the "normal case" in which $\phi_{k}(0)=1$ for all $k$, so that there is no asymmetric disturbance, the rotation family of the realization $(\xi, \phi)$ consists only of $(\xi, \phi)$ itself.
} 
proportional to

$$
\frac{1}{N} \sum_{h=0}^{N-1} \sum_{t=0}^{\infty} \beta^{t} U^{j}\left(t ; \xi, R^{h} \phi\right)=\frac{1}{N} \sum_{h=0}^{N-1} \sum_{t=0}^{\infty} \beta^{t} U^{j-h}(t ; \xi, \phi) .
$$

This sum is the same for each sector $j$, in addition to being the same for each of the sequences $(\xi, \phi)$ in a given rotation family. This immediately yields the following conclusion. ${ }^{8}$

Lemma 1. Units in all sectors $j$ agree about the ex ante ranking of alternative feasible rotationally-invariant allocations of resources. With regard to policy for a particular rotation family of possible disturbances, they unanimously prefer one allocation to another if in the case of any of the sequences $(\xi, \phi)$ of exogenous disturbances (once uncertainty is resolved at date $t=0)$ in this family, the first allocation achieves a higher value of

$$
\sum_{t=0}^{\infty} \beta^{t}\left[\sum_{j=1}^{N} U^{j}(t)\right]
$$

where $U^{j}(t)$ is defined in (1.2).

Given this, there is an obvious welfare objective to use in comparing alternative policies. We define the "first-best" optimal allocation in the case of disturbance sequences $(\xi, \phi)$ as the one that maximizes (1.7) under the constraints that $\sum_{j} c_{k}^{j}(t)=y_{k}(t)$ for each sector $k$ at each date $t$.

The welfare objective (1.7) can be written as a sum of separate terms for each good $k$ at each date $t$. We thus obtain a separate problem for each $k, t$ for which $\phi_{k}(t)>0$, in which we must choose $y_{k}(t)$ and the $\left\{c_{k}^{j}(t)\right\}$ for $j=1, \ldots, N$ to maximize

$$
\sum_{h \in H} \phi_{k}(t) \alpha_{h} u\left(c_{k}^{k-h}(t) /\left(\alpha_{h} \phi_{k}(t)\right) ; \xi_{t}\right)-v\left(y_{k}(t) ; \xi_{t}\right)
$$

where $H$ is the set of indices $h$ for which $\alpha_{h}>0$, subject to the constraints that $\sum_{j} c_{k}^{j}(t)=$ $y_{k}(t)$. (If $k$ is a sector such that $\phi_{k}(t)=0$, then the problem is trivial, and all quantities must equal zero.) The solution to this static problem is easily characterized.

Lemma 2. In the case of any disturbance sequences $(\xi, \phi)$, the unique first-best allocation of resources involves sectoral output levels $y_{k}(t)=y^{*}\left(\phi_{k}(t) ; \xi_{t}\right)$, where for any aggregate disturbance vector $\xi$ and any $\phi>0, y^{*}(\phi ; \xi)$ is the unique solution to the equation

$$
u^{\prime}\left(y^{*} / \phi ; \xi\right)=v^{\prime}\left(y^{*} ; \xi\right) .
$$

This supply of each good $k$ is then allocated to consumers according to

$$
c_{k}^{j}(t)=\alpha_{k-j} \cdot y_{k}(t)
$$

for each sector $j$.

\footnotetext{
${ }^{8}$ Proofs of all numbered lemmas and propositions are given in the online appendix.
} 
Thus the efficient level of output differs across sectors only as a result of asymmetry in the vector $\phi(0)$. In the "normal case" in which $\phi_{k}(0)=1$ for all $k$, and there are only aggregate shocks, the first-best allocation requires that $y_{k}(t)=y_{t}^{*}$ for each sector, where $y_{t}^{*}$ is defined by the same condition,

$$
u^{\prime}\left(y_{t}^{*} ; \xi_{t}\right)=v^{\prime}\left(y_{t}^{*} ; \xi_{t}\right)
$$

as determines the "natural rate of output" in the familiar one-sector model. In addition, in this case the optimal allocation shares are given by the coefficients $\left\{\alpha_{h}\right\}$, as in the examples in Figure 1.

In the case of an asymmetric disturbance to the vector $\phi(0)$, the optimal output in period zero differs across sectors. But it is only optimal for output to differ across sectors in the period in which the vector $\phi(t)$ is asymmetric (i.e., only in period $t=0$ ), and even in period $t=0$ it is only optimal for $y_{k}(0)$ to differ from $y_{0}^{*}$ to the extent that $\phi_{k}(0) \neq 1$ in that sector. Thus if we model a pandemic shock as a situation in which $\phi_{p}(0)<1$ in some sector $p$ only, while we continue to have $\phi_{k}(0)=1$ for all $k \neq p$, under the first-best resource allocation, output in sector $p$ should decrease (to a greater extent the greater the reduction in $\left.\phi_{p}(0)\right)$, while the efficient level of output in all sectors $k \neq p$ remains unchanged. ${ }^{9}$ In this respect, a pandemic shock can be considered a "negative supply shock," as in the discussion by Guerrieri et al. (2020).

\subsection{The decentralized economy}

Because all uncertainty is resolved at time $t=0$, the allocation of resources from period zero onward (conditional on the shocks revealed at that time) can be modeled as a perfect foresight equilibrium of a deterministic model. Each period, there are spot markets for the goods produced by all of the sectors for which $\phi_{k}(t)>0$, with $p_{k}(t)$ the money price of good $k$ in period $t$. There is also trading in a one-period nominal bond, that pays a nominal interest rate $i(t)$ between periods $t$ and $t+1{ }^{10}$ The price $p_{k}(t)$ of each sectoral good is assumed to be predetermined one period in advance, at a level that is expected at that earlier time to clear the market for good $k$ in period $t$; this temporary stickiness of prices allows monetary policy to affect real activity in period zero.

Let $a^{j}(t)$ be the nominal asset position of units in sector $j$ at the beginning of period $t$ (after any taxes or transfers), and $b^{j}(t)$ the nominal asset position at the end of the period (after period $t$ payments for goods are settled). Then in any period $t \geq 0$, a unit in sector $j$ chooses expenditures $\left\{c_{k}^{j}(t)\right\}$ (for $\left.k \in K_{j}(t)\right)$ and end-of-period assets $b^{j}(t)$ subject to the flow budget constraint

$$
\sum_{k \in K_{j}(t)} p_{k}(t) c_{k}^{j}(t)+b^{j}(t)=a^{j}(t)+p_{j}(t) y_{j}(t)
$$

and the borrowing constraint

$$
b^{j}(t) \geq \underline{b}^{j}(t)
$$

\footnotetext{
${ }^{9}$ See Figure 2 below for examples.

${ }^{10}$ Because there is only one possible future path for the economy conditional on the state in period zero, allowance for more than one financial asset in any period $t \geq 0$ would be redundant.
} 
where $y_{j}(t)$ is the quantity sold by the unit of its product, and $b^{j}(t) \leq 0$ is a (possibly sector-specific) borrowing limit.

The borrowing constraint (1.12) is crucial to our analysis, and in particular for the possibility of a collapse of effective demand, as analyzed in section 2 . We treat $\underline{b}^{j}(t)$ as a quantity determined by government policy (credit policy). We suppose that units are unable to credibly promise to repay, except to the extent that the government allows them to issue debt up to a certain limit, the repayment of which is guaranteed by the government. (We assume also that the government is able to force borrowers to repay these guaranteed debts, rather than bearing any losses itself.) Thus we shall refer to the case in which $\underline{b}^{j}(t)=0$ for all $j$ as the case of "no credit policy." 11

In period zero, $a^{j}(0) \geq 0$ is given as an initial condition for each sector; this quantity reflects not only wealth brought into the period (before shocks are realized), but also any transfers from the government in response to the shocks realized at time $t=0$, and the payoffs from any private insurance contracts conditional on those shocks. In any subsequent period, $a^{j}(t+1)$ is given by

$$
a^{j}(t+1)=(1+i(t)) b^{j}(t)-\tau(t+1) / N,
$$

where $\tau(t+1)$ is total lump-sum nominal tax collections at the beginning of period $t+1$, assumed to equally divided among all sectors. (We consider the possibility of sector-specific taxes or transfers only in period zero, in response to an asymmetric shock.) A unit in sector $j$ takes as given the value of $a^{j}(0)$, and the sequences $\left\{\xi_{t}, p_{k}(t), y^{j}(t), i(t), \tau(t+1)\right\}$ for all $t \geq 0$, and chooses sequences $\left\{c_{k}^{j}(t), b^{j}(t)\right\}$ consistent with constraints (1.11)-(1.13) for all $t \geq 0$ so as to maximize (1.1).

In equilibrium, the sales by units in each sector are given by

$$
y_{k}(t)=\sum_{j=1}^{N} c_{k}^{j}(t) .
$$

The assumption that prices are set in advance at a level expected to clear markets means that for each $j$, the sequence $\left\{y_{j}(t)\right\}$ for $t \geq 1$ must be the sequence that a unit in sector $j$ would choose, if it were also to choose that sequence at time $t=0$, taking as given the values of $a^{j}(0)$ and $y_{j}(0)$ and the sequences $\left\{\xi_{t}, p_{k}(t), y^{j}(t), i(t), \underline{b}^{j}(t), \tau(t+1)\right\}$ for all $t \geq 0$. The value of $y_{j}(0)$, however, need not be the one that units in sector $j$ would choose, given the shocks realized at $t=0$, because the price $p_{k}(0)$ is determined prior to the realization of those shocks. ${ }^{12}$ Because of the ex ante symmetry of our model, the predetermined prices $p_{k}(0)$ will all be set equal to some common price $\bar{p}>0$. The exact determinants of $\bar{p}$ are not relevant to our results below; it is only important that the price is the same for all sectors, and that it cannot be changed by any policy response to shocks realized at time $t=0$.

Conditions (1.11), (1.13) and (1.14) imply that the total supply of liquid assets $a(t)$ must evolve according to a law of motion

$$
a(t+1)=(1+i(t)) a(t)-\tau(t+1)
$$

\footnotetext{
${ }^{11}$ It is not essential to our conclusions that the borrowing limit be zero in the absence of government credit policy; what is important for our discussion below is that borrowing limits are unaffected by the changes in monetary or fiscal policy that we consider, other than an explicit change in credit policy.

${ }^{12}$ We suppose that when the pre-determined price $p_{k}(0)$ is set, the suppliers in sector $k$ agree that they will each supply an equal share of whatever quantity of good $k$ turns out to be demanded at that price.
} 
for all $t \geq 0$, which can be regarded as a flow budget constraint of the government. We shall consider only cases in which $a(t)>0$ for all $t \geq 0$, so that there exist public-sector liabilities, the interest rate on which is controlled by the central bank. ${ }^{13}$

We can now define an (ex-post) perfect foresight equilibrium given the exogenous disturbance sequences $(\xi, \phi)$ that have been realized in period zero, and the specifications of interest-rate policy, credit policy, and fiscal policy in response to such disturbances. This is a specification of the path of interest rates $\{i(t)\}$ for $t \geq 0$, prices $\left\{p_{k}(t)\right\}$ for $t \geq 1$, the allocation of resources $\left\{c_{k}^{j}(t), y_{k}(t)\right\}$ in each period $t \geq 0$, end-of-period balances $\left\{b^{j}(t)\right\}$ for periods $t \geq 0$, and associated beginning-of-period balances $\left\{a^{j}(t)\right\}$ in periods $t \geq 1$, such that (i) for each sector $j$, the plan specifying $\left\{c_{k}^{j}(t), b^{j}(t)\right\}$ for each $t \geq 0$ and $\left\{a^{j}(t), y_{j}(t)\right\}$ for each $t \geq 1$ maximizes (1.1), given $a^{j}(0)$ (determined by fiscal policy), $y_{j}(0)$ (determined by spending decisions in aggregate), the path of tax obligations $\{\tau(t)\}$ for $t \geq 1$ (determined by fiscal policy), the path of borrowing limits $\left\{\underline{b}^{j}(t)\right\}$ for $t \geq 0$ (determined by credit policy), and the endogenous paths of interest rates and prices; (ii) for each sector $k$, the quantity $y_{k}(t)$ produced satisfies (1.14) in each period $t \geq 0$; and (iii) total liquid asset holdings satisfy $\sum_{j} a^{j}(t)=a(t)$ in each period $t \geq 1$, where $\{a(t)\}$ is the path for the public debt determined by fiscal policy.

\subsection{Optimal policy if only aggregate shocks occur}

Let us consider first the "normal case" in which $\phi_{k}(0)=1$ for all $k$, but different sequences $\left\{\xi_{t}\right\}$ for the aggregate disturbances may be revealed in period $t=0$. Can the first-best allocation of resources (characterized above) be supported as an equilibrium, using only the policy instruments listed in the previous section? It is easily seen that this is possible, regardless of the predetermined price level $\bar{p}$ and the particular aggregate shock sequence $\left\{\xi_{t}\right\}$.

It suffices to consider policy regimes of the following kind. First, the central bank sets the interest rate in accordance with a Taylor rule of the form

$$
\log (1+i(t))=\log \left(1+r_{t}^{*}\right)+\pi^{*}(t+1)+\phi \cdot\left[\log (P(t) / P(t-1))-\pi^{*}(t)\right],
$$

for each $t \geq 0$, assuming that the right-hand side of the right-hand side of this formula is non-negative, and sets $i(t)=0$ otherwise. ${ }^{14}$ Here $r_{t}^{*}$ is the "natural rate of interest,"

$$
1+r_{t}^{*} \equiv \frac{1}{\beta} \frac{u^{\prime}\left(y_{t}^{*} ; \xi_{t}\right)}{u^{\prime}\left(y_{t+1}^{*} ; \xi_{t+1}\right)},
$$

a uniquely defined function of the exogenous disturbances; the price index $P(t)$ is equal to $(1 / N) \sum_{k} p_{k}(t)$; and $\left\{\pi^{*}(t)\right\}$ is a sequence of target inflation rates for $t \geq 0$. Second, the fiscal authority chooses period-zero transfers and a uniform tax obligation $\tau(t+1)$ each period

\footnotetext{
${ }^{13}$ See Woodford (2003, chap. 2) for discussion of the conduct of monetary policy by setting the nominal interest yield on an outside nominal asset of this kind.

${ }^{14}$ Here we assume that it is only feasible for the central bank to enforce a non-negative riskless nominal interest rate, though we do not model the institutional reasons for this to be the case (e.g., the possibility of storing non-interest-earning currency). But neither the assumption that the effective lower bound is exactly zero, nor even the assumption that there is a lower bound, matters for any of our conclusions below.
} 
after that so as to make (1.15) consistent with a target path $\{a(t)\}$ for the nominal public debt, where $a(t)>0$ each period, so that riskless debt exists in positive supply. Finally, there is no credit policy: $\underline{b}^{j}(t)=0$ each period for each sector $j$. A regime of this kind supports the first-best optimal allocation as an equilibrium outcome under the following conditions.

Proposition 1. Suppose that $\phi_{k}(0)=1$ for all $k$, so that only aggregate disturbances exist. And suppose (i) that interest-rate policy is determined by a Taylor rule (1.16), where the target inflation rate $\pi^{*}(0)$ is chosen to equal the predetermined inflation rate $\log (\bar{p} / P(-1))$, and subsequent targets are chosen so that

$$
\log \left(1+r_{t}^{*}\right)+\pi^{*}(t+1) \geq 0
$$

for all $t \geq 0$; (ii) that there is no credit policy; (iii) that there are no taxes or transfers in period zero (so that $a^{j}(0)=a(0) / N$ for each $j$ ); and (iv) that the target path $\{a(t)\}$ for the nominal public debt satisfies $a(t)>0$ for all $t \geq 0$, and

$$
\lim _{t \rightarrow \infty} \beta^{t} u^{\prime}\left(y_{t}^{*} ; \xi_{t}\right) \frac{a(t)}{P^{*}(t)}=0,
$$

where $\left\{P^{*}(t)\right\}$ is the path for the price level implied by the sequence of inflation targets (i.e., the requirement that $\log (P(t+1) / P(t))=\pi^{*}(t+1)$ for all $\left.t \geq 0\right)$ and the initial condition $P^{*}(0)=\bar{p}$. Then there exists an equilibrium in which the price of all goods is the same each period $\left(p_{k}(t)=P(t)\right.$ for all $\left.k\right)$; the central bank's inflation target is achieved each period $\left(P(t)=P^{*}(t)\right.$ for all $\left.t \geq 0\right)$; and the equilibrium allocation of resources is the first-best optimal allocation: $y_{k}(t)=y_{t}^{*}, c_{k}^{j}(t)=\alpha_{k-j} y_{t}^{*}$, for all $j, k$ in each period $t \geq 0$.

Note that under this optimal policy regime, neither the borrowing limits $\left\{\underline{b}^{j}(t)\right\}$, nor the initial sector-specific asset positions $\left\{a^{j}(0)\right\}$, nor the subsequent target path $\{a(t+1)\}$ for the public debt needs to respond to the particular sequence of aggregate disturbances $\left\{\xi_{t}\right\}$ that has been realized. Only the interest-rate reaction function (1.16) responds to the disturbances, through their effect on the implied path of the natural rate of interest. Thus all of the work of stabilization of the economy in response to exogenous disturbances is done by interest-rate policy, while fiscal policy is purely "passive."

Moreover, the model is one in which lump-sum transfers would not be useful for stabilization purposes. The equilibrium paths of both prices and quantities in Proposition 1 are the same, regardless of the exact path of the public debt $\{a(t)\}$. Thus making the path of lump-sum transfers and taxes state-contingent would have no effect, as long as we continue to assume that under any realization of the exogenous disturbances, (i) each sector $j$ receives the same transfers and is subject to the same tax liabilities, and (ii) the transversality condition (1.19) is satisfied. (Sector-specific transfers that redistribute income between sectors would change the equilibrium allocation of resources, but not in a way that increases the welfare objective $(1.7)$.)

Credit policy would similarly have no effect. We have written Proposition 1 to emphasize that the first-best can be achieved without credit policy; but we would obtain the same result in the case of any other paths $\left\{\underline{b}^{j}(t)\right\}$ for the borrowing limits, as long as $\underline{b}^{j}(t) \leq 0$ each period, and the borrowing limits satisfy a transversality condition similar to (1.19), to exclude the possibility of "Ponzi schemes." The reason is that as long as there are only aggregate 
disturbances, we have an equilibrium each period in which $p_{k}(t)=P(t)$ for each $k, c^{j}(t)$ is the same level of total spending for each sector, and each unit's expenditure allocation satisfies (1.3). It follows that each period we must have

$$
\sum_{k} p_{k}(t) c_{k}^{j}(t)=\sum_{k} p_{j}(t) c_{j}^{k}(t)=p_{j}(t) y_{j}(t)
$$

for each sector $j$, so that there is a balanced circular flow of payments (as in the examples in Figure 1). This in turn implies that asset balances remain uniformly distributed across sectors (given that we start from an initial uniform distribution), and that $b^{j}(t)=a^{j}(t)=$ $a(t) / N>0$ each period. Hence the borrowing limit never binds for units in any sector, and loosening the borrowing limits will not change any units' desired behavior, assuming that Ponzi schemes continue to be infeasible.

Thus in the case of only aggregate shocks (and fiscal policies that affect all sectors uniformly), fiscal transfers and credit policy are irrelevant as tools of stabilization policy, while monetary policy alone suffices to allow the first-best allocation of resources to be supported as an equilibrium outcome. As we shall see, our conclusions about the relative usefulness of these different types of policy are quite different in the case of a severely asymmetric disturbance, such as a pandemic.

In the case that there are only aggregate shocks, there is also a direct relationship between the degree to which output is above or below its efficient level and the degree to which monetary policy sets the interest rate at a level that is too low or too high, given the nature of the aggregate shock. This is illustrated by the following generalization of Proposition 1 .

Proposition 2. Suppose that $\phi_{k}(0)=1$ for all $k$, so that only aggregate disturbances exist. Suppose also that fiscal policy and credit policy are specified as in Proposition 1, and that interest-rate policy is the same as is specified in Proposition 1 for all periods $t \geq 1$, but that $i(0)$ need not satisfy (1.16). Then there is again an equilibrium in which $p_{k}(t)=P^{*}(t)$ for all $k$ and all $t$, and in which the allocation of resources in each period $t \geq 1$ is the one specified in Proposition 1. In period $t=0$, the allocation of resources continues to be rotationally symmetric:

$$
y_{k}(0)=y(0), \quad c_{k}^{j}(0)=\alpha_{k-j} y(0)
$$

for all $j, k$. However, the common level of sectoral output $y(0)$ will in general not equal $y_{0}^{*}$ (the efficient level, given the disturbance $\left.\xi_{0}\right)$. Instead, the equilibrium involves $y(0)<y_{0}^{*}$ if and only if $i(0)$ is higher than the value specified by (1.16), while $y(0)>y_{0}^{*}$ if $i(0)$ is lower than the right-hand side of (1.16).

This result implies that (under the conditions assumed in the proposition) an observation that output is inefficiently low in period $t=0$ (the period for which prices have been predetermined, before realization of the disturbance sequence $\xi$ ) implies that the interest rate is too high in that period, relative to the disturbance sequence that has occurred (which determines the value of $r_{0}^{*}$ ). This conclusion is independent of the exact specification of fiscal policy and credit policy, within a wide range of possible specifications of the latter policies. It suggests that a measure of the aggregate "output gap" might be enough to indicate that the central bank's interest-rate target should be reduced in response to a particular type of real disturbance, regardless of the nature of that disturbance. (The conclusion in Proposition 
2 holds whether $\xi_{0}$ represents a shock to the degree of impatience to consume, a shock to the disutility of working, or a shock to labor productivity, among other possibilities.) But as we shall see, the same is not true in the case of an asymmetric disturbance.

\section{Asymmetric Disturbances and Effective Demand Failure}

In the case of an asymmetric disturbance, the policy specified in Proposition 1 no longer suffices to ensure the existence of an equilibrium in which the allocation of resources is the first-best optimal allocation characterized in Lemma 2. One might suppose that this is because we have assumed that prices are fixed a period in advance, and the uniform prices $\left(p_{k}(0)=\bar{p}\right.$ for all $\left.k\right)$ that are chosen ex ante will in general imply the wrong relative prices ex post, in the case of an asymmetric disturbance. This is indeed generally an obstacle to attainment of the first-best allocation. But as shown below, there are cases in which the first-best allocation can be achieved using only the policy instruments specified in section 1.3 above, despite the occurrence of an asymmetric disturbance. Yet in these cases, not only is the optimal policy not the one specified in Proposition 1, it is not a policy under which interest-rate policy alone responds to the disturbance. Moreover, it is not necessarily even the case that a disturbance that results in an inefficiently low level of output implies that matters would be improved by reducing the interest rate.

\subsection{Equilibrium with an asymmetric disturbance}

We begin by discussing how equilibrium is different in the case of a disturbance that causes $\phi_{k}(0)$ to differ across sectors, such as a pandemic (modeled as an exogenous reduction of $\phi_{k}(0)$ in some, but not all sectors, owing to health concerns that temporarily preclude consumption of certain goods and services). Since aggregate disturbances create no problems that cannot be dealt with using monetary policy alone, as shown in Proposition 1, from here on we abstract from them: we assume that $\xi_{t}=\bar{\xi}$ for all $t \geq 0$, and let $\bar{y}=y^{*}(1 ; \bar{\xi})$ be the natural rate of output associated with these constant values for the disturbance parameters. ${ }^{15}$

Our primary interest is in the effects of alternative possible policy responses in period $t=0$ to asymmetric shock to the vector $\phi(0)$. Hence we shall simplify our discussion by assuming that policy from period $t=1$ onward is of a particular sort.

Assumption 1. In all periods $t \geq 1$, monetary and fiscal policy are assumed to satisfy conditions ( $i$ ) and (iv) of Proposition 1 in all periods. The borrowing limits satisfy $\underline{b}^{j}(t) \leq 0$ for all $j$ and all $t \leq 1$, together with the requirement that

$$
\lim _{t \rightarrow \infty} \beta^{t} \frac{\underline{b}_{t}^{j}}{P^{*}(t)}=0
$$

for each sector $j$. In addition, let

$$
\tilde{a}^{j}(1) \equiv(1+i(0)) \frac{b^{j}(0)}{P^{*}(1)}
$$

\footnotetext{
${ }^{15}$ It would be straightforward to generalize our results below to the case on non-zero aggregate shocks, at the cost of more complex formulas, but without changing our conclusions about the effects of shocks to $\phi(0)$.
} 
denote the real pre-tax wealth carried into period 1 by each of the units in sector $j$, if the period-1 inflation rate conforms to the central bank's target. Then the paths $\left\{a(t), \underline{b}^{j}(t)\right\}$ satisfy

$$
\frac{(1 / N) a(t)-\underline{b}^{j}(t)}{P^{*}(t)} \geq \beta\left[(1 / N) \sum_{\ell=1}^{N} \tilde{a}^{\ell}(1)-\tilde{a}^{j}(1)\right]
$$

for each $j$ and all $t \geq 1$.

Condition (2.20) implies that even if credit policy relaxes borrowing constraints for some or all sectors, the level of allowable borrowing does not grow so fast as to make a Ponzi scheme feasible: debt must eventually be repaid. On the other hand, condition (2.22) implies that borrowing limits will also not be contracted too quickly. While the condition is stated in Assumption 1 purely as a constraint on variables in periods $t \geq 1$, a sufficient condition for (2.22) to hold is that

$$
\left[\frac{(1 / N) a(t)-\underline{b}^{j}(t)}{P^{*}(t)}\right] \geq \beta(1+i(0)) \frac{P^{*}(0)}{P^{*}(1)}\left[\frac{(1 / N) a(0)-\underline{b}^{j}(0)}{P^{*}(0)}\right]
$$

for all $t \geq 1$. That is, the condition holds if we assume that in the case of any increase of aggregate liquidity $a(0)$ through fiscal transfers, or relaxation of the borrowing limit $\underline{b}^{j}(0)$ of any sector in period 0 will be permanent (in real terms). This implies that the government does not create liquidity difficulties for any sector in periods $t \geq 1$ by insisting that either public or private borrowing must be rapidly repaid.

Because we assume that economic fundamentals are the same in all periods $t \geq 1$, despite the occurrence of an asymmetric disturbance in period zero, it is natural to expect a perfect foresight equilibrium in which quantities and relative prices are constant for all $t \geq 1$. And because the equilibrium in periods $t \geq 1$ must be the same as if all goods prices were fully flexible, the resource allocation and relative prices in each of the periods $t \geq 1$ should correspond to those of a static (one-period) competitive equilibrium. Let $\boldsymbol{f}$ be a vector of net transfers to the units in the different sectors (where the element $f^{j}$ is the net transfer to sector $j$ ), measured in units of a composite good consisting of one unit of each of the $N$ goods, and such that $\sum_{j} f^{j}=0$. For any such vector, consider a one-period competitive equilibrium model in which units in each sector $j$ choose quantities $\left\{c_{k}^{j}\right\}$ and $y_{j}$ to maximize (1.2), subject to the budget constraint

$$
\sum_{h \in H} q_{j+h} c_{j+h}^{j}=q_{j} y_{j}+f^{j}
$$

where $q_{k} \equiv p_{k} / P$ is the relative price of good $k$. Competitive equilibrium requires that the relative prices be such that markets clear, i.e., such that (1.14) holds for each $k$.

Standard methods ${ }^{16}$ allow one to show that the collection of endowment vectors $\boldsymbol{f}$ and corresponding equilibrium relative prices $\boldsymbol{q}$ form a smooth manifold of dimension $N-1$. Because of the rotational symmetry of the static model, one point on this manifold must be $(\mathbf{0}, \overline{\boldsymbol{q}})$, where $\bar{q}_{k}=1 / N$ for all $k$, the equilibrium in which $c_{k}^{j}=\alpha_{k-j} \bar{y}, y_{k}=\bar{y}$ for all

\footnotetext{
${ }^{16}$ See, for example, Balasko (2009).
} 
$j, k .{ }^{17}$ It follows that for some neighborhood $U$ of the vector $\mathbf{0}$, we can define a continuously differentiable function $\boldsymbol{q}^{*}(\boldsymbol{f})$ such that $\boldsymbol{q}^{*}(\mathbf{0})=\overline{\boldsymbol{q}}$, and such that for any $\boldsymbol{f} \in U$, the pair $\left(\boldsymbol{f}, \boldsymbol{q}^{*}(\boldsymbol{f})\right)$ also belongs to the equilibrium manifold - that is, such that $\boldsymbol{q}^{*}(\boldsymbol{f})$ is an equilibrium vector of relative prices in the case of an endowment vector $\boldsymbol{f}$. In this way, for any endowment vector close enough to $\mathbf{0}$, we select a competitive equilibrium price vector that is correspondingly close to the uniform vector of relative prices $\overline{\boldsymbol{q}}$. Corresponding to any such equilibrium price vector there is also a uniquely defined competitive equilibrium allocation, given by the solution to the problem of maximizing (1.2) subject to the static budget constraint (2.23), for each sector $j$.

We can then use this solution for competitive equilibrium in the static model to define a perfect foresight equilibrium for periods $t \geq 1$, as a function of the net asset position of units in each of the sectors at the beginning of period $t=1$. Let $\tilde{\boldsymbol{a}}(1)$ be the vector specifying the net asset position of each of the sectors at the beginning of period 1 . Then as long as the vector $\tilde{\boldsymbol{a}}(1)$ indicates a wealth distribution at the beginning of period $t=1$ that is not too non-uniform, we can compute a perfect foresight equilibrium for periods $t \geq 1$ that depends only on the vector $\tilde{\boldsymbol{a}}(1)$.

Lemma 3. Let pre-tax net asset positions at the beginning of period $t=1$ be specified by a vector $\tilde{\boldsymbol{a}}(1)$, and suppose that the implied vector of sectoral net incomes

$$
f^{j}=(1-\beta)\left[\tilde{a}^{j}(1)-(1 / N) \sum_{\ell=1}^{N} \tilde{a}^{\ell}(1)\right]
$$

satisfies $\boldsymbol{f} \in U$, where $U$ is the neighborhood on which the static equilibrium selection is defined. Suppose furthermore that policy satisfies Assumption 1 in all periods $t \geq 1$.

Then there exists a flexible-price perfect-foresight equilibrium for periods $t \geq 1$, consistent with the assumed pre-tax net asset positions, of the following kind. Relative prices each period are given by $\boldsymbol{q}^{*}(\boldsymbol{f})$, and the allocation of resources each period is the stationary allocation that, for each sector $j$, solves the problem of maximizing (1.2) subject to the constraint (2.23), given these relative prices and the transfer $f^{j}$ specified in (2.24). The price index each period is given by the central bank's inflation target, $P(t)=P^{*}(t)$, and the real interest rate each period is given by

$$
(1+i(t)) \frac{P(t)}{P(t+1)}=1+r^{*}=\frac{1}{\beta}>1 .
$$

In this equilibrium, the borrowing constraint (1.12) never binds for units in any sector, in any period $t \geq 1$.

Finally, we can use this solution for the perfect foresight equilibrium for all periods $t \geq 1$, conditional on the assets carried into period 1, to characterize equilibrium behavior in period $t=0$ as well, as a function of the asymmetric disturbance. Suppose that the real pre-tax assets carried into period 1 by units in general are described by a vector $\tilde{\boldsymbol{a}}(1)$, but that an individual unit in sector $j$ contemplates carrying some other level of real pre-tax assets $\tilde{a}$ into period 1 . We can define the optimization plan for this individual unit from $t=1$ onward, if

\footnotetext{
${ }^{17}$ Note that this point corresponds to the stationary equilibrium in each period $t \geq 1$ in the intertemporal equilibria characterized in Propositions 1 and 2.
} 
its initial assets are $\tilde{a}$ and it faces the relative prices and real interest rates implied by the aggregate decisions $\tilde{\boldsymbol{a}}(1)$ (as characterized in Lemma 3 ).

Let $V^{j}(\tilde{a} ; \tilde{\boldsymbol{a}}(1))$ be the discounted utility flow for this individual unit in periods $t \geq 1$ (discounted back to period 1). The unit's optimization problem in period zero (after the realization of the disturbances and any policy response) can then be written as a choice of an expenditure plan $\left\{c_{k}^{j}(0)\right\}$ for all $k \in K_{j}(0)$ and end-of-period asset balance $b^{j}(0)$ consistent with (1.11)-(1.12), so as to maximize the objective

$$
U^{j}(0)+\beta V^{j}(\tilde{a} ; \tilde{\boldsymbol{a}}(1))
$$

where $U^{j}(0)$ is given by $(1.2)$ and $\tilde{a}=(1+i(0)) b^{j}(0) / P^{*}(1)$. This defines an optimization problem for units in any sector $j$ that depends on the period-zero policy variables $a^{j}(0)$ (reflecting possible transfers in response to the disturbance), $\underline{b}^{j}(0)$, and $i(0)$.

Given policy choices $\left\{a^{j}(0), \underline{b}^{j}(0), i(0)\right\}$, an equilibrium is then a plan $\left\{c_{k}^{j}(0), b^{j}(0)\right\}$ for units in each sector $j$, and a vector $\tilde{\boldsymbol{a}}(1)$, such that (i) for each $j$, the plan $\left\{c_{k}^{j}(0), b^{j}(0)\right\}$ solves the optimization problem for an individual unit (just stated), given the policy variables and the vector $\tilde{\boldsymbol{a}}(1)$ of aggregate decisions; and (ii) $\tilde{\boldsymbol{a}}(1)$ is the vector of sectoral net asset positions at the beginning of period 1 implied by these plans, using (2.21).

Moreover, the optimal plan for each sector $j$ is easily characterized. Since $p_{k}(0)=\bar{p}$ for each of the goods with $\phi_{k}(0)>0$, an optimal consumption plan must be of the form (1.4), for some level of total expenditure $c^{j}(0)$. The optimal level of total expenditure must satisfy the Euler condition

$$
u^{\prime}\left(\frac{c^{j}(0)}{\omega^{j}(\phi(0))}\right) \geq \beta(1+i(0)) \frac{\bar{p}}{P^{*}(1)} \Lambda^{j}\left(\tilde{a}^{j}(1) ; \tilde{\boldsymbol{a}}(1)\right),
$$

where

$$
\Lambda^{j}(\tilde{a} ; \tilde{\boldsymbol{a}}(1)) \equiv \frac{\partial V^{j}(\tilde{a} ; \tilde{\boldsymbol{a}}(1))}{\partial \tilde{a}}
$$

measures the marginal utility of additional real wealth at the beginning of period 1 , for units in sector $j$. An optimal plan must satisfy both inequalities (1.12) and (2.27), and at least one of these must hold with equality for each sector $j$.

The following characterization of the function $\Lambda^{j}\left(\tilde{a}^{j}(1) ; \tilde{\boldsymbol{a}}(1)\right)$ is useful for constructing examples of possible equilibria.

Lemma 4. Let the utility functions $u(c ; \bar{\xi}), v(y ; \bar{\xi})$ be given, as well as the coefficients $\left\{\alpha_{k-j}\right\}$. Then we can define a vector function $\boldsymbol{\Lambda}^{*}(\boldsymbol{f})$ for all $\boldsymbol{f} \in U$, such that for each sector $j$, and any vector $\tilde{\boldsymbol{a}}(1)$ consistent with the hypothesis of Lemma 3,

$$
\Lambda^{j}\left(\tilde{a}^{j}(1) ; \tilde{\boldsymbol{a}}(1)\right)=\Lambda^{* j}(\boldsymbol{f}),
$$

where $\boldsymbol{f}$ is the vector of transfers defined by (2.24). The function $\boldsymbol{\Lambda}^{*}(\boldsymbol{f})$ is independent of the value of $\beta$, which matters only through the way in which it enters (2.24). Furthermore,

$$
\lim _{\boldsymbol{f} \rightarrow \mathbf{0}} \boldsymbol{\Lambda}^{*}(\boldsymbol{f})=\boldsymbol{\Lambda}^{*}(\mathbf{0})=u^{\prime}(\bar{y} ; \bar{\xi}) \cdot \boldsymbol{e}
$$

where $\boldsymbol{e}$ is a vector of ones.

This means that the Euler condition (2.27) takes an especially simple form when the vector $\boldsymbol{f}$ defined by (2.24) is small in all of its elements. The next two sections discuss two special cases in which this approximation can be used. 


\subsection{Effective demand failure when liquidity is scarce}

We now consider the equilibrium allocation of resources in the case of an asymmetric disturbance such as a pandemic, under the assumption that policy continues to be of the kind assumed in Proposition 1. While monetary policy responds to shocks under such a regime, it is assumed to respond only to aggregate shocks (determinants of the "natural rate of output" $y_{t}^{*}$ ). Thus (for now) we assume that there is no response of macroeconomic policy to the asymmetric disturbance.

The resulting impairment of effective demand is most dramatic when liquid asset balances are low. In this section, we simplify the analysis by considering the limiting case in which $a(0) \rightarrow 0$. We also continue to assume that $\underline{b}_{j}(0)=0$ for all sectors: there is no credit policy. Note that even in this limiting case, no inefficiency would result as long as only aggregate shocks occur (Proposition 1); thus we can imagine an economy choosing to operate with a very low level of liquid assets, if the ex-ante probability assigned to the occurrence of a pandemic shock has been quite small.

As explained in section 1.1, the fact that $p_{k}(0)=\bar{p}$ for all sectors implies that each sector's spending will be allocated in accordance with (1.4), where however the factors $\left\{\omega_{j}\right\}$ will differ from 1 in the case of an asymmetric disturbance. ${ }^{18}$ It follows that the total demand for the product of any sector $k$ will be given by

$$
y_{k}(0)=\sum_{j=1}^{N} c_{k}^{j}(0)=\sum_{j=1}^{N} A_{k j} \cdot c^{j}(0) .
$$

In vector notation, we can write

$$
\boldsymbol{y}(0)=\boldsymbol{A c}(0),
$$

where $\boldsymbol{y}(0)$ is the $N$-vector indicating the output of each of the $N$ sectors, $\boldsymbol{c}(0)$ is the $N$ vector indicating the total real spending of each of the sectors, and $\boldsymbol{A}$ is the $N \times N$ matrix with element $A_{k j}$ in row $k$ and column $j$.

Clearing of the asset market in period zero requires that in equilibrium, $\sum_{j=1}^{N} b_{j}(0)=$ $a(0)$. It follows that if $a(0) \rightarrow 0$, the only way in which constraint (1.12) can be satisfied for all $j$, with $\underline{b}_{j}(0)=0$ for all sectors, is if $b^{j}(0) \rightarrow 0$ for each sector. Thus in equilibrium, each sector must spend exactly its income, so that $c^{j}(0)=y^{j}(0)$ for each $j$. It then follows from (2.28) that $\boldsymbol{c}(0)=\boldsymbol{A} \boldsymbol{c}(0)$. Thus $\mathbf{c}(0)$ must be a right eigenvector of $\boldsymbol{A}$, with an associated eigenvalue of 1 .

Such an eigenvector must exist. Using the properties of stochastic matrices discussed in Gantmacher (1959, sec. XIII.6), ${ }^{19}$ we can further establish that 1 is the maximal eigenvalue of $\boldsymbol{A}$ (all of its $N-1$ other eigenvalues have modulus less than 1), and that the right eigenvector $\boldsymbol{\pi}$ associated with this maximal eigenvalue is non-negative in all elements. ${ }^{20}$ If we normalize the eigenvector so that $\boldsymbol{e}^{\prime} \boldsymbol{\pi}=1$, then $\boldsymbol{\pi}$ corresponds to the stationary probability distribution of an $N$-state Markov chain for which $\boldsymbol{A}$ defines the transition probabilities.

\footnotetext{
${ }^{18}$ From here onward, we simply write $\omega_{j}$ and $A_{k j}$ for the coefficients defined in (1.5). We omit the argument $\phi(0)$, since we only consider the alternative possible equilibria associated with different policies in the case of a particular asymmetric disturbance $\phi(0)$.

${ }^{19}$ See the proof of Proposition 3 in the appendix.

${ }^{20}$ Of course, the left eigenvector associated with the maximal eigenvalue is $\boldsymbol{e}^{\prime}$, the vector of $1 \mathrm{~s}$.
} 
Since this is the unique right eigenvector with an associated eigenvalue of 1 , equilibrium requires that $\boldsymbol{c}(0)=\Omega \boldsymbol{\pi}$ for some scalar coefficient $\Omega \geq 0$. In order to determine the value of $\Omega$, we recall that intertemporal optimization requires that the Euler condition (2.27) must hold for each sector $j$, and must hold with equality for any sector with $b^{j}(0)>0$. In the limiting case considered in this section, $\boldsymbol{f} \rightarrow \mathbf{0}$, so that Lemma 3 implies that the Euler condition reduces to

$$
u^{\prime}\left(c^{j}(0) / \omega_{j} ; \bar{\xi}\right) \geq u^{\prime}(\bar{y} ; \bar{\xi}) .
$$

Because of the strict concavity of $u(c)$, condition (2.29) can equivalently be written as

$$
c^{j}(0) \leq c^{* j} \equiv \omega_{j} \bar{y} .
$$

The value of $\Omega$ must be small enough for each element of $\boldsymbol{c}(0)$ to be consistent with this upper bound; but at the same time it must be large enough for the inequality to hold with equality for at least one sector. Thus we must have

$$
\frac{1}{\Omega}=\max _{j} \frac{\pi_{j}}{\omega_{j}} \cdot \frac{1}{\bar{y}}>0 .
$$

Note that here the quantities $\left\{\pi_{j}, \omega_{j}\right\}$ depend on the disturbance vector $\phi(0)$; thus we obtain a solution $\Omega=\Omega(\phi(0))$. We can summarize our results as follows.

Proposition 3. Suppose that, despite the occurrence of an asymmetric disturbance $\phi(0)$ in period $t=0$, all policies remain as specified in Proposition 1. And suppose further that, for a given specification of the disturbance vector, we let $a(0) \rightarrow 0$. Then in this limit, the equilibrium level of spending in each sector in period $t=0$ is given by $\boldsymbol{c}(0)=\Omega \boldsymbol{\pi}$, where $\boldsymbol{\pi}$ is the maximal right eigenvector of the matrix $\boldsymbol{A}$ and $\Omega$ is given by (2.31). The equilibrium level of production by each sector is given by $\boldsymbol{y}(0)=\boldsymbol{c}(0)$, and the allocation of each sector's spending across the different goods is given by (1.4).

In all periods $t \geq 1$, the equilibrium allocation of resources continues to be the one specified in Proposition 1. And all equilibrium prices (including the interest rate $i(0)$ ) remain those specified in Proposition 1.

In this solution, there will necessarily be at least one sector (the sector or sectors $j$ for which the maximum value is achieved in the problem on the right-hand side of (2.31)) for which the borrowing constraint does not bind, and as a consequence period zero expenditure $c^{j}(0)$ is at the level $c^{* j}$. But at the same time, in the case of an asymmetric disturbance, there will generally be some sectors that are borrowing-constrained, and hence consume less than this amount. This provides a further reason for the consumption and production of some goods to be inefficiently low — not just lower than in the "normal case", but lower than would be the case in the first-best optimal allocation, taking into account the disturbance — if the shock reduces $\phi_{k}(0)$ in some sectors.

Corollary 1. Suppose that $0 \leq \phi_{k}(0) \leq 1$ for all sectors, and that policy (and the initial level of liquid assets) are as assumed in Proposition 3. Then the quantity $c_{k}^{j}(0)$ of goods of type $k$ consumed in sector $j$ is necessarily no greater than the quantity associated with the first-best optimal resource allocation given the disturbance (characterized in Lemma 2). Furthermore, it will be strictly less than the first-best level (meaning also that the production $y_{k}(0)$ of good $k$ will be inefficiently low), if either (i) sector $j$ is borrowing-constrained, and $\alpha_{k-j}>0$, or (ii) $0<\phi_{k}(0)<1$, and the function $v(y ; \bar{\xi})$ is strictly convex. 
The corollary follows directly from (2.30), which together with (1.4) implies that in the absence of a binding borrowing constraint for sector $j$,

$$
c_{k}^{j}(0)=\alpha_{k-j} \phi_{k}(0) \bar{y}
$$

In the case that $v(y ; \bar{\xi})$ is strictly convex, (1.8) implies that $\phi \bar{y}<y^{*}(\phi)$ for all $0<\phi<1$, so that this will be an inefficiently low level of consumption of good $k$, if $k$ is a good for which $0<\phi_{k}(0)<1$. (Non-borrowing-constrained consumers fail to take into account that the social cost of consuming more of good $k$ is proportional to $v^{\prime}\left(y_{k}(0) ; \bar{\xi}\right)$, which is lower than the value $v^{\prime}(\bar{y} ; \bar{\xi})$ in the "normal case" if $v$ is strictly convex.) Moreover, even if $v$ is linear in $y$ (or $\phi$ is exactly 0 or 1 ), $\phi \bar{y}$ is never larger than $y^{*}(\phi)$. Hence the non-borrowingconstrained level of consumption is less than or equal to the first-best level for each good $k$. At the same time, (2.30) implies that a borrowing constraint can only lower spending relative to the non-borrowing-constrained level. Hence there can be no good for which consumption is too high, under the assumptions of the corollary, and it will be inefficiently low if either the borrowing constraint binds for sector $j$ or $\phi_{k}(0) \bar{y}<y^{*}\left(\phi_{k}(0)\right)$ for sector $k$.

One source of inefficiency in the general case is the fact that prices in period zero are pre-determined at a level that was expected to clear markets in the "normal case," but that no longer correctly reflect supply costs given the disturbance. This is not, however, the only reason why equilibrium is generally inefficient in the case of an asymmetric disturbance. We can set aside the issue of distortions owing to the fact that prices are pre-determined by considering the case of a pandemic shock in which it becomes impossible to safely consume the product of one sector, while the value of consuming other goods is unchanged. We shall (without loss of generality) suppose that sector 1 is the one impacted by the pandemic, and consider a disturbance in which $\phi_{1}(0)=0$, while $\phi_{k}(0)=1$ for all $k \neq 1$. In this special case, the first-best level of production continues to be $\bar{y}$ in all sectors $k \neq 1$, while it falls to zero in sector 1; thus (2.32) coincides with the first-best level of consumption of each good.

Nonetheless, consumption will be inefficiently low in the case of any sector $j$ for which the borrowing constraint binds; and there will necessarily be at least one such sector (sector 1 , which necessarily has no income). And in this case the inefficiency cannot be attributed to the fact that prices are not flexible. The pre-determined prices still imply relative prices for all of the goods that anyone purchases (goods $k \neq 1$ ) that correspond to the marginal rates of substitution in the first-best allocation (and as discussed below, it is possible in this case to achieve the first-best outcome despite the fact that prices are pre-determined, without having to use taxes or subsidies that can effectively change the prices of goods). Nonetheless, equilibrium will be inefficient, under the policies assumed in Proposition 3.

The severity of the inefficiency depends not only on the the disturbance vector $\phi(0)$, but also (crucially) on the network structure of payments. The two cases shown in Figure 1 provide contrasting examples. Figure 2 shows the equilibrium consumption vector $\boldsymbol{c}(0)$ for each of these numerical examples, in the case of the pandemic shock just discussed. In each panel of the figure, the five columns represent total expenditure by units in each of the five sectors. The height of the dashed black border indicates the "normal" level of expenditure (equal to $\bar{y}$ for each sector) - the equilibrium level of expenditure if no pandemic shock occurs, which is also the optimal allocation in that case. The height of the solid red outline for each sector indicates the level $c^{* j}$ that would be optimal given the occurrence of the 


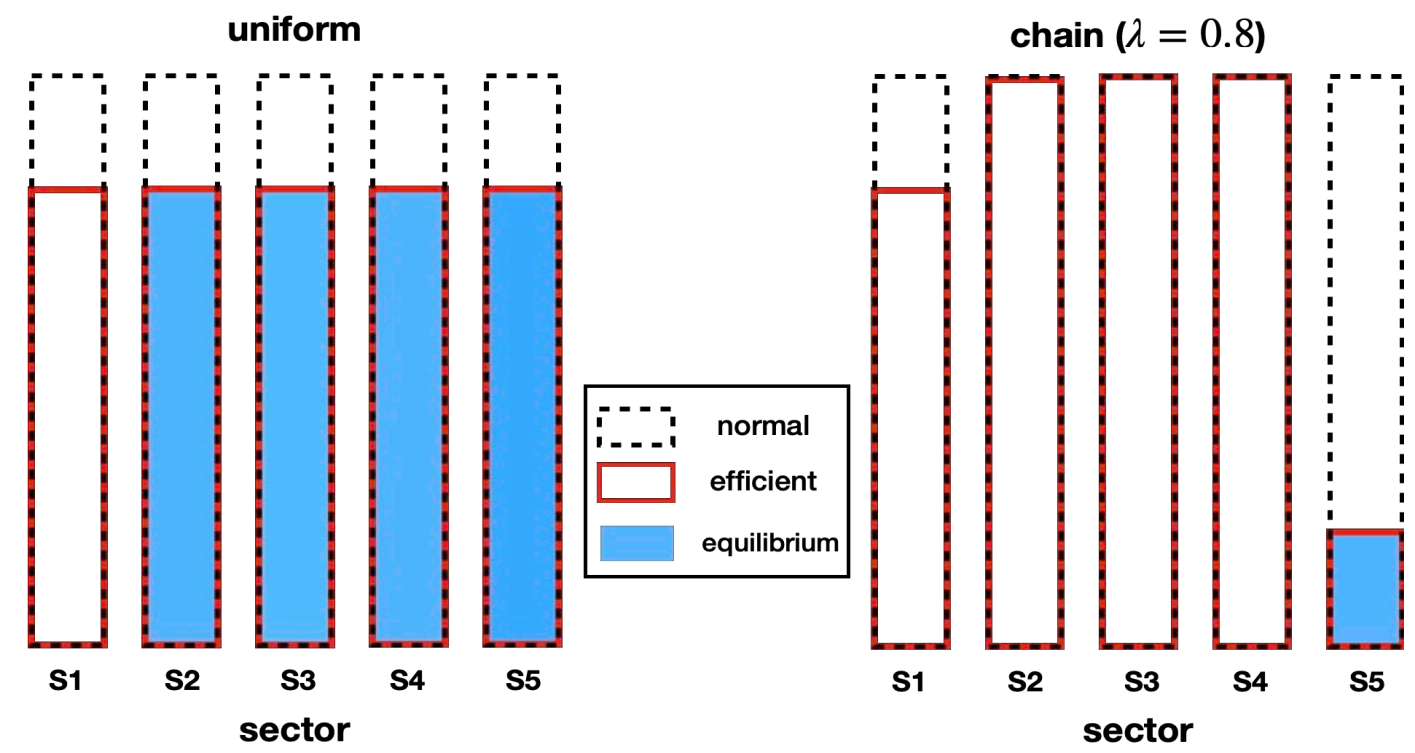

Figure 2: Equilibrium and first-best optimal sectoral expenditure levels in the case of a pandemic shock that requires sector 1 to be shut down, in the case of the two network structures shown in Figure 1.

pandemic shock. (This is necessarily no higher than the normal level and smaller for at least some sectors; thus it is optimal for expenditure and production to decline if a pandemic shock occurs.) The height of the filled blue bar instead indicates the equilibrium level of expenditure $c^{j}(0)$. This is necessarily no higher than the optimal level for any sector, in accordance with Corollary $1 .^{21}$

In the case of a uniform network structure, expenditure collapses completely in sector 1 (which no longer receives any income), but it is reduced in sectors $j \neq 1$ only to the extent that it is efficient for these sectors to reduce their spending (given that they no longer can or should buy sector-1 goods). The collapse of effective demand is much more severe (and the inefficiency much greater) in the case of a "chain" network. Once again, sector 1 cannot spend at all, because it receives no income. But in the case of the "chain" network, this means that sector 2 receives no income other than its own within-sector spending, and the only consistent result is one with zero spending by sector 2 as well. Continuing iteratively in this way, one can show that every sector but sector $N$ must have zero expenditure.

These two cases illustrate two extremes with regard to the degree of collapse of aggregate expenditure and output in the limiting case in which $a(0) \rightarrow 0$. Clearly the network structure makes an important difference. But even in the most benign case (the uniform network of payments), aggregate spending and output are inefficiently low, because spending by sector 1 is inefficiently low. ${ }^{22}$

\footnotetext{
${ }^{21}$ See the appendix, section B.5, for the algebraic calculations used in the figure.

${ }^{22} \mathrm{It}$ is also clear that the empirically relevant network structure is not completely uniform, so that sector 1 will almost certainly not be the only borrowing-constrained sector in the limits as $a(0) \rightarrow 0$. See, for example, Danieli and Olmstead-Rumsey (2020), who document for the US economy that the sectoral composition of the reduction of demand in the COVID-19 crisis was non-uniform in ways that go beyond the simple fact that people in contact-intensive occupations were no longer able to work.
} 


\subsection{Effective demand failure when discounting is minimal}

Another case in which we can use Lemma 4 to simplify the form of the Euler condition, even when there is a severely asymmetric disturbance in period $t=0$, is when $\beta$ is very close to 1 . This might be understood to represent a case in which our discrete "periods" are short in terms of calendar time, so that the asymmetric disturbance with which we are concerned is quite transitory in its effects (though possibly severe while it lasts, as in the case of the disruption of normal patterns of economic activity by COVID-19). Here we fix the single-period utility functions $u$ and $v$, the coefficients $\left\{\alpha_{h}\right\}$ describing the within-period network structure, and the vector $\phi(0)$ specifying the nature of the asymmetric disturbance, but consider the limiting equilibrium as $\beta \rightarrow 1 .^{23}$

Regardless of the values $\left\{b^{j}(0)\right\}$ chosen by units in the various sectors, in the limit as $\beta \rightarrow 1$ we must have $\boldsymbol{f} \rightarrow \mathbf{0}$. Hence by Lemma 4 , the equilibrium allocation and relative prices in periods $t \geq 1$ will involve $y_{j}=\bar{y}, q_{j}^{*}=1$ for all sectors, and the Euler condition will again reduce to the simpler form (2.29). Because in this case we need not have $b^{j}(0)$ near zero for all sectors in order for this simplification to be possible, we can now consider equilibria in which the quantity of liquid assets in period zero need not be negligible, and in which borrowing may be possible as well. This allows us to consider the effects of policies that include transfers in period zero in response to the asymmetric disturbance, or credit policy that relaxes borrowing limits.

Let units in each sector $j$ have initial liquid assets $a^{j}(0) \geq 0$ in period zero (including any fiscal transfers, which may make the asset balances of different sectors unequal), and a borrowing limit $\underline{b}^{j}(0) \leq 0$ (possibly modified by credit policy). In this case, the allocation of each unit's spending across different goods continues to be given by (1.4), so that (2.28) still holds. Because the Euler condition again reduces to (2.29), the upper bound on sectoral expenditure for each sector (i.e., the level of spending if the borrowing limit does not bind) continues to be given by (2.30). However, the level of total spending by any sector $j$ allowed by its borrowing limit is now given by

$$
c^{j}(0) \leq y_{j}(0)+\frac{a^{j}(0)-\underline{b}^{j}(0)}{\bar{p}}=\sum_{k} A_{j k} c^{k}(0)+\frac{a^{j}(0)-\underline{b}^{j}(0)}{\bar{p}} .
$$

where the second term in each of these expressions can now differ from zero, either because $a^{j}(0)$ is a non-negligible amount or because borrowing is possible.

At least one of the inequalities (2.30) and (2.33) must hold with equality for each sector; this implies a consumption function

$$
c^{j}(0)=\min \left\{\frac{a^{j}(0)-\underline{b}^{j}(0)}{\bar{p}}+\sum_{k} A_{j k} c^{k}(0), c^{* j}\right\}
$$

for each sector $j$. The collection of these conditions, one for each $j$, can be written in vector

\footnotetext{
${ }^{23}$ It doesn't matter whether we assume that the numerical value of the inflation target changes as "periods are made shorter." The numerical path of $\left\{\pi^{*}(t)\right\}$ has no consequences for the equilibrium allocation of resources, as long as we continue to assume that $i(0)$ is determined by a policy rule (1.16) that involves that involves the value of $P^{*}(1)$ implied by the target.
} 
form as

$$
\boldsymbol{c}(0)=\min \left\{\frac{1}{\bar{p}} \boldsymbol{\delta}+\boldsymbol{A} \boldsymbol{c}(0), \boldsymbol{c}^{*}\right\},
$$

where min is the operator that maps two $N$-vectors to an $N$-vector, each element of which is the minimum of the corresponding elements of its arguments, ${ }^{24} \boldsymbol{\delta}$ is the $N$-vector with elements $\delta^{j} \equiv a^{j}(0)-\underline{b}^{j}(0)$, and $\boldsymbol{c}^{*}$ is the vector of optimal expenditure levels $\left\{c^{* j}\right\}$. For any vector $\boldsymbol{\delta}$ measuring the tightness of liquidity constraints, equilibrium requires that $\boldsymbol{c}(0)$ be a fixed point of (2.35). This is a multidimensional generalization of the "Keynesian cross" diagram commonly used to explain the derivation of the fiscal multipliers in Keynes (1936, chap. 3). ${ }^{25}$

For any vector $\boldsymbol{\delta}>\boldsymbol{>} \mathbf{0}$, the right-hand side of (2.35) defines a positive concave mapping of the kind for which the results summarized in Cavalcante et al. (2016) allow one to establish that there is a unique fixed point. ${ }^{26}$ Thus equation (2.35) has a unique solution $\boldsymbol{c}(0)=\overline{\boldsymbol{c}}(\boldsymbol{\delta})$. In fact, the piecewise linearity of the mapping allows us to give a closed-form solution to the fixed-point problem, which can also be extended to all vectors $\boldsymbol{\delta} \geq \mathbf{0}$.

Let $C$ be any conjecture about the subset of sectors that are borrowing-constrained (in the sense that $\left.c^{j}(0)<c^{* j}\right)$ in the solution to $(2.35)$; this includes the possibility that no sectors are borrowing-constrained $(C=\emptyset)$. We can restrict the set of conjectures that we need to consider by observing that for vectors $\boldsymbol{\delta}$ near enough to $\mathbf{0}$, the set of unconstrained sectors $U_{0}$ is the set of $j$ for which the maximum value is achieved in the problem on the right-hand side of (2.31). Hence at such points the set of constrained sectors will be $C_{0}$, the complement of $U_{0}$. We can further show that increasing any element of $\boldsymbol{\delta}$ can only reduce the set of sectors that are borrowing-constrained; hence for any $\boldsymbol{a}(0) \geq \mathbf{0}$, the set of constrained sectors $C$ is necessarily an element of $\mathcal{C}$, the set of all subsets of $C_{0}$ (including the empty set).

Now for any conjecture about the subset $C$, we can replace each of the equations in (2.35) by either an equation that states that (2.33) holds with equality (if $j \in C$ ), or an equation that states that $(2.30)$ holds with equality (if $j \neq C$ ). We then obtain a system of linear equations to solve for $\boldsymbol{c}(0)$, that can be written in the form

$$
\boldsymbol{\Phi} \boldsymbol{c}(0)=\boldsymbol{\Psi}_{1} \boldsymbol{\delta}+\boldsymbol{\Psi}_{2} \boldsymbol{c}^{*},
$$

where the coefficients of the matrices depend on the choice of $C$. We can further show the following.

Proposition 4. For any $C \in \mathcal{C}$, the matrix $\boldsymbol{\Phi}$ is invertible, so that the system of equations (2.36) has a unique linear solution,

$$
\boldsymbol{c}^{l o c}(\boldsymbol{\delta} ; C)=\boldsymbol{M} \boldsymbol{\delta}+\boldsymbol{N} \boldsymbol{c}^{*},
$$

where all elements of the matrices $\boldsymbol{M}$ and $\boldsymbol{N}$ are non-negative. Moreover, the fixed-point problem (2.35) has a unique solution, which is just the lower envelope of the finite collection

\footnotetext{
${ }^{24}$ That is, it is the meet of the two vectors, if $\mathbb{R}^{n}$ is treated as a lattice with the partial order $\leq$.

${ }^{25}$ Auclert et al. (2018) similarly propose a multidimensional generalization of the Keynesian cross, but for the allocation of spending across time, rather than across sectors at a given point in time.

${ }^{26}$ See the proof of Proposition 4 in the appendix for details.
} 
of these candidate local solutions:

$$
\overline{\boldsymbol{c}}(\boldsymbol{\delta})=\min _{C \in \mathcal{C}} \boldsymbol{c}^{l o c}(\boldsymbol{\delta} ; C) .
$$

And associated with this unique solution for $\boldsymbol{c}(0)$ is a unique solution for the vector of sectoral output levels $\boldsymbol{y}(0)$, given by (2.28).

We can now consider again the consequences of an asymmetric disturbance in period zero, under the assumption that policy remains as specified in Proposition 1, but now allowing for a non-trivial initial level of liquid assets $a(0)>0$, again divided equally across the $N$ sectors. One conclusion from the previous section that remains unchanged is Corollary 1.

Corollary 2. Suppose again that $0 \leq \phi_{k}(0) \leq 1$ for all sectors, and that all policies remain as specified in Proposition 1, though the initial asset holdings $\left\{a^{j}(0)\right\}$ need not be negligible in size. And for a fixed specification of the intra-period utility functions, the aggregate disturbance vector $\bar{\xi}$, and the asymmetric disturbance vector $\phi(0)$, consider equilibrium in the limit as $\beta \rightarrow 1$. In this limit, the quantity $c_{k}^{j}(0)$ of goods of type $k$ consumed in sector $j$ is necessarily no greater than the quantity associated with the first-best optimal resource allocation given the disturbance (characterized in Lemma 2). Furthermore, it will be strictly less than the first-best level (meaning also that the production $y_{k}(0)$ of good $k$ will be inefficiently low), if either (i) sector $j$ is borrowing-constrained, and $\alpha_{k-j}>0$, or (ii) $0<\phi_{k}(0)<1$, and the function $v(y ; \bar{\xi})$ is strictly convex.

Thus we conclude again that an asymmetric disturbance will generally result in inefficiently low production and consumption of at least some goods, and once again the inefficiency will be greater, the larger the number of sectors that are borrowing-constrained. The existence of a higher level of initial assets $a(0)$ makes no difference (in the $\beta \rightarrow 1$ limit) to the consumption of sectors that are not borrowing-constrained; the only difference is that, for given borrowing limits, a higher level of initial assets makes it less likely that sectors are borrowing-constrained. If the level of initial assets is high enough, no sector will be borrowing-constrained, and in this case, the equilibrium allocation of resources will be the first-best optimal one. How large the required level of initial assets is depends on the degree to which $\phi_{k}(0)$ is reduced in the impacted sectors. (We have shown in Proposition 1 that negligible liquid assets suffice in the case that $\phi_{k}(0)=1$ for all sectors.)

But when $a(0)$ is positive but not extremely large, there can still be inefficiently low production and consumption in period zero in the event of a sufficiently severe asymmetric disturbance. Here we consider again the example of a pandemic shock that requires suspension of all consumption of the product of sector 1, in the case of the two network structures illustrated in Figures 1 and 2. Figure 3 shows how the equilibrium solution for total expenditure $c^{j}(0)$ for each of the sectors varies with the level of total initial liquid assets $a(0)$, under the assumption that initial liquid assets are equally distributed across sectors $\left(a^{j}(0)=a(0) / N\right.$ for each $\left.j\right)$. The two panels present the results for the two different network structures in shown in the corresponding panels of Figure 1. In each panel, the upper envelope plots $c^{a g g}(0) \equiv \sum_{j} c^{j}(0)$ as a function of $a(0)$, and the differently shaded regions decompose aggregate expenditure into the contributions from expenditure by each of the sectors. 
uniform

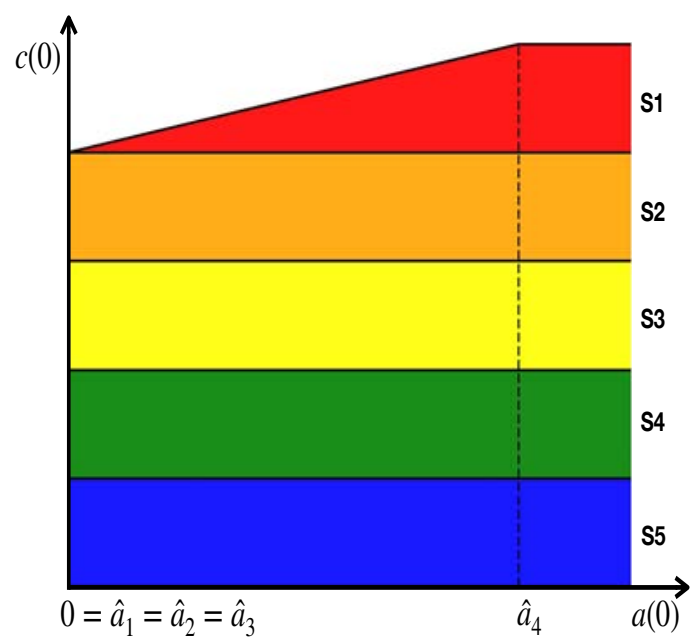

$\operatorname{chain}(\lambda=0.8)$

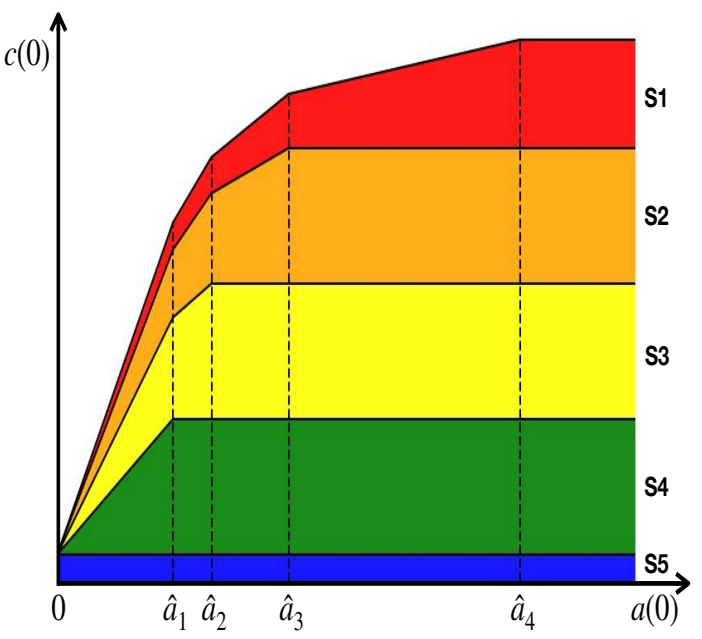

Figure 3: Equilibrium expenditure as a function of total liquid assets $a(0)$ after any transfers in response to the shock, in the case of the two network structures shown in Figure 1. Here liquid assets are assumed to be equally divided among the 5 sectors.

We observe that there is a finite level of initial liquid assets (the level denoted $\hat{a}_{4}$ in both panels), such that $a(0) \geq \hat{a}_{4}$ is a sufficient condition for no sector to be borrowingconstrained. In the case of the kind of asymmetric disturbance considered here, this in turn implies that the equilibrium allocation of resources will coincide with the first-best optimal allocation. ${ }^{27}$ This critical level of initial assets is characterized in the following result.

Corollary 3. Under the assumptions of Corollary 2, the unique equilibrium discussed in Proposition 4 involves no binding borrowing constraint in any sector if and only if

$$
\delta^{j} \geq\left[\sum_{k} \alpha_{k-j} \phi_{k}(0)-\phi_{j}(0)\right] \cdot \bar{p} \bar{y}
$$

for every sector $j$. In this case, the equilibrium allocation of resources in period $t=0$ is given by

$$
c_{k}^{j}(0)=\alpha_{k-j} \phi_{k}(0) \bar{y}, \quad y_{k}(0)=\phi_{k}(0) \bar{y}
$$

for all $j, k$. In the special case that $\underline{b}^{j}(0)=0$ for all $j$ (no credit policy) and $a^{j}(0)=a(0) / N$ for all $j$ (no sectorally-targeted transfers), condition (2.39) holds if and only if the level of initial liquid assets satisfies

$$
a(0) \geq N \bar{p} \bar{y} \cdot \max _{j}\left\{\sum_{k} \alpha_{k-j} \phi_{k}(0)-\phi_{j}(0)\right\} .
$$

\footnotetext{
${ }^{27}$ It is actually not exactly exactly equal to the first-best allocation, but approaches the first-best allocation in the limit as $\beta \rightarrow 1$. Fully achieving the first-best allocation when $\beta<1$ would require fiscal transfers that redistribute income between the sectors; see Proposition 5. But when $\beta \rightarrow 1$, the effect of this redistribution on spending in any individual period becomes negligible.
} 
Corollary 3 shows that the degree of asymmetry of the disturbance in period zero is critical for generating an effective demand failure - that is, a situation in which the inefficiency of the allocation of resources is increased by the consequences of binding borrowing constraints, which reduce the spending both of the constrained sectors themselves and and also of any borrowing-constrained sectors that would otherwise sell more to those sectors. Proposition 1 had already indicated that this is not a problem in the case of disturbances that affect all sectors identically. But we now see that borrowing constraints will also not bind in the case of a sufficiently mild asymmetry in the effects of the disturbance (assuming a non-negligible value for $a(0)$, or at least some possibility of borrowing), since the bounds in (2.39) and (2.41) difference between the value of $\phi_{j}(0)$ for an individual sector and the average value of $\phi_{k}(0)$ for the sectors from which it purchases (weighted by their importance as suppliers for sector $j$ ); this required level of liquidity becomes larger the greater the degree of asymmetry in the elements of the vector $\phi(0)$.

Figure 3 illustrates how the level of initial liquid assets determines which sectors are borrowing-constrained in the event of a pandemic shock. For values of $a(0)$ near zero, the pattern is the one already illustrated in Figure 2, determined by the maximal eigenvector of the matrix $\boldsymbol{A}$. Since a larger quantity of initial liquid assets can only result in fewer borrowing constraints binding, in the case of the uniform network structure (where we have already seen that only sector 1 is constrained, even when $a(0) \rightarrow 0$ ), only sector 1 has a binding borrowing constraint for any $0<a(0)<\hat{a}_{4}$, as shown in the left panel of the figure. In the case of the chain network, instead, for any $0<a(0)<\hat{a}_{1}$, every sector but sector 5 is borrowing-constrained; for any $a_{1} \leq a(0)<\hat{a}_{2}$, all sectors but 4 and 5 are borrowingconstrained; and so on, with progressively more sectors ceasing to be borrowing-constrained as the initial level of liquid assets is increased.

While the inefficiency of the allocation of resources is not as severe as those shown in Figure 2 except in the limiting case of liquid assets near zero, we see that even when liquid assets exist, effective demand failure can result in significant distortions in the event of a sufficiently severe asymmetric disturbance, as in the case of a pandemic. The outcomes shown in Figure 3, however, are still for the case of no policy response to the disturbance. We turn now to the question of how stabilization policies can mitigate the effects of such a shock.

\section{$3 \quad$ Fiscal Transfers and Effective Demand}

We first consider what can be achieved using lump-sum taxes and transfers that are adjusted in response to the occurrence of an asymmetric disturbance. In the case that there are only aggregate disturbances, we have seen (Proposition 1) not only that an optimal allocation of resources can be achieved without any adjustment of fiscal policy in response to shocks, but that (assuming that certain bounds on the path of the public debt are respected) fiscal transfers have no effect on equilibrium prices or quantities. But neither result continues to be true in the case of an asymmetric disturbance that disrupts the circular flow of payments, to a sufficient extent to cause borrowing constraints to bind for some sectors. We now reconsider the role of fiscal transfers as a tool of stabilization policy in this context.

We begin by assuming, as in Proposition 4, that there is no monetary policy response 
to the asymmetric disturbance, meaning that $i(0)=\bar{\imath}$, and that the central-bank reaction function (1.16) for periods $t \geq 1$ continues to be the one appropriate to an environment in which no such shocks occur. The possible role of interest-rate policy (with or without fiscal transfers or credit policy as well) is deferred until the following section.

\subsection{Fiscal policy as "retrospective insurance"}

We first consider whether it is possible to achieve the first-best optimal allocation resources, even in the case of an asymmetric disturbance, through an appropriate policy response. In general, this is not possible, if the instruments of policy are restricted to the ones specified in section 1.3 .

Lemma 5. Suppose that $v(y ; \xi)$ is a strictly convex function of $y$. Then the first-best optimal allocation of resources (characterized in Lemma 2) is not achievable as an equilibrium outcome, under any specification of interest-rate policy, credit policy, or fiscal transfers, except if there is a quantity $\bar{\phi}>0$ such that for every sector $k$, either $\phi_{k}(0)=0$ or $\phi_{k}(0)=\bar{\phi}^{28}$

The reason is that prices are pre-determined at the values $p_{k}(0)=\bar{p}$. This requires a consumption allocation in period zero consistent with (1.4), regardless of the specification of policy; but this is inconsistent with the first-best allocation in Lemma 2, except in two special cases: either $v^{\prime \prime}=0$ over the range of values of $y$ in which the sectoral output levels $\left\{y_{k}(0)\right\}$ all fall (so that Lemma 5 does not apply), or there is only one value of $\phi_{k}(0)$ for all of the sectors with $\phi_{k}(0)>0$.

We can however achieve a first-best outcome in either of these special cases listed in the lemma. In the case that $v\left(y ; \xi_{0}\right)$ is strictly convex, so that Lemma 5 applies, we shall treat the disturbance as a composition of two types of disturbance: an aggregate disturbance that determines a common value of $\bar{\phi}$ for all sectors, and an asymmetric disturbance (a "pandemic shock") that sets $\phi_{k}(0)$ equal to zero (rather than the common level $\bar{\phi}$ ) for one or more sectors $k$ (but not for all of them). When we assume that interest-rate policy in period zero is the one that would be optimal in the absence of an asymmetric disturbance, we allow it to respond to any change that may have occurred in the value of $\bar{\phi}$. The effects of a change in $\bar{\phi}$ for all sectors can furthermore be incorporated into the effects of the aggregate state vector $\xi_{0}$. Thus in treating optimal policy for this case, we can without loss of generality assume (as we have above) that in the case of a pandemic shock, the "normal" value $\phi_{k}(0)=1$ prevails in all sectors except the sector or sectors that are shut down during the pandemic.

Here we also continue, for simplicity, to write our results only for the case in which there are no aggregate disturbances $\left(\xi_{t}=\bar{\xi}\right.$ for all $\left.t \geq 0\right) .{ }^{29}$ Optimal policy in the case of an asymmetric disturbance can then be characterized fairly simply.

\footnotetext{
${ }^{28}$ We may allow cases in which $\phi_{k}(0)=0$ for more than one sector, if we assume weights $\left\{\alpha_{h}\right\}$ such that $\omega_{j}>0$ for every sector $j$ despite this. In this paper, we ensure that $\omega_{j}>0$ by assuming that $\phi_{k}(0)$ can be zero in at most one sector.

${ }^{29}$ Allowing for an aggregate disturbance in period zero, such as a change in the level of $\bar{\phi}$, would require a change in the specification of optimal monetary policy in period zero, of the kind described in Proposition 1 , but would not change the optimal response to an asymmetric disturbance.
} 
Proposition 5. Suppose that there are no aggregate disturbances $\left(\xi_{t}=\bar{\xi}\right.$ for all $\left.t \geq 0\right)$, but that there is an asymmetric disturbance $\phi(0)$ in period zero. Suppose also that either (i) $v\left(y ; \xi_{0}\right)$ is a linear function of $y, v=\nu\left(\xi_{0}\right) \cdot y$; or (ii) for every sector $k$, either $\phi_{k}(0)=0$ or $\phi_{k}(0)=1$. Finally, suppose that policy in periods $t \geq 1$ is consistent with Assumption 1. Then there exists an equilibrium in which the allocation of resources is the first-best optimal allocation defined in Lemma 2, if and only if policy in period $t=0$ is of the following kind: (a) $i(0)$ is determined in the way specified in Proposition 1); and (b) sector-specific lump-sum transfers and taxes are used to ensure that the initial (post-transfer) assets of each sector $j$ are equal to

$$
a^{j}(0)=\frac{a(0)}{N}+\left[\sum_{k} \alpha_{k-j} \phi_{k}(0)-\phi_{j}(0)\right] \cdot \bar{p} \bar{y}
$$

for some $a(0)>0$. (Credit policy is irrelevant: the result holds for any borrowing limits satisfying $\underline{b}^{j}(0) \leq 0$ for all $j$.) In the equilibrium associated with this policy, end-of-period balances are the same for all sectors: $b^{j}(0)=a(0) / N$ for all $j$. As a consequence, borrowing constraints do not bind in any sector, and the allocation of resources in period zero is given by (2.40). Equilibrium prices and quantities in all periods $t \geq 1$ are as specified in Proposition 1.

Among the cases to which this result applies is the kind of pandemic shock considered in Figures 2 and 3.

Note that achievement of the first-best optimum requires no response of monetary policy to the disturbance (in the cases where it can be achieved at all); the optimal interest-rate policy in period zero is unchanged in the absence of an aggregate shock. Nor does it require any use of credit policy; given monetary and fiscal policies of the kind described, credit policy is irrelevant, as borrowing constraints are in any event not binding for any sector. But it is essential that sector-specific fiscal transfers respond appropriately to the realized vector $\phi(0)$, as indicated in (3.1).

It is also noteworthy that condition (3.1) leaves the total quantity of liquid assets $a(0)$ (and hence the size of any government deficit) indeterminate; under an optimal policy, the path of the public debt $\{a(t)\}$ need not respond to the asymmetric disturbance. What the condition does require is redistribution between sectors, depending on the way in which they are impacted by the realization of the asymmetric disturbance.

There is furthermore a simple interpretation for equation (3.1). In the equilibrium that would occur in the absence of any asymmetric disturbance (i.e., if $\phi(k)=1$ for all $k$ ), consumption would be given by $c_{k}^{j}(0)=\alpha_{k-j} \bar{y}$ for all $j, k$. Then if units in each sector would begin period zero with assets $a(0) / N$ in the absence of a disturbance, (3.1) specifies that as a result of the disturbance, each unit receives a transfer in the amount by which spending on its product is reduced after the disturbance,

$$
\bar{p} \bar{y}-\sum_{\ell} \phi_{j} \alpha_{j-\ell} \bar{p} \bar{y}=\left(1-\phi_{j}\right) \bar{p} \bar{y}
$$

and pays a lump-sum tax equal to the amount by which it reduces its spending after the disturbance,

$$
\bar{p} \bar{y}-\sum_{k} \phi_{k} \alpha_{k-j} \bar{p} \bar{y}=\left(1-\sum_{k} \alpha_{k-j} \phi_{k}\right) \bar{p} \bar{y} .
$$


These taxes and transfers balance (so that no change in the size of the public debt is required). They exactly compensate for the disruption to the circular flow of payments that the new pattern of spending would otherwise involve, so that all units end period zero with equal asset balances, and a balanced circular flow of payments continues to be possible in all subsequent periods, as in the equilibrium in Proposition 1, without any further need for sector-specific taxes or transfers. ${ }^{30}$

The optimal fiscal policy in this case amounts to "retrospective insurance" of the kind called for by Milne (2020); ${ }^{31}$ fiscal policy implements the state-contingent transfers that would have been privately agreed upon in (counter-factual) ideal ex-ante contracting. ${ }^{32}$ Note that the case for retrospective insurance made here is independent of the central argument of Milne (2020) or Saez and Zucman (2020), that it is important to prevent business failures, because of the costs involved in re-establishing such businesses once they have failed. While we do not deny that this should also be an important concern, it is not the only reason why a retrospective insurance policy would be efficient (at least if we abstract from the cost of administration of such a policy). Even abstracting from any cost of restarting economic activities in period 1 that did not take place in period zero, as in the present model, the retrospective insurance transfers increase ex-ante welfare.

Note also that while we have referred to implementation of the policy by levying statecontingent lump-sum taxes on the sectors that spend less in the event of a pandemic (because many goods that they normally enjoy are temporarily unavailable) while their incomes are not greatly impacted, the first-best outcome can also be achieved without levying sectorallytargeted taxes on anyone (if one supposes that such taxes are politically unacceptable, while sectorally-targeted transfers are acceptable). Because the value of $a(0)$ in (3.1) is indeterminate (except for the lower bound), it is possible to choose $a(0)$ higher than the quantity of liquid assets that people held prior to the realization of the disturbance, meaning that the government makes net transfers to the private sector (running a deficit in period zero) in response to the disturbance. By choosing a high enough value of $a(0)$, it is possible to ensure that all sectors receive non-negative transfers in period zero. The larger public debt will require correspondingly larger tax collections in later periods; and even though we assume that tax obligations must be the same for all sectors in periods $t \geq 1$, some sectors will end up receiving transfers in period zero greater than the present value of their increased future taxes, while other sectors receive less than that amount. Thus the policy still amounts to a redistribution between sectors, according to how their budgets have been impacted by the asymmetric disturbance, though there need not be sectorally-targeted taxes in any period.

\footnotetext{
${ }^{30}$ Of course, the fact that such taxes and transfers are not needed after $t=0$ depends on our assumption that there are no asymmetric disturbances after $t=0$. In a more general model, similar sectorally-targeted taxes and transfers would be needed each period to compensate for asymmetric disturbances, when they occur.

${ }^{31}$ See also Saez and Zucman (2020) for a similar proposal.

${ }^{32}$ See Woodford (2020) for an explicit analysis of the equilibrium with ex-ante contingent claims contracts, in a generalization of the model presented here. State-contingent transfers as specified in (3.1) would be voluntarily chosen ex ante by all units, owing to the ex-ante rotational symmetry of their situations.
} 


\subsection{The matrix of fiscal transfer multipliers}

Let us now consider more generally what can be achieved by fiscal transfers, that are not necessarily of the precise form specified in (3.1). When $\beta<1$, we will in general have $\boldsymbol{f} \neq \mathbf{0}$, in which case analytical solutions are difficult. We can again obtain simpler conclusions by considering the limit as $\beta \rightarrow 1$, as in section 2.3 .

Rather than there being a single "transfer multiplier," in our model there is an $N \times N$ matrix $\boldsymbol{M}$ of multipliers, the elements of which are

$$
M_{j k} \equiv \bar{p} \cdot \frac{\partial c^{j}(0)}{\partial \delta^{k}}
$$

understood to be right derivatives. ${ }^{33}$ The matrix of multipliers is the same as the matrix $\boldsymbol{M}$ in $(2.37),{ }^{34}$ which we now write more explicitly. For any vector $\boldsymbol{\delta}$, there is a set $C$ of sectors that are borrowing-constrained, in the sense that $c^{j}(0)<c^{* j}$ in the solution (2.38), and a complementary set $U$ (containing at least one sector) that is unconstrained. (Note that $C$ may be the empty set.) If we order the sectors so that all of the sectors in $C$ (if any) come first, then the matrices $\boldsymbol{A}$ and $\boldsymbol{M}$ can be partitioned as

$$
\boldsymbol{A}=\left[\begin{array}{ll}
\boldsymbol{A}_{C C} & \boldsymbol{A}_{C U} \\
\boldsymbol{A}_{U C} & \boldsymbol{A}_{U U}
\end{array}\right], \quad \boldsymbol{M}=\left[\begin{array}{ll}
\boldsymbol{M}_{C C} & \boldsymbol{M}_{C U} \\
\boldsymbol{M}_{U C} & \boldsymbol{M}_{U U}
\end{array}\right],
$$

where submatrix $\boldsymbol{A}_{C C}$ measures the share of spending by each of the constrained sectors on the products of other constrained sectors, and so on. ${ }^{35}$ We can then use this notation to write an explicit expression for the local linear solution (2.37).

Lemma 6. For any specification $C \in \mathcal{C}$ of the set of borrowing-constrained sectors, let the matrix $\boldsymbol{A}$ be partitioned as in (3.2). Then $\boldsymbol{I}-\boldsymbol{A}_{C C}$ is an invertible matrix, and $[\boldsymbol{I}-$ $\left.\boldsymbol{A}_{C C}\right]^{-1}>>0$. It follows that the local linear solution to the system (2.35) can be written in the form (2.37), with matrices of coefficients

$$
\boldsymbol{M}=\left[\begin{array}{cc}
\left(\boldsymbol{I}-\boldsymbol{A}_{C C}\right)^{-1} & \mathbf{0} \\
\mathbf{0} & \mathbf{0}
\end{array}\right], \quad \boldsymbol{N}=\left[\begin{array}{cc}
\mathbf{0} & \left(\boldsymbol{I}-\boldsymbol{A}_{C C}\right)^{-1} \boldsymbol{A}_{C U} \\
\mathbf{0} & \boldsymbol{I}
\end{array}\right],
$$

and all elements of the matrices $\boldsymbol{M}$ and $\boldsymbol{N}$ are non-negative. Moreover, we can equivalently write the non-zero block of $\boldsymbol{M}$ in the form

$$
\boldsymbol{M}_{C C}=\sum_{r=0}^{\infty}\left(\boldsymbol{A}_{C C}\right)^{r}
$$

\footnotetext{
${ }^{33}$ These right derivatives everywhere well-defined, even though the right derivatives can differ from the corresponding left derivatives at values of $\boldsymbol{\delta}$ where the borrowing constraint just ceases to bind for some sector.

${ }^{34}$ Under our definition of $C$, the set $C$ must remain the same in the case of small increases in any of the elements of $\boldsymbol{\delta}$. Hence the solution (2.37) continues to apply, and the matrix $\boldsymbol{M}$ in this solution is the matrix of right derivatives.

${ }^{35}$ Note that the ordering of sectors required for this notation, as well as the partitioning of the rows and columns, depends on the set $C$. Thus $\boldsymbol{A}_{C C}$ has a different meaning for different vectors $\boldsymbol{\delta}$, in the notation used here, even though the matrix $\boldsymbol{A}$ can be given a representation that is independent of $\boldsymbol{\delta}$.
} 
Our model implies that the multiplier effects of fiscal transfers can be quite different, depending both on the sectors receiving the transfer and which sectors' expenditure we are concerned with. One reason why it matters which sectors receive the transfers is that the marginal propensity to consume out of additional income is different for units in different sectors, owing to differences in the degree to which sectors are borrowing-constrained, as stressed by $\mathrm{Oh}$ and Reis (2012). But it also generally matters how transfers are allocated among the different borrowing-constrained sectors, as the size of "higher-round effects" are generally different for different sectors.

Lemma 6 implies that the multipliers are all zero, except those in the block $\boldsymbol{M}_{C C}$, indicating the effects of transfers to borrowing-constrained sectors on the spending by other borrowing-constrained sectors. The solution (3.3) for this sub-matrix expresses the total "multiplier effect" of fiscal transfers as the sum of a "first-round" effect (a unit effect on spending by the sector receiving the transfer), a "second-round" effect (additional spending resulting from the increases in income due to the "first-round" effects), and so on. Note, however, that in our model even the "first-round" effects exist only in the case of transfers to borrowing-constrained units; "second-round" effects exist only to the extent that "firstround" spending increases involve purchases from borrowing-constrained units, and so on. ${ }^{36}$ This implies that in the event of a pandemic shock that suspends consumption of one sector's products, multiplier effects beyond the "first-round" effect can exist only if $N>2{ }^{37}$ hence Guerrieri et al. (2020) find no such effects in their baseline model.

It is possible, in principle, for fiscal transfer multipliers to be sizeable. For example, in the case of the numerical example shown in the right panel of Figure 3, the aggregate expenditure multiplier for a uniformly distributed lump-sum transfer, when pre-transfer asset balances are small enough, ${ }^{38}$ is equal to 2.45 , while the aggregate expenditure multiplier for transfers targeted to units in sector 1 only would equal 4.75. But the multipliers depend not only on how the transfers are targeted, but on the network structure of payments. In the case of the uniform network structure shown in the left panel of Figure 1, the aggregate expenditure multiplier for a uniformly distributed lump-sum transfer is only 0.20 , even when initial asset balances are extremely small.

Figure 3 illustrates a general principle: transfer multipliers are largest when existing liquid asset balances are low (and borrowing constraints are tight). As the size of the transfers is increased, the multiplier effect of further transfers will generally decline, as additional sectors cease to be borrowing-constrained. The following observation is a direct consequence of Proposition 4.

Corollary 4. Suppose that all policies remain as specified in Proposition 1, except that

\footnotetext{
${ }^{36}$ The conclusion that the multiplier is zero in the case of transfers to sectors that are not borrowingconstrained depends on the simplification of considering the limiting case in which $\beta \rightarrow 1$. When $\beta<1$, there is instead a small effect on the current spending of unconstrained units of receiving greater-thanaverage transfers, so that their intertemporal budget increases despite the anticipation of higher future taxes. Nonetheless, the effects on current spending by such units remain small, under realistic assumptions about the discount factor, because of their desire to smooth expenditure over a long horizon.

${ }^{37}$ If $N=2$, the set of constrained sectors $C$ must consist only of sector 1 , as there must be at least one unconstrained sector. And $A_{11}=0$, since no one can purchase sector- 1 goods during the pandemic. Hence $A_{C C}=0$, and all terms corresponding to $r \geq 1$ in (3.3) vanish.

${ }^{38}$ The calculations are explained in the appendix, section C.4.
} 
(possibly sector-specific) lump-sum transfers occur at the beginning of period zero, in response to the asymmetric disturbance. Let post-transfer initial asset holdings be

$$
a^{j}(0)=\alpha^{j}+\gamma^{j} \cdot s
$$

for each sector $j$, where the quantities $\alpha^{j} \geq 0$, with $\sum_{j} \alpha^{j}>0$, denote each sector's pretransfer asset holdings; the coefficients $\gamma^{j} \geq 0$, with $\sum_{j} \gamma^{j}=1$, indicate the share of transfers going to units in each sector $j$; and $s \geq 0$ indicates the total size of the transfer. For this one-parameter family of possible transfer policies, let

$$
m_{j}(s) \equiv \bar{p} \cdot \frac{\partial c^{j}(0)}{\partial s}
$$

(where again we mean a right derivative) measure the multiplier effect of additional transfers on spending by sector $j$, for any possible scale of transfers $s$. Then for each sector $j, m_{j}(s)$ is a piecewise constant, non-increasing function of $s$, that falls to zero for all $s \geq \bar{s}$, where $\bar{s}$ is finite.

Given a matrix $\boldsymbol{M}$ of multiplier effects on sectoral expenditure levels, the matrix $\boldsymbol{M}^{Y} \equiv$ $\boldsymbol{A} \boldsymbol{M}$ then indicates the multiplier effects of transfers to each of the sectors on economic activity (output) of each of the sectors. Since the elements of $\boldsymbol{M}$ are all non-negative, the output multipliers $\boldsymbol{M}^{Y}$ are all non-negative as well. And it follows from Corollary 4 that the output multipliers must decrease as the size of transfers is increased, falling to zero in the case of large enough transfers.

\subsection{Transfer policy and welfare}

We have shown that the multiplier effect of lump-sum transfers on economic activity in each of the different sectors is necessarily non-negative. But a more reasonable goal of policy, of course, should be not to increase economic activity as an end in itself, but rather to increase welfare. Here we consider the effect of lump-sum transfers on the ex-ante welfare measure derived in Lemma 1.

We first note that the ex-ante welfare measure can equivalently be chosen to be

$$
\sum_{j=1}^{n} U^{j}(0)+\frac{\beta}{1-\beta} \sum_{j=1}^{N}\left[\bar{U}^{j}-U^{*}\right]
$$

where $\bar{U}^{j}$ is the stationary level of the utility flow (1.2) associated with the stationary allocation for all $t \geq 1$ characterized in Lemma 3 , and $U^{*}$ is the value of that stationary utility flow (the same for all $j$ ) in the first-best allocation of resources for periods $t \geq 1$. For a given specification of preferences, this differs from (1.7) only by a constant, which does not affect the welfare ranking of alternative possible allocations of resources; but it has the advantage over (1.7) of being a measure with a well-behaved limit as $\beta \rightarrow 1$.

Lemma 7. Suppose that policy in all periods $t \geq 1$ is consistent with Assumption 1, and that we specify policy in period zero by values $a^{j}(0) \geq 0$ such that $\sum_{j} a^{j}(0)>0$, values $b^{j}(0) \leq 0$, 
and a parameter $\psi \equiv \beta(1+i(0)) \bar{p} / P^{*}(1)>0$ which measures the discrepancy between the real rate of interest and the "natural rate" defined in (1.17). Let us consider different values for the discount factor $\beta$, while holding fixed all other aspects of preferences, and the policy parameters just listed; and for all values of $\beta$ close enough to 1 , let the equilibrium for periods $t \geq 1$ be the stationary equilibrium characterized in Lemma 3. Then as $\beta$ approaches 1, we have

$$
\lim _{\beta \rightarrow 1} \frac{\beta}{1-\beta} \sum_{j=1}^{N}\left[\bar{U}^{j}-U^{*}\right]=0
$$

for any policy. Hence one policy implies a higher value for (3.5) in the limit as $\beta \rightarrow 1$ if and only if it implies a higher value for

$$
W_{0} \equiv \sum_{j=1}^{N} U^{j}(0)
$$

It follows that in evaluating welfare under alternative policies in the limiting case in which $\beta \rightarrow 1$, we need consider only the effects on the policy on $W_{0}$, which involves only the allocation of resources in period zero.

We can then use our conclusions about the multiplier effects of lump-sum transfers to calculate effects of such transfers on the welfare measure $W_{0}$. For each sector $j$, let $w_{j} \equiv$ $\bar{p} \cdot \partial W_{0} / \partial a^{j}(0)$ be the effect on welfare per unit transfer to sector $j$, for fixed values of the other policy parameters (in the limit as $\beta \rightarrow 1$ ); and let $\boldsymbol{w}$ be the vector with these components (i.e., the welfare gradient). Then differentiation of (3.7) implies that

$$
\boldsymbol{w}^{\prime}=\left[\boldsymbol{g}^{\prime}+\boldsymbol{h}^{\prime} \boldsymbol{A}\right] \boldsymbol{M}
$$

where $\boldsymbol{g}$ and $\boldsymbol{h}$ are the vectors with elements

$$
g_{j} \equiv u^{\prime}\left(c^{j}(0) / \omega_{j} ; \bar{\xi}\right)-u^{\prime}(\bar{y} ; \bar{\xi}), \quad h_{j} \equiv v^{\prime}(\bar{y} ; \bar{\xi})-v^{\prime}\left(y_{j}(0) ; \bar{\xi}\right)
$$

We further observe that $g_{j}>0$ whenever $c^{j}(0)<c^{* j} \equiv \omega_{j} \bar{y}$, and that $h_{j} \geq 0$ whenever $y_{j}(0)<\bar{y}$. This allows us to sign the elements of the welfare gradient under fairly general conditions.

Proposition 6. Suppose that $0 \leq \phi_{k}(0) \leq 1$ for all sectors, and consider the effects of small additional lump-sum transfers, starting from a situation in which policy is specified as in Lemma 7, with $\psi=1$ (i.e., monetary policy as in Proposition 1). Then $\boldsymbol{w} \geq \mathbf{0}$, so that a lump-sum transfer cannot reduce welfare (in the $\beta \rightarrow 1$ limit), no matter how large the transfer may be, and no matter how it is distributed across sectors. Moreover, $w_{j}>0$ (so that a transfer to sector $j$ increases welfare) if and only if sector $j$ is borrowing-constrained in the absence of the additional transfer (i.e., if $j \in C$ ), while $w_{j}=0$ if the sector is unconstrained.

Thus not only is it possible for lump-sum transfers to increase welfare; in the $\beta \rightarrow 1$ limit, they necessarily increase welfare, as long as at least some of the transfers go to sectors that would be borrowing-constrained in the absence of the transfers. Thus careful targeting of transfers is not necessary in order for them to increase welfare. Moreover, in the case that 
it is possible to achieve the first-best optimal allocation with some combination of policies (i.e., when this is not precluded by Lemma 5), in the $\beta \rightarrow 1$ limit this no longer requires the carefully targeted transfers specified in Proposition 5. Instead, the first-best outcome can be achieved by large enough lump-sum transfers, almost independently of how they are distributed.

Proposition 7. Suppose again that the assumptions of Proposition 5 are satisfied. Then in the limit as $\beta \rightarrow 1$, the equilibrium allocation of resources approaches the first-best optimal allocation defined in Lemma 2, if policy in period $t=0$ is of the following kind: (a) $i(0)$ is determined in the way specified in Proposition 1; and (b) lump-sum transfers ensure that (2.39) holds for every sector $j$. In this limiting equilibrium, borrowing constraints do not bind in any sector, and the allocation of resources in period zero is given by (2.40). Equilibrium prices and quantities in all periods $t \geq 1$ are as specified in Proposition 1.

Note that condition (2.39) is satisfied by almost any distribution of lump-sum transfers, as long as they are large enough. In the case of a transfer policy of the form (3.4), the condition is satisfied for all large enough values of $s$, as long as $\gamma^{j}>0$ for each of the sectors with $\phi_{j}(0)<\sum_{k} \alpha_{k-j} \phi_{k}(0) .{ }^{39}$ Thus transfers need not be sectorally-targeted at all; in the case of uniform transfers (so that $a^{j}(0)=a(0) / N$ for all $j$ ), the condition is satisfied, and the first-best optimal allocation will be achieved, as long as $a(0)$ is large enough. ${ }^{40}$

When $\beta<1$, a more specific pattern of transfers is required in order to achieve the first-best outcome, as indicated in Proposition 5, because the transfers must adjust sectoral budgets so that (i) borrowing constraints no longer bind in period zero for any sector, and (ii) all sectors end period zero with identical end-of-period balances. But in the limit as $\beta \rightarrow 1$, condition (ii) ceases to matter, as differences in the assets carried into period one by different sectors have only a negligible effect on per-period spending in each of the periods $t \geq 1$; the marginal utility of income remains essentially the same for all sectors, as indicated by Lemma 4. In this case, it is important that transfers be large enough to prevent borrowing constraints from binding in any sector, but no significant distortions result from "unnecessary" transfers to some sectors.

Note also that Proposition 7 implies that if $v(y ; \bar{\xi})$ is a linear function of $y$, it is possible to obtain the first-best allocation of resources despite the occurrence of an asymmetric disturbance, even when policy is of the kind specified in Proposition 1, so that policy does not respond to the asymmetric disturbance at all. This result obtains if the initial level of liquid assets $a(0)$ satisfies (2.41) even in the absence of any fiscal transfers in response to the shock. For a given level $a(0)>0$, the condition is satisfied as long as the sectoral nonuniformity of the disturbance $\phi(0)$ is not too great. Our result here recalls Leijonhufvud's (1973) concept of a "corridor" within which market mechanisms can be relied upon to be self-stabilizing, so that Keynesian policies are needed to restore proper functioning only in the case of disturbances large enough to move the economy outside the "corridor." Our account of the nature of effective demand failures is in many ways similar to Leijonhufvud's, but it implies that "the corridor" should be defined not as a state in which shocks to the

\footnotetext{
${ }^{39}$ In the case of a pandemic shock that shuts down one sector without affecting the others, this condition requires only that some of the transfers go to the sector directly impacted by the shock.

${ }^{40}$ In the case of no credit policy $\left(\underline{b}^{j}(0)=0\right.$ for all $\left.j\right)$, the required level of $a(0)$ is given by $(2.41)$.
} 
economy are sufficiently small, but rather as one in which they are sufficiently symmetric in their effects on the income and spending of different parts of the economy.

\subsection{Credit policy as stabilization policy}

Credit policy can also be a useful response to disruption of the circular flow of payments by an asymmetric disturbance. Indeed, in our model, relaxation of sectoral borrowing limits (by allowing government-guaranteed borrowing up to a certain amount) has effects that are similar to the effects of transfers to units in that sector. ${ }^{41}$

In particular, in our model a uniform relaxation of the period-zero borrowing limit in each sector by a common amount $\Delta$ (reducing $\underline{b}^{j}(0)$ by $\Delta$ for each $j$ ) has an identical effect as a uniform lump-sum transfer of $\Delta$ (increasing $a^{j}(0)$ by $\Delta$ for each $j$ ). In either case, the amount that each unit can spend in period zero without violating their borrowing constraint is increased by $\Delta$; and the present value of their future obligations (future lump-sum taxes in the case of the transfer policy, debt repayments in the case of credit policy) is increased by exactly $\Delta$ as well. Since we have shown that sufficiently large uniform transfers can achieve the first-best outcome in the $\beta \rightarrow 1$ limit (Proposition 7 ), it follows that a sufficiently large uniform relaxation of borrowing limits can achieve the first-best outcome in this limiting case as well.

In the case that the degree of relaxation of borrowing limits differs across sectors, the effects of credit policy are no longer identical to those of similarly distributed transfers, because the additional borrowing allowed owing to credit policy must be repaid by the units that increase their borrowing, while transfers increase the future tax obligations of all units uniformly even when the transfers are not uniformly distributed. However, in the limit as $\beta \rightarrow 1$, this difference in the distribution of repayment obligations in periods $t \geq 1$ has only a negligible effect on the allocation of resources. Hence in this limit, the effects of a policy that reduces $\underline{b}^{j}(0)$ by an amount $\Delta^{j}$ (that may differ across sectors) are identical to those of a lump-sum transfer of $\Delta^{j}$ to units in sector $j$ at the beginning of period zero. This can be seen from the fact that equations (2.35) and the solution (2.38) involve only the vector $\boldsymbol{\delta}$, which is affected in the same way by sectorally-targeted transfers and sector-specific credit policy. Thus the matrix of multipliers characterized in Lemma 6 applies equally to the effects of sector-specific credit policy on expenditure in the different sectors.

There are nonetheless respects in which credit policy and transfer policy should not be regarded as perfect substitutes for one another. First, when $\beta<1$, the differing effects on repayment obligations in periods $t \geq 1$ do matter. Hence in Proposition 5, we are able to specify sectorally-targeted transfers that are necessary in order to achieve the first-best outcome, regardless of what is assumed about credit policy; it would not be possible to achieve the first-best outcome (except in the limit as $\beta \rightarrow 1$ ) using credit policy alone. This is an advantage of fiscal transfers over credit policy as a tool of stabilization policy. At the same time, there is an advantage of credit policy as well. Our model assumes that government debt can be repaid through revenues raised by lump-sum taxes, but in practice,

\footnotetext{
${ }^{41}$ Bigio et al. (2020) also compare credit policy with fiscal transfers, in a more developed model of how borrowing constraints are determined. Araújo and Costa (2021) similarly discuss how a modification of bankruptcy law in response to a pandemic shock, or endogenous renegotiation of private loan contracts, can substitute for fiscal transfers in improving stabilization.
} 
distorting taxes may be the only source of revenue. If so, credit policy has the advantage of not creating the distortions that would result from an increase in future taxation of labor income (for example) in order to finance the transfers. This is an advantage of using credit policy, at least to some extent, if the associated administrative costs are not too great.

\section{The Role of Interest-Rate Policy}

In the previous section, we have considered the effects of fiscal transfers and credit policy, under the assumption that interest-rate policy does not respond at all to the asymmetric disturbance. We now consider what can be achieved by a response of interest-rate policy to such a disturbance. Propositions 5 and 7 have shown that under certain conditions, fiscal transfers can achieve the first-best allocation of resources without any need for a change in monetary policy. But what if fiscal policy does not respond, or does not respond ideally, owing to political or administrative constraints? To what extent can monetary policy be used instead?

In the equilibria shown in Figure 3 for cases in which the initial level of liquid assets satisfies $a(0)<\hat{a}_{4}$, equilibrium output is below the efficient level (given the disturbance to fundamentals) in some or all of the sectors not directly affected by the pandemic shock. It is not inefficiently low in the impacted sector, sector 1 , since it is optimal for production to cease temporarily in that sector; but there are no sectors in which activity is inefficiently high, in the absence of a policy response.

Given Proposition 2, it might seem natural to suppose that the central bank's interestrate target should be cut in response to such a real disturbance. And our model is one in which equilibrium activity in period zero can be increased or decreased by interest-rate policy; thus to the extent that one thinks about stabilization policy in terms of an aggregate output gap, it should be possible to eliminate the gap entirely by a sufficiently large cut in interest rates, assuming that the effective interest-rate lower bound does not preclude this. Thus it is often supposed that if counter-cyclical fiscal policy is also needed, this is only because the lower bound may not allow interest rates to be reduced to the degree needed in the case of a severe disturbance. And in fact our model is one in which the zero lower bound never constrains how much it should be possible to reduce the real interest rate (which is what matters for aggregate demand), if the central bank is willing to commit itself to more inflationary policy in the future.

Nonetheless, monetary policy remains a decidedly second-best policy instrument for dealing with the inefficiencies created by an effective demand failure resulting from a pandemic shock. The problem is not that interest-rate policy cannot increase economic activity in these circumstances - the elasticity of aggregate output with respect to changes in the real interest rate is determined by the intertemporal elasticity of substitution, in the same way as in the case of the response to aggregate disturbances considered in Proposition 2. It is rather that the composition of the added expenditure that can be stimulated by interest-rate cuts will necessarily be inefficient, and (depending on the network structure of payments) may be severely so. 


\subsection{Interest-rate policy when liquidity is negligible}

Let us first consider how the analysis in section 2.2 is changed if we suppose that the central bank cuts $i(0)$ in response to the asymmetric disturbance. It continues to be the case that if $a(0)$ is small and $\underline{b}^{j}(0)=0$ for all $j$, the equilibrium from period $t=1$ onward must be one with $\boldsymbol{f}$ near $\mathbf{0}$. However, (2.29) now takes the more general form

$$
u^{\prime}\left(c^{j}(0) / \omega_{j} ; \bar{\xi}\right) \geq \psi u^{\prime}(\bar{y} ; \bar{\xi}),
$$

where $\psi>0$ is the coefficient defined in Lemma 7, measuring the degree to which $i(0)$ differs from the "normal" policy assumed in Proposition 1. (A value $\psi<1$ means that the interestrate target is reduced in response to the asymmetric disturbance.) This in turn implies that (2.30) takes the more general form

$$
c^{j}(0) \leq \hat{c}^{j}(\psi) \equiv \omega_{j} \hat{y}(\psi)
$$

generalizing (2.30), where $\hat{y}(\psi)$ is the quantity implicitly defined by

$$
u^{\prime}(\hat{y}(\psi) ; \bar{\xi})=\psi u^{\prime}(\bar{y} ; \bar{\xi})
$$

Because $u(c ; \bar{\xi})$ is strictly concave, $\hat{y}(\psi)$ is a monotonically decreasing function.

We then obtain the following generalization of Proposition 3.

Proposition 8. Suppose that, despite the occurrence of an asymmetric disturbance $\phi(0)$ in period $t=0$, all policies remain as specified in Proposition 1, except that $i(0)$ responds to the shock. And suppose further that, for a given specification of the disturbance vector and the policy response $\psi$, we let $a(0) \rightarrow 0$. Then in this limit, the equilibrium level of spending in each sector in period $t=0$ is given by $\boldsymbol{c}(0)=\Omega \boldsymbol{\pi}$, where $\boldsymbol{\pi}$ is the maximal right eigenvector of the matrix $\boldsymbol{A}$ and $\Omega$ is given by

$$
\frac{1}{\Omega}=\max _{j} \frac{\pi_{j}}{\omega_{j}} \cdot \frac{1}{\hat{y}(\psi)}>0,
$$

where $\hat{y}(\psi)$ is defined in (4.3). The equilibrium level of production by each sector is given by $\boldsymbol{y}(0)=\boldsymbol{c}(0)$, and the allocation of each sector's spending across the different goods is given by (1.4).

In all periods $t \geq 1$, the equilibrium allocation of resources continues to be the one specified in Proposition 1. And all equilibrium prices (except the interest rate $i(0)$ ) remain those specified in Proposition 1.

Note that (4.4) reduces to (2.31) under our previous assumption that $\psi=1$.

It follows that our model implies that $c^{a g g}(0)$, and correspondingly aggregate output $y^{a g g}(0)$, increases in proportion to $\hat{y}(\psi)$ if the interest-rate target is changed. This is the same interest-rate elasticity of aggregate output as exists in the case that only aggregate disturbances exist: in that case, $c^{j}(0)=y_{j}(0)=\hat{y}(\psi)$ for every sector, so that also in that case $y^{a g g}(0)$ grows in proportion to $\hat{y}(\psi)$. Thus the fact that borrowing constraints may bind for many sectors need not imply any lower interest-elasticity of output in the case of an effective demand failure. And the Inada conditions assumed for the function $u(c ; \bar{\xi})$ imply 
that $\hat{y}$ can be driven arbitrarily close to zero by raising the real interest rate enough, and made arbitrarily large by lowering the real interest rate enough; ${ }^{42}$ thus the model implies that a very great degree of control over aggregate output (in the short run) is possible using monetary policy, even during the crisis created by a pandemic shock.

Nonetheless, monetary policy is not well-suited to correct the distortions created by a pandemic shock. While lowering interest rates should increase spending and hence output, the sectoral composition of the spending and output that are stimulated need not correspond to the kinds are most needed in order to increase welfare. In fact, an interest-rate cut need not increase welfare at all, as shown by the following (admittedly special) example.

Corollary 5. Let the assumptions of Proposition 8 be satisfied. In addition, suppose that $v(y ; \bar{\xi})=\nu \cdot y$ for some $\nu>0$ (that may depend on $\bar{\xi}$ ), and that the disturbance $\phi(0)$ is such that the sectors that are borrowing-constrained in period zero are unable to consume at all. Then $W_{0}$ is maximized when $\psi=1$; that is, if there is no response of monetary policy to the disturbance.

The cases shown in Figure 2 provide two examples in which the assumption in the corollary about the equilibrium pattern of consumption (which depends on the eigenvector $\boldsymbol{\pi}$ ) is satisfied. In such a case, there is no spending by borrowing-constrained sectors, and no production by those sectors either. A reduction of $i(0)$ increases spending only in the unconstrained sectors, and only increases production in those same sectors. Hence only types of consumption $c_{k}^{j}(0)$ increase that are already at the efficient level when $\psi=1$; further increases in this kind of spending increases ex ante utility by an amount less than the increase in the disutility of supplying the goods, and ex-ante welfare is necessarily decreased. Thus while the allocations of resources depicted in Figure 2 are far from the first-best optimal allocation (especially in the case shown in the right panel), welfare cannot be improved in either of these cases by cutting interest rates.

\subsection{Interest-rate policy when discounting is minimal}

Let us next consider how the analysis in section 2.3 is modified if interest-rate policy responds to the asymmetric disturbance. In the limit as $\beta \rightarrow 1$, we again must have $\boldsymbol{f} \rightarrow \boldsymbol{0}$, regardless of the policy chosen in period zero. The Euler condition again reduces to (4.1), which again implies (4.2). The derivation of the consumption function (2.34) then proceeds as before, except that we must replace the constant $c^{* j}$ by the upper bound in (4.2), that depends on $\psi$. The system of equations that determine the sectoral expenditure levels then takes the form

$$
\boldsymbol{c}(0)=\min \left\{\frac{1}{\bar{p}} \boldsymbol{\delta}+\boldsymbol{A c}(0), \boldsymbol{\omega} \cdot \hat{y}(\psi)\right\}
$$

generalizing (2.35), where $\boldsymbol{\omega}$ is the vector with $j$ th element equal to $\omega_{j}$.

For a given specification of policy $(\boldsymbol{\delta}, \psi)$, we can show that the "multivariate Keynesian cross" system (4.5) has a unique fixed point. This allows the following characterization of equilibrium under an arbitrary monetary policy.

\footnotetext{
${ }^{42}$ Because of the zero lower bound on the nominal interest rate, of course, very low real interest rates can be achieved only by creating an expectation of high inflation.
} 
Proposition 9. Suppose that policy in all periods is consistent with Assumption 1, and that policy in period zero is specified as in Lemma 7 . Then in the limit as $\beta \rightarrow 1$, there is a unique equilibrium in which both prices and the allocation of resources in all periods $t \geq 1$ are the ones specified in Proposition 1. In period zero, the vector of sectoral expenditure levels is

$$
\boldsymbol{c}(0)=\boldsymbol{c}(\boldsymbol{\delta} ; \psi)=\frac{\hat{y}(\psi)}{\bar{y}} \cdot \overline{\boldsymbol{c}}\left(\frac{\bar{y}}{\hat{y}(\psi)} \boldsymbol{\delta}\right),
$$

where $\overline{\boldsymbol{c}}(\boldsymbol{\delta})$ is the solution to (2.35) given in (2.38). The pattern of spending on individual goods is then given by (1.4), and the sectoral levels of production are given by (1.14).

Given this solution, it is straightforward to compute the effects of an interest-rate reduction on the allocation of resources in period zero, given a fixed specification of both lump-sum transfers and credit policy. Aggregate output is necessarily increased, but not all types of consumption are increased equally, and the additional increases in spending become more narrowly concentrated as the degree to which the real interest rate is cut is made deeper.

Corollary 6. Consider how the period zero allocation in Proposition 9 varies with $\psi$, for fixed policy parameters $\boldsymbol{\delta} \geq \mathbf{0}$. As $\psi$ is decreased, $y^{\text {agg }}(0)$ increases; moreover, $y^{\text {agg }}(0)$ is a piecewise linear, concave function of $\hat{y}(\psi)$. Similarly, each of the components of this aggregate, $c_{k}^{j}(0)$ for each $j, k$, and $y_{k}(0)$ for each $k$, is a non-decreasing, piecewise linear, concave function of $\hat{y}(\psi)$. As $\psi$ is reduced (and $\hat{y}(\psi)$ increases), the set $C$ of borrowing-constrained sectors is non-decreasing. And there exists a $\underline{\psi}>0$ such that for any $\psi<\underline{\psi}$, the set of borrowingconstrained sectors is equal to $C_{0}$, the set of constrained sectors in the case of negligible liquidity, identified in Proposition 3 (and the maximal element of $\mathcal{C}$ ). For any $\psi$ in this range, the equilibrium vector of sectoral expenditure levels will be

$$
\boldsymbol{c}(0)=\left[\begin{array}{c}
\boldsymbol{M}_{C C} \\
\mathbf{0}
\end{array}\right] \frac{\boldsymbol{\delta}_{C}}{\bar{p}}+\frac{\boldsymbol{\pi}}{\max _{\ell}\left(\pi_{\ell} / \omega_{\ell}\right)} \hat{y}(\psi),
$$

where $\boldsymbol{\pi}$ is again the eigenvector referred to in Proposition 3, and we partition both $\boldsymbol{M}$ and $\boldsymbol{\delta}$ according to the sectors belonging to $C_{0}$ and $U_{0}$ respectively.

Thus for all low enough real interest rates, the solution for $\boldsymbol{c}(0)$ (and hence the solutions for consumption and production of all goods) is the sum of two terms: a term that is proportional to $\hat{y}(\psi)$, identical to the solution in Proposition 8 for the case of negligible liquidity, plus a term proportional to the elements of $\boldsymbol{\delta}_{C}$, that is independent of interestrate policy. In this low-interest-rate case, the only marginal effects of further interest-rate cuts will be increases in expenditure (and similarly production) by the different sectors in proportion to the elements of the eigenvector $\boldsymbol{\pi}$, just as in Proposition 8.

The conclusion that progressively larger increases in $\hat{y}(\psi)$ have diminishing marginal effects might seem parallel to our conclusion in Corollary 4 that the multiplier effects of fiscal transfers diminish as the scale of the transfers is increased. However, the reason for the diminishing effects on economic activity of larger interventions is quite different in the two cases. In the case of fiscal transfers, larger transfers progressively reduce the number of sectors that continue to be borrowing-constrained; additional transfers then have less effect, because there is less of a problem of effective demand failure for them to solve. In the case 
of interest-rate cuts, instead, larger reductions in the real interest rate progressively increase the number of sectors that are borrowing-constrained, so that interest-rate policy has fewer and fewer channels through which it can increase spending. The difference is important: in the case of large transfers, while the effect on aggregate spending diminishes, the remaining effects are concentrated on the few remaining sectors that are still financially constrained; but in the case of large interest-rate cuts, the remaining effects are concentrated on the few sectors that are not financially constrained.

This matters for the welfare effects of policy. In the case of fiscal transfers, we have shown that (at least under the conditions assumed in Proposition 6) additional fiscal transfers can only increase ex-ante welfare, no matter how large they are, and now matter how they are targeted; and under nearly any possible distribution of the transfers, sufficiently large transfers will achieve the first-best allocation (Proposition 7). Instead, even in cases where a moderate reduction of the real interest rate will increase welfare, further interest-rate cuts will instead begin to reduce welfare. And the point at which interest-rate cuts become counterproductive will generally be one in which economic activity remains inefficiently low in some sectors; the continued existence of under-employed resources need not imply that further interest-rate reductions are desirable.

Indeed, it need not be desirable to cut interest rates at all, in response to an asymmetric disturbance, and despite a fiscal response that is inadequate to fully counteract the shock.

Corollary 7. Let the assumptions of Proposition 9 be satisfied, and consider alternative specifications of monetary policy $\psi$ for a given vector $\boldsymbol{\delta}$. In addition, suppose that $v(y ; \bar{\xi})=$ $\nu \cdot y$ for some $\nu>0$ (that may depend on $\bar{\xi}$ ), and that the disturbance $\phi(0)$ is such that the sectors that are borrowing-constrained in period zero when $\psi=1$ (for the given specification of $\boldsymbol{\delta})$ imply a partition of the $\boldsymbol{A}$ matrix in which $\boldsymbol{A}_{C U}=\mathbf{0}$. Then $W_{0}$ is maximized when $\psi=1$; that is, if there is no response of monetary policy to the disturbance.

Note that the hypothesis about $\boldsymbol{A}_{C U}$ is satisfied by the examples shown in Figure 3, for any value of $a(0)>0$. Thus these are examples in which a reduction of $i(0)$ would reduce welfare, even when $a(0)<\hat{a}_{4}$, so that fiscal transfers are insufficient to achieve the optimal allocation of resources.

\section{Conclusion}

Our results imply that common views about the relative importance of interest-rate policy and fiscal transfers as tools of macroeconomic stabilization require revision in the case of an economic crisis resulting from a severely asymmetric disturbance, such as the COVID-19 pandemic. It is often thought that the existence of "Keynesian unemployment" provides a prima facie case for the desirability of interest-rate cuts to increase aggregate demand. Instead, we have seen that it is possible for a disturbance to result in inefficiently low activity in at least some parts of the economy (and inefficient over-consumption nowhere), in the absence of a policy response, and yet for the situation to be one in which the level of real interest rates required to support an efficient allocation of resources (assuming a suitable distribution of income) has not fallen as a result of the disturbance. 
The contraction of economic activity relative to the efficient pattern may result not from real interest rates being too high, but rather from an effective demand failure, owing to a disruption of the circular flow of payments between different parts of the economy. In such a situation, the policy response that can achieve the ex-ante optimal outcome is one that arranges for appropriately targeted lump-sum transfers in response to the disturbance, without any reduction of interest rates necessarily being needed at all (Proposition 5).

Of course, our model does not incorporate all of the channels through which monetary policy might be beneficial in response to a pandemic shock. ${ }^{43}$ But some of the ways in which lower interest rates could be beneficial involve effects that could also be achieved through other means, and that might better be achieved without also changing intertemporal relative prices. For example, in a more elaborate model, cutting the real interest rate should raise a variety of asset prices, and increased asset values might relax the financing constraints of the households or firms that own these assets. Borrowing constraints are at the heart of the problem of effective demand failure emphasized in this paper, and so one might argue that increased asset valuations should be helpful. Yet there are also other ways in which policy can increase the availability of credit to borrowing-constrained parts of the economy; and an advantage of direct credit policy (in addition to the fact that one does not distort intertemporal relative prices for parts of the economy that are not borrowing-constrained) is that it can be better targeted to the parts of the economy that are most impacted by the disruption of the usual circular flow of payments.

We should also stress that our analysis here concerns the role of interest-rate policy as a tool for regulating aggregate demand. Nothing in this paper should be taken to challenge the role of central banks as a lender of last resort, in the case of threats to financial stability. Threats to financial stability are likely to arise in the case of severe recessions, regardless of the nature of the disturbance that causes economic activity to contract. The model here abstracts from such issues, but is not intended to minimize them. We are concerned here only with the use of monetary policy to address an aggregate demand shortfall, in circumstances where financial institutions remain largely sound. ${ }^{44}$

Nor does our model imply that interest-rate reductions are never appropriate in response to a pandemic shock. Under certain relatively special circumstances (e.g., the cases described in Propositions 5 and Corollaries 5 and 7 ), we have seen that it is not ex-ante desirable for interest rates to be reduced at all; but more generally, a modest reduction in interest rates can increase ex-ante welfare, in the absence of a sufficiently aggressive (or sufficiently welltargeted) fiscal policy response. The more important point is that whereas a real interest-rate reduction of the right size should be able to completely solve the problems created by purely aggregate disturbances (Proposition 1), what one can hope to achieve with interest-rate policy alone is more limited in the case of a shock with significantly asymmetric effects.

\footnotetext{
${ }^{43}$ For example, Guerrieri et al. (2020) discuss a number of channels through which an interest-rate reduction might be helpful as a response to a pandemic, that are not present in the deliberately simplified model presented here.

${ }^{44}$ For example, we do not mean to question the appropriateness of the Federal Reserve's market interventions in March-April 2020, to address the disappearance of liquidity in the U.S. Treasury market at the onset of the COVID-19 pandemic (Fleming, 2020). But Treasury market liquidity had returned to 2019 levels within a few weeks; the Fed's aggressive monetary accommodation since then has instead been motivated by a concern to maintain aggregate demand.
} 
In particular, it will be a mistake to assume that if a modest interest-rate reduction does not eliminate all under-employment of productive resources, this means that even deeper real interest-rate reductions (perhaps through unconventional policies) are called for. Our examples show that the ex-ante optimal degree of interest-rate reduction can be reached long before inefficient under-production and under-consumption have been eliminated in all sectors. Instead, there is an important role for fiscal transfers as a response to significantly asymmetric disturbances, for which interest-rate policy does not provide a close substitute.

And we should be clear that in our analysis there remains an important role for interestrate policy as a tool of economic stabilization. Of course, all economic disturbances are at least somewhat asymmetric in their effects on different parts of the economy; "purely aggregate" disturbances of the kind assumed in Proposition 1 do not actually occur. ${ }^{45}$ However, actual disturbances can be decomposed into an aggregate component (that by itself would create no imbalance in the circular flow of payments) and an asymmetric component; and interest-rate policy should be the tool of choice to respond to the aggregate component, as in the situation described in Proposition 1. If this is the more important part of how a given disturbance affects the economy, interest-rate policy may correspondingly be the main policy response that is needed.

Indeed, in the case of sufficiently small asymmetric disturbances, there may be no need for any fiscal transfers in response to the shock in order for the ex-ante optimal allocation of resources to be achieved, at least in the $\beta \rightarrow 1$ limit, as noted in our discussion of Proposition 7. We could add aggregate disturbances to the situation assumed in the discussion following Proposition 7, and conclude that the first-best outcome can be achieved with an appropriate monetary policy response, but with no response of either fiscal policy or credit policy. Thus there is a range of possible circumstances in which the conventional view of stabilization policy, according to which interest-rate policy alone should be used, will not be off-base. But there are other situations, vividly illustrated by the COVID-19 crisis, in which this view is quite inadequate. A more complete theory of stabilization policy, that also allows for the possibility of effective demand failures, is badly needed.

One consequence should be a greater willingness to use fiscal transfers as a tool of stabilization policy, at least under some circumstances. But this is not the only way in which policy can be adjusted in order to minimize the distortions created by periodic failures of effective demand. Our model implies that the size of the asymmetric disturbances required to create a situation in which fiscal transfers and/or credit policy will be needed in order to prevent borrowing constraints from distorting spending patterns depends on the quantity of liquid assets held by the various economic units, that can serve to buffer transitory variations in the flow of payments. And while the equilibrium distribution of those liquid assets depends on the choices made by the various individual economic units in the economy, in our model the aggregate supply of them depends on the size of the public debt.

Thus maintaining a larger real public debt has the virtue of reducing the extent to which financing constraints distort the equilibrium allocation of resources, as argued in Woodford (1990). Of course, a higher real public debt also has costs, when the revenues required to

\footnotetext{
${ }^{45}$ It should also be noted that when we refer to "asymmetric disturbances," the differential effects need not only be differences across sectors or industries; they might be asymmetric effects on different regions, or on different occupational categories within an industry.
} 
service it can only be raised using distorting taxes; thus a balance must be struck between these costs and the benefits resulting from a higher average level of liquid balances. But it may be an important mistake to consider the optimal level of public debt without taking into account the advantages for macroeconomic stability of making it possible for people to maintain a higher level of liquid asset balances. 


\section{References}

[1] Acemoglu, Daron, Vasco M. Carvalho, Asuman Ozdaglar, and Alireza Tahbaz-Salehi, "The Network Origins of Aggregate Fluctuations," Econometrica 80: 1977-2016 (2012).

[2] Acemoglu, Daron, Asuman Ozdaglar, and Alireza Tahbaz-Salehi, "Systemic Risk and Stability in Financial Networks," American Economic Review 105: 564-608 (2015).

[3] Araújo, Aloísio P., and Vitor C. Costa, "Bankruptcy Law as an Alternative to Fiscal Policy in a Woodford Model with Pandemic Shock," working paper, IMPA, Rio de Janeiro, April 2021.

[4] Auclert, Adrien, Matthew Rognlie, and Ludwig Straub, "The Intertemporal Keynesian Cross," NBER Working Paper no. 25020, September 2018.

[5] Auerbach, Alan J., Yuriy Gorodnichenko, and Daniel Murphy, "Fiscal Policy and COVID19 Restrictions in a Demand-Determined Economy," NBER Working Paper no. 27366, June 2020.

[6] Balasko, Yves, The Equilibrium Manifold: Postmodern Developments in the Theory of General Economic Equilibrium, MIT Press, 2009.

[7] Baqaee, David Rezza and Emmanuel Farhi, "Supply and Demand in Disaggregated Keynesian Economies with an Application to the Covid-19 Crisis," NBER Working Paper no. 27152, revised June 2020.

[8] Bigio, Saki, and Jennifer La'O, "Distortions in Production Networks," Quarterly Journal of Economics 135: 2187-2253.

[9] Bigio, Saki, Mengbo Zhang, and Eduardo Zilberman, "Transfers vs. Credit Policy: Macroeconomic Policy Trade-offs During Covid-19," NBER Working Paper no. 27118, May 2020.

[10] Caballero, Ricardo J., and Alp Simsek, "A Model of Asset Price Spirals and Aggregate Demand Amplification of a 'Covid-19' Shock," NBER working paper no. 27044, revised May 2020.

[11] Cavalcante, Renato L.G., Yuxiang Shen, and Sławomir Stańczak, "Elementary Properties of Positive Concave Mappings with Applications to Network Planning and Optimization," IEEE Transactions on Signal Processing 64: 1774-1783 (2016).

[12] Céspedes, Luis Felipe, Roberto Chang, and Andrés Velasco, "The Macroeconomics of a Pandemic: A Minimalist Model," NBER Working Paper no. 27228, May 2020.

[13] Coibion, Olivier, Yuriy Gorodnichenko, and Michael Weber, "How Did U.S. Consumers Spend Their Stimulus Payments?" NBER Working Paper no. 27693, August 2020.

[14] Danieli, Ana, and Jane Olmstead-Rumsey, "Sector-Specific Shocks and the Expenditure Elasticity Channel During the COVID-19 Crisis," working paper, Northwestern University, May 2020. 
[15] Elliott, Matthew, Benjamin Golub, and Matthew O. Jackson, "Financial Networks and Contagion," American Economic Review 104: 3115-3153 (2014)

[16] Elliott, Matthew, Benjamin Golub, and Matthew V. Leduc, "Supply Network Formation and Fragility," arXiv: 2001.03853v4, posted February 19, 2021.

[17] Fleming, Michael, "Treasury Market Liquidity and the Federal Reserve During the COVID-19 Pandemic," Federal Reserve Bank of New York Liberty Street Economics, May 29, 2020.

[18] Gantmacher, F.R., The Theory of Matrices, volume II, Providence: AMS Chelsea Publishing, 1959.

[19] Ghassibe, Mishel, "Monetary Policy and Production Networks: An Empirical Investigation," Journal of Monetary Economics 119: 21-39 (2021).

[20] Goodman, Laurie, and Dan Magder, "Avoiding a COVID-19 Disaster for Renters and the Housing Market," Urban Institute policy brief, April 2020.

[21] Gopal, Prashant, "NYC Rental Market Pushed to Breaking Point by Tenant Debts," Bloomberg Businessweek, posted July 8, 2020.

[22] Guerrieri, Veronica, Guido Lorenzoni, Ludwig Straub, and Iván Werning, "Macroeconomic Implications of COVID-19: Can Negative Supply Shocks Cause Demand Shortages?" NBER Working Paper no. 26918, April 2020.

[23] Keynes, John Maynard, The General Theory of Employment, Interest and Money, London: MacMillan, 1936.

[24] La'O, Jennifer, and Alireza Tahbaz-Salehi, "Optimal Monetary Policy in Production Networks," working paper, Columbia University, July 2021.

[25] Leijonhufvud, Axel, "Effective Demand Failures," Swedish Journal of Economics 75: 27-48 (1973).

[26] Milne, Alistair, "A Critical Covid 19 Economic Policy Tool: Retrospective Insurance," working paper, Loughborough University, UK, posted on SSRN, March 2020.

[27] Oh, Hyunseung, and Ricardo Reis, "Targeted Transfers and the Fiscal Response to the Great Recession," Journal of Monetary Economics 59: S50-S64 (2012).

[28] Ozdagli, Ali, and Michael Weber, "Monetary Policy through Production Networks: Evidence from the Stock Market," NBER Working Paper no. 23424, May 2017.

[29] Pastén, Ernesto, Raphael Schoenle, and Michael Weber, "The Propagation of Monetary Policy Shocks in a Heterogeneous Production Economy," Journal of Monetary Economics 116: 1-22 (2020).

[30] Rubbo, Elisa, "Networks, Phillips Curves, and Monetary Policy," working paper, Harvard University, June 2020. 
[31] Saez, Emmanuel, and Gabriel Zucman, "Keeping Business Alive: The Government Will Pay," posted online at http://gabriel-zucman.eu/files/coronavirus2.pdf, March 2020.

[32] Woodford, Michael, "Public Debt as Private Liquidity," American Economic Review 80(2): 382-388 (1990).

[33] Woodford, Michael, Interest and Prices: Foundations of a Theory of Monetary Policy, Princeton: Princeton University Press, 2003.

[34] Woodford, Michael, "Effective Demand Failures and the Limits of Monetary Stabilization Policy," NBER Working Paper no. 27768, September 2020. 


\title{
For Online Publication \\ APPENDIX
}

\section{"Effective Demand Failures and the Limits of Monetary Stabilization Policy"}

\author{
Michael Woodford, Columbia University
}

Here we present additional details of the arguments in the main text, and proofs of the stated lemmas and propositions.

\section{A Welfare Analysis: Preliminary Results}

This section provides proofs of the main results in section 1 .

\section{A.1 Proof of Lemma 1}

As explained in the main text, in the case of any rotationally-invariant allocation of resources, if we let $U^{j}(t ; \xi, \phi)$ be the flow utility (1.2) in the case of disturbances $(\xi, \phi)$, we must have

$$
U^{j}(t ; \xi, R \phi)=U^{j-1}(t ; \xi, \phi)
$$

for each sector $j$. Then consider the contribution to the ex-ante expected value of (1.1) from the terms corresponding to the different possible outcomes in a particular rotation family (1.6). Because the $N$ different outcomes must each have the same ex-ante probability, the contribution from these terms must be proportional to their equally-weighted average,

$$
\bar{U}^{j} \equiv \frac{1}{N} \sum_{h=0}^{N-1} \sum_{t=0}^{\infty} \beta^{t} U^{j}\left(t ; \xi, R^{h} \phi\right)
$$

But it follows from (A.1) that

$$
U^{j}\left(t ; \xi, R^{h} \phi\right)=U^{j-1}\left(t ; \xi, R^{h-1} \phi\right)=U^{j-2}\left(t ; \xi, R^{h-2} \phi\right)=U^{j-h}(t ; \xi, \phi)
$$

for any integer $h$. Hence we can alternatively write

$$
\bar{U}^{j}=\frac{1}{N} \sum_{h=0}^{N-1} \sum_{t=0}^{\infty} \beta^{t} U^{j-h}(t ; \xi, \phi)=\frac{1}{N} \sum_{t=0}^{\infty} \beta^{t} \sum_{i=1}^{N} U^{i}(t ; \xi, \phi) .
$$

The final expression on the right in (A.2) is independent of the sector $j$ for which we compute ex-ante expected utility. Hence units in all sectors agree about the ex-ante ranking of alternative feasible rotationally-invariant allocations of resources, as stated in the lemma. The welfare criterion stated in the lemma is just $N$ times the final expression on the right in (A.2). 


\section{A.2 Proof of Lemma 2}

For given disturbance sequences $(\xi, \phi)$, we wish to find the consumption allocation $\left\{c_{k}^{j}(t)\right\}$ and production plan $\left\{y_{k}(t)\right\}$ for all $t \geq 0$ that maximizes (1.7), subject to the constraints that

$$
\sum_{j} c_{k}^{j}(t)=y_{k}(t)
$$

for each sector $k$ at each date $t$. Note that the welfare measure (1.7) consists of a sum of separate terms for each good $k$ at each date $t$. Since the constraints (A.3) each also involve only the production and consumption of a single good $k$ at a single date $t$, we can separate the optimization problem into a set of independent problems, one for each good $k$ and each date $t$.

If $k$ is a good for which $\phi_{k}(t)=0$, then the static optimization is simply the choice of $y_{k}(t)$ to minimize the disutility of supply $v\left(y_{k}(t) ; \xi_{t}\right)$. The solution is obviously $y_{k}(t)=0$, which then implies that the only feasible consumption allocation involves $c_{k}^{j}(t)=0$ for all $j$ as well. (Thus in the example of a "pandemic shock" discussed in the main text, where $\phi_{1}(0)=0$, it is optimal for there to be no production or consumption of good 1 in period 0 .)

If instead $\phi_{k}(t)>0$, the static optimization problem requires that we choose $y_{k}(t)$ and the $\left\{c_{k}^{j}(t)\right\}$ for $j=1, \ldots, N$ to maximize

$$
\sum_{h \in H} \phi_{k}(t) \alpha_{h} u\left(c_{k}^{k-h}(t) /\left(\alpha_{h} \phi_{k}(t)\right) ; \xi_{t}\right)-v\left(y_{k}(t) ; \xi_{t}\right)
$$

subject to the constraint (A.3). Substituting the left-hand side of (A.3) for $y_{k}(t)$ in (A.4), we obtain an objective that is purely a function of the consumption allocation. We further note that this function is monotonically decreasing in $c_{k}^{k-h}(t)$, for any $h \notin H$ (if such sectors exist). Hence the optimum must involve $c_{k}^{k-h}(t)=0$ for any $h \notin H$. With this substitution, we are left with an objective that is a function of the quantities $\left\{c_{k}^{k-h}(t)\right\}$ for $h \in H$,

$$
\sum_{h \in H} \phi_{k}(t) \alpha_{h} u\left(c_{k}^{k-h}(t) /\left(\alpha_{h} \phi_{k}(t)\right) ; \xi_{t}\right)-v\left(\sum_{h \in H} c_{k}^{k-h}(t) ; \xi_{t}\right)
$$

Because this last objective is a strictly concave function of its arguments, it has a unique optimum characterized by the first-order conditions. Moreover, the Inada conditions on the functions $u, v$ imply that there cannot be a corner solution; hence we need consider only the first-order conditions for an interior maximum. These require that $\left.u^{\prime}\left(c^{k-h}(t) / \alpha_{h} \phi_{k}(t)\right) ; \xi_{t}\right)$ be the same quantity for each $h \in H$. Since $u^{\prime}\left(c ; \xi_{t}\right)$ is a monotonically decreasing function of $c$, this in turn implies that $c^{k-h}(t) / \alpha_{h} \phi_{k}(t)$ must be the same for each $h \in H$. This in turn is only consistent with (A.3) if $c_{k}^{k-h}(t)=\alpha_{k-h} y_{k}(t)$ for each $h \in H$. Since the same expression holds for $h \notin H$ as well (where it simply states that $c_{k}^{k-h}(t)=0$ ), the optimal consumption allocation must satisfy (1.9) for all $j$, as stated in the lemma.

Finally, using this result, we can rewrite the objective (A.4) as a function of $y_{k}(t)$ alone, obtaining

$$
\phi_{k}(t) u\left(y_{k}(t) / \phi_{k}(t) ; \xi_{t}\right)-v\left(y_{k}(t) ; \xi_{t}\right) .
$$

The optimal output level is the $y_{k}(t)$ that maximizes (A.5). Since the objective involves only $\phi_{k}(t)$ and the disturbances $\xi_{t}$, the solution is of the form $y_{k}(t)=y^{*}\left(\phi_{k}(t)\right.$; $\left.\xi_{t}\right)$, where 
the function $y^{*}(\phi ; \xi)$ is the same for any sector $k$. Moreover (A.5) is again a strictly concave function, and the Inada conditions imply that we must have an interior optimum. There is thus a unique solution $y^{*}(\phi ; \xi)$, implicitly defined by the first-order condition (1.8) stated in the lemma.

This establishes Lemma 2. In addition, differentiation of (1.8) implies that

$$
\frac{\partial y^{*}}{\partial \phi}=\frac{y^{*}}{\phi} \frac{-u^{\prime \prime} / \phi}{v^{\prime \prime}-u^{\prime \prime} / \phi}>0
$$

so that $y^{*}$ is a monotonically increasing function of $\phi$, as stated in the main text. In the limit as $\phi \rightarrow 0$, the first term in (A.5) approaches zero regardless of the value of $y_{k}(t)$; hence the objective approaches a monotonically decreasing function of $y_{k}(t)$ for all $y_{k}(t)>0$, which is maximized when $y_{k}(t)=0$. Hence $y^{*} \rightarrow 0$ as $\phi \rightarrow 0$, for any disturbances $\xi$.

\section{A.3 Proof of Proposition 1}

The first-best optimal allocation of resources in this case has already been established in Lemma 2:

$$
y_{k}(t)=y_{t}^{*}, \quad c_{k}^{j}(t)=\alpha_{k-j} \cdot y_{k}(t)
$$

for all $j, k$, and all $t \geq 0$. We wish to prove that this resource allocation, together with prices

$$
p_{k}(t)=P^{*}(t), \quad 1+i(t)=\left(1+r_{t}^{*}\right) \frac{P^{*}(t+1)}{P^{*}(t)}
$$

for all $k$ and all $t \geq 0$, represents an equilibrium.

We first observe the prices and quantities specified in (A.6)-(A.7) imply that sector $j$ 's end-of-period asset position each period will satisfy $b^{j}(t)=a^{j}(t)$, as a consequence of (1.11). Substitution of this conclusion into (1.13) implies that

$$
a^{j}(t+1)=(1+i(t)) a^{j}(t)-\tau(t+1)
$$

for each $t \geq 0$. Then under the hypothesis that $a^{j}(0)=a(0) / N$ and that $\tau(t+1)$ each period is consistent with the fiscal authority's target path for the public debt, the assumed behavior implies that $a^{j}(t)=a(t) / N$ for all $t \geq 0$. This in turn implies that $b^{j}(t)=a(t) / N>0$ each period, so that the hypothesized spending plan is consistent with the borrowing constraint (1.12) each period. Hence the hypothesized spending plan represents a feasible plan for units in sector $j$. We next show that it is their optimal plan, i.e., that it is the feasible plan with the highest value for the objective (1.1).

Conditions (1.11) and (1.13) imply a law of motion for the nominal asset position of the form

$$
\frac{a^{j}(t+1)}{P(t+1)}=(1+i(t)) \frac{P(t)}{P(t+1)}\left[\frac{a^{j}(t)}{P(t)}+\frac{p_{j}(t) y_{j}(t)-\sum_{k} p_{k}(t) c_{k}^{j}(t)}{P(t)}\right]-\frac{\tau(t+1)}{P(t+1)}
$$

for each $t \geq 0$. The prices and interest rates assumed in (A.7) imply that in the conjectured equilibrium, this can be written in the form

$$
\beta \rho_{t+1}\left[\frac{a^{j}(t+1)}{P^{*}(t+1)}+\frac{\tau(t+1)}{P^{*}(t+1)}\right]=\rho_{t}\left[\frac{a^{j}(t)}{P^{*}(t)}+y_{j}(t)-\sum_{k} c_{k}^{j}(t)\right],
$$


where

$$
\rho_{t} \equiv u^{\prime}\left(y_{t}^{*} ; \xi_{t}\right)
$$

using the definition of the natural rate of interest in (1.17). Multiplying both sides of this equation by $\beta^{t}$ and summing for values of $t$ from 0 to $T-1$ yields the condition

$$
\beta^{T} \rho_{T} \frac{a^{j}(T)}{P^{*}(T)}+\sum_{t=1}^{T} \beta^{t} \rho_{t} \frac{\tau(t)}{P^{*}(t)}=\rho_{0} \frac{a^{j}(0)}{\bar{p}}+\sum_{t=0}^{T-1} \beta^{t} \rho_{t}\left[y_{j}(t)-\sum_{k} c_{k}^{j}(t)\right],
$$

which any feasible spending plan must satisfy for any $T \geq 1$.

We further note that because $\underline{b}^{j}(T-1)=0,(1.12)$ and (1.13) imply that $a^{j}(T) \geq$ $-\tau(T) / N$ under any feasible plan. In addition, (1.15) together with (1.19) implies that under any policy of the kind hypothesized, we must have

$$
\lim _{T \rightarrow \infty} \beta^{T} \rho_{T} \frac{\tau(T)}{P^{*}(T)}=0
$$

so that any feasible plan must satisfy

$$
\lim _{T \rightarrow \infty} \beta^{T} \rho_{T} \frac{a^{j}(T)}{P^{*}(T)} \geq 0
$$

Together with the fact that (A.9) must hold for arbitrary $T$, this implies that any feasible spending plan must satisfy the integrated intertemporal budget constraint

$$
\sum_{t=0}^{\infty} \beta^{t} \rho_{t} \sum_{k} c_{k}^{j}(t) \leq \rho_{0} \frac{a^{j}(0)}{\bar{p}}+\sum_{t=0}^{\infty} \beta^{t} \rho_{t} y_{j}(t)-\sum_{t=1}^{\infty} \beta^{t} \rho_{t} \frac{\tau(t)}{P^{*}(t)} .
$$

Moreover, under the hypothesized spending plan, $a^{j}(t)=a(t) / N$ each period; (1.19) then implies that under this plan, condition (A.11) holds with equality, and hence (A.12) is satisfied with equality.

We next show that the hypothesized spending plan is the optimal one for a unit in sector $j$, among all plans consistent with the constraint (A.12). This is a problem of maximizing a concave objective (1.1) subject to a single linear inequality constraint. Moreover, the fact that goods $k \notin K_{j}(t)$ have positive prices, but have no effect on the objective (1.1) makes it obvious that the optimal solution requires that $c_{k}^{j}(t)=0$ for any $k \notin K_{j}(t)$, as is true of the hypothesized spending plan (A.6). It then remains only to show that the hypothesized plan is optimal among all those satisfying the constraint that $c_{k}^{j}(t)=0$ for all $k \notin K_{j}(t)$.

We then seek to maximize a function that is increasing in each of its arguments $c_{k}^{j}(t)$ (for $k \in K_{j}(t)$ ), and strictly concave. The fact that the objective is increasing requires that the optimal plan must satisfy the constraint (A.12) with equality, but this is true of the hypothesized plan for each sector $j$, as just shown. In addition, the strict concavity of the objective implies that there must be a unique optimum, characterized by the firstorder conditions. The Inada conditions on the utility function $u(c ; \xi)$ further imply that the optimal plan must involve positive consumption of each of the goods $k \in K_{j}(t)$ in each period $t \geq 0$. It follows that a consumption plan $\left\{c_{k}^{j}(t)\right\}$ is optimal if and only if, in addition 
to satisfying the budget constraint (A.12) with equality, the marginal rate of substitution between any two goods is equal to their relative price.

The conjectured prices and quantities (A.6)-(A.7) imply that for any two goods $k, k^{\prime} \in$ $K_{j}(t)$ in some period $t$, we have

$$
\frac{u^{\prime}\left(c_{k}^{j}(t) / \alpha_{k-j} ; \xi_{t}\right)}{\left.u^{\prime}\left(c_{k^{\prime}}^{j}(t) / \alpha_{k^{\prime}-j}\right) ; \xi_{t}\right)}=\frac{\rho_{t}}{\rho_{t}}=1=\frac{P^{*}(t)}{P^{*}(t)}=\frac{p_{k}(t)}{p_{k^{\prime}}(t)}
$$

so that the intra-temporal optimality condition is satisfied. The marginal utility of an additional unit of expenditure in period $t$ is then equal to $u^{\prime}\left(y_{t}^{*} ; \xi_{t}\right) / P^{*}(t)$, regardless of which good $k \in K_{j}(t)$ it is spent on. Furthermore, the conjectured prices and quantities (A.6)-(A.7) imply that for any two successive periods $t, t+1$,

$$
\beta \frac{\rho_{t+1}}{\rho_{t}}=\frac{1}{1+r_{t}^{*}}=\frac{1}{1+i(t)} \frac{P(t+1)}{P(t)}
$$

so that the inter-temporal optimality condition is satisfied as well.

Thus all necessary and sufficient conditions are satisfied to establish that given the conjectured prices, the hypothesized spending plan is optimal among all plans consistent with the budget (A.12). Of course, this last constraint is a weaker condition than the full set of requirements for a spending plan to be feasible (in particular, it neglects the borrowing constraints), so the set of feasible plans is a proper subset of the set of plans consistent with (A.12). However, we have already verified that the hypothesized spending plan satisfies all of the conditions for feasibility (in particular, it also satisfies the borrowing constraint (1.12) each period); hence optimality among all plans in the larger set is a sufficient condition to establish optimality among those plans in the smaller set of feasible plans. We have therefore established that the hypothesized spending plan for each sector $j$ is optimal, given the conjectured prices.

The conjectured paths $\left\{y_{k}(t)\right\}$ for the production levels also satisfy (1.14) for each sector $k$ at each date $t \geq 0$. We must further show that the conjectured goods prices for dates $t \geq 1$ are market-clearing prices. This follows from (1.14), if for each sector $k, y_{k}(t)$ is the quantity that units in sector $k$ wish to supply, taking the price at which they can sell as given. Since we have already shown that the marginal utility of additional nominal income in period $t$ is $u^{\prime}\left(y_{t}^{*} ; \xi_{t}\right) / P^{*}(t)$ in any sector, the first-order condition for optimal supply in period $t$ is satisfied if and only if

$$
\frac{v^{\prime}\left(y_{k}(t) ; \xi_{t}\right)}{p_{k}(t)}=\frac{u^{\prime}\left(y_{t}^{*} ; \xi_{t}\right)}{P^{*}(t)} .
$$

But for any sector $k$, the conjectured values for $y_{k}(t)$ and $p_{k}(t)$ satisfy (A.13), because of (1.10).

We must also show that the conjectured path of interest rates $\{i(t)\}$ clears the market for liquid assets in each period $t \geq 0$. But we have already shown above that the hypothesized plan for units in any sector $j$ implies that $b^{j}(t)=a^{j}(t)=a(t) / N$ in each period, from which it follows that $\sum_{j} b^{j}(t)=a(t)$, and the asset market clears.

It thus remains only to verify that the conjectured paths are consistent with the government policies specified in the proposition. We have already discussed the consistency of these paths 
with the specified target path for the public debt and the specified borrowing limits. The conjectured prices (A.7) imply that in any period $t \geq 0$, the right-hand side of (1.16) is equal to

$$
\log \left(1+r^{*}\right)+\pi^{*}(t+1) \geq 0,
$$

where the sign is guaranteed by (1.18). Hence the specified monetary policy rule requires that $\log (1+i(t))$ equal the above expression, or alternatively, that

$$
1+i(t)=\left(1+r_{t}^{*}\right) \frac{P^{*}(t+1)}{P^{*}(t)}
$$

But this is exactly the path of interest rates specified in (A.7). Hence the prices and quantities specified in (A.6)-(A.7) constitute an equilibrium, under the policies specified in the proposition.

Note that the proposition asserts only that an equilibrium of this kind exists under these policies, and does not address the question whether this is the only possible equilibrium consistent with the policies. We could go further, and establish local determinacy of the proposed equilibrium, using the methods discussed in Woodford (2003) for the case of a single-sector model. We do not pursue such issues here, noting only that the issues connected to uniqueness of equilibrium in this model are similar to those that arise in the single-sector model.

\section{A.4 Proof of Proposition 2}

In this case, equilibrium prices are the same as in the one described in Proposition 1, and equilibrium interest rates are the same, except for $i(0)$. Equilibrium quantities are the same for all $t \geq 1$. In period $t=0$, quantities are instead given by

$$
y_{k}(0)=y(0), \quad c_{k}^{j}(0)=\alpha_{k-j} y(0)
$$

where $y(0)$ is the quantity implicitly defined by

$$
u^{\prime}\left(y(0) ; \xi_{0}\right)=\beta(1+i(0)) \frac{\bar{p}}{P^{*}(1)} u^{\prime}\left(y_{1}^{*} ; \xi_{1}\right)
$$

given the interest rate $i(0)$.

The proof that these prices and quantities represent a perfect foresight equilibrium under the assumed policy follows the same lines as in the proof of Proposition 1. First, we show that the proposed plan for each unit is feasible, given the assumed prices. The plans (A.14) imply that the circular flow of payments is again perfectly balanced in period zero, so that $b^{j}(0)=a^{j}(0)=a(0) / N$ for each $j$, just as in the case considered in Proposition 1 . This implies that the borrowing constraint (1.12) is satisfied in period zero. And because $b^{j}(0)$ is the same for all $j$, one must have $a^{j}(1)=a(1) / N$ for each $j$, which are the same initial asset positions at the beginning of period 1 as in Proposition 1. It then follows that the proposed plan satisfies the feasibility constraints in all periods $t \geq 1$, just as in the proof of Proposition 1. 
Next, we show that a feasible plan for each unit must satisfy an integrated intertemporal budget constraint of the form (A.12), where $\rho_{t}$ continues to be defined by (A.8) for all $t \geq 1$, but in period $t=0$ we instead define

$$
\rho_{0}=\beta(1+i(0)) \frac{\bar{p}}{P^{*}(1)} u^{\prime}\left(y_{1}^{*} ; \xi_{1}\right)
$$

which is only equal to the value defined in (A.8) if the interest rate is chosen so as to ensure that $y_{0}=y_{0}^{*}$. With this modification, the demonstration that a feasible plan satisfies the intertemporal budget constraint proceeds as in the proof of Proposition 1. Moreover, we can again show that the hypothesized plan for each unit satisfies this constraint with equality.

After this, in order to show that the proposed plan for each unit is the optimal plan among those consistent with the budget constraint (A.12), we need only to show that the marginal rates of substitution are all equal to the corresponding relative prices. In the case of marginal rates of substitution between goods in periods $t \geq 1$, all quantities and prices are the same as in Proposition 1, so that the equality of marginal rates of substitution and relative prices has already been shown. Condition (A.14) implies that the marginal rate of substitution between any two goods that units in sector $j$ consume in period zero is equal to their relative price (which is 1); and condition (A.15) implies that the marginal rate of substitution between real expenditure in period zero and real expenditure in period one is equal to the real interest rate between those two periods. Hence all of the necessary and sufficient conditions for optimality are satisfied. Then the fact that the plan is optimal among those consistent with the weaker constraint (A.12), while it also satisfies all of the additional conditions required for feasibility, together with the fact that all feasible plans must satisfy (A.12), implies that the proposed plan for each unit is optimal among all feasible plans.

Next, we have already shown in the proof of Proposition 1 that the conjectured paths $\left\{y_{k}(t)\right\}$ for the production levels also satisfy (1.14) for each sector $k$ at each date $t \geq 1$. Condition (A.14) implies that this is true in period $t=0$ as well. We have also already shown in the proof of Proposition 1 that the prices in all periods $t \geq 1$ are market-clearing prices. And once again, the hypothesized plan for units in any sector $j$ implies that $b^{j}(t)=$ $a^{j}(t)=a(t) / N$ in each period, from which it follows that $\sum_{j} b^{j}(t)=a(t)$, and the asset market clears in every period $t \geq 0$.

Finally, we have already shown in the proof of Proposition 1 that the conjectured prices and quantities in all periods $t \geq 1$ are consistent with the specification of monetary and fiscal policy in those periods. The prices and quantities assumed here for period $t=0$ are also consistent with our alternative specification of policy in period $t=0$. Hence all of the conditions for a perfect foresight equilibrium, under the assumed policy, have been shown to be satisfied.

In this equilibrium, the level of output $y_{0}$ is determined by (A.15). Since all quantities in this formula are taken as given, except the values of $y(0)$ and $i(0)$, the equation establishes a structural relationship between these two quantities. The left-hand side of (A.15) is a decreasing function of $y_{0}$, while the right-hand side is an increasing function of $i(0)$; it follows that $y(0)$ is a decreasing function of $i(0)$. When $i(0)$ takes the value specified by $(1.16)$, the equation implies that $y_{0}=y_{0}^{*}$. Hence $y_{0}$ is less than or greater than $y_{0}^{*}$ according to whether $i(0)$ is greater or less than the value specified by (1.16), as asserted in the proposition. 


\section{B Equilibrium with Asymmetric Disturbances}

Here we present proofs of the main results in section 2 of the main text.

\section{B.1 Proof of Lemma 3}

The proof that the prices and quantities described in the proposition constitute a flexibleprice perfect foresight equilibrium for all periods $t \geq 1$, taking as given the real financial wealth of each sector at the beginning of period 1, follows the same kind of reasoning as in the proof of Proposition 1.

First we verify that given the conjectured prices, the quantities described in the proposition represent a feasible plan for each sector $j$. The vector $\tilde{\boldsymbol{a}}(1)$, together with the target $a(1)$ for the public debt after taxes are collected at the beginning of period 1, implies that

$$
a^{j}(1)=\frac{a(1)}{N}+\left[\tilde{a}^{j}(1)-(1 / N) \sum_{\ell} \tilde{a}^{\ell}(1)\right] P^{*}(1)=\frac{a(1)}{N}+\frac{f^{j}}{1-\beta} P^{*}(1) .
$$

Then the fact that the stationary quantities $\left\{c_{k}^{j}\right\}$ and $y_{j}$ satisfy (2.23) implies that (1.11) is satisfied each period, if we further specify that

$$
a^{j}(t)=\frac{a(t)}{N}+\frac{f^{j}}{1-\beta} P^{*}(t), \quad b^{j}(t)=\frac{a(t)}{N}+\frac{\beta}{1-\beta} f^{j} P^{*}(t)
$$

for all $t \geq 1$. This path for $\left\{b^{j}(t)\right\}$ must also satisfy the borrowing constraint (1.12) each period, if the borrowing limit satisfies (2.22). Hence the hypothesized plan for sector $j$ is feasible, given the conjectured prices and the specified policy.

Next we show that any feasible plan for periods $t \geq 1$ must satisfy an integrated intertemporal budget constraint. We can proceed as in the proof of Proposition 1 to show that under any feasible plan for a unit in sector $j$, we must have

$$
\beta^{T-1} \frac{a^{j}(T)}{P^{*}(T)}+\sum_{t=1}^{T} \beta^{t-1} \frac{\tau(t)}{N P^{*}(t)}=\tilde{a}^{j}(1)+\sum_{t=1}^{T-1} \beta^{t-1}\left[q_{j}^{*} y_{j}(t)-\sum_{k} q_{k}^{*} c_{k}^{j}(t)\right],
$$

for any $T \geq 2$. (The factors $\left\{\rho_{t}\right\}$ are now omitted, because the absence of aggregate disturbances implies that now $\rho_{t}=\rho_{1}$ for all $t \geq 1$; but we must now take account of intra-period relative prices, assumed to equal 1 in (A.9).)

The argument after this step is slightly more complicated than in the proof of Proposition 1 , because we no longer assume that $\underline{b}^{j}(t)=0$ each period. However, (1.12) and (1.13) in the more general case imply that

$$
\frac{a^{j}(T)}{P^{*}(T)} \geq \frac{(1+i(T-1)) \underline{b}^{j}(T-1)}{P^{*}(T)}-\frac{\tau(T)}{N P^{*}(T)}=\beta^{-1} \frac{\underline{b}^{j}(T-1)}{P^{*}(T-1)} .
$$

Then the assumption (2.20) together with (A.10) guarantees that any feasible plan must satisfy (A.11), even under our more general assumption about the borrowing limits. ${ }^{46}$ This

\footnotetext{
${ }^{46}$ Because of this, the result in Proposition 1 could easily be generalized to allow the more flexible kind of borrowing limits assumed here.
} 
together with (B.2) then implies that any feasible plan must satisfy the integrated intertemporal budget constraint

$$
\sum_{t=1}^{\infty} \beta^{t-1} \sum_{k} q_{k}^{*} c_{k}^{j}(t) \leq \tilde{a}^{j}(1)+\sum_{t=1}^{\infty} \beta^{t-1}\left[q_{j}^{*} y_{j}(t)-\frac{\tau(t)}{N P^{*}(t)}\right] .
$$

In addition, (1.15) and (1.19) imply that

$$
\sum_{t=1}^{\infty} \beta^{t-1} \frac{\tau(t)}{P^{*}(t)}=\frac{(1+i(0)) a(0)}{P^{*}(1)}=\sum_{\ell=1}^{N} \tilde{a}^{\ell}(1) .
$$

Substituting this into the right-hand side of (B.3) and using the definition (2.24), the intertemporal budget constraint can alternatively be written

$$
\sum_{t=1}^{\infty} \beta^{t-1} \sum_{k} q_{k}^{*} c_{k}^{j}(t) \leq \sum_{t=1}^{\infty} \beta^{t-1} q_{j}^{*} y_{j}(t)+\frac{f^{j}}{1-\beta} .
$$

Thus under the assumptions of the proposition, any feasible plan for units in sector $j$ must satisfy (B.4). Moreover, it follows from (2.23) that the hypothesized stationary plan satisfies (B.4) with equality.

Next we show that the hypothesized plan for units in sector $j$ maximizes the terms in (1.1) for periods $t \geq 1$, among all plans consistent with (B.4). We have already shown that the hypothesized plan satisfies (B.4) with equality, so it remains only to show that all marginal rates of substitution are equal to the corresponding relative prices. The fact that the stationary allocation is a competitive equilibrium of the static model requires that

$$
\frac{u^{\prime}\left(c_{j+h}^{j} / \alpha_{h} ; \bar{\xi}\right)}{q_{j+h}^{*}}=\frac{v^{\prime}\left(y_{j} ; \bar{\xi}\right)}{q_{j}^{*}}
$$

for each $h \in H$ (i.e., for each of the goods that units in sector $j$ consume in periods $t \geq$ 1). This implies that all intra-temporal marginal rates of substitution are equal to the corresponding relative prices. We also note that the marginal utility of additional expenditure on the composite good in any period, for units in sector $j$, is given by (any of) the expressions in (B.5). Thus under the hypothesized plan, this marginal utility is constant over time, and the marginal rate of substitution between expenditure on the composite good in periods $t$ and $t+1$ (for any $t \geq 1$ ) is equal to $\beta^{-1}$. This is exactly the real interest rate between these periods, under the hypothesized prices, because of (2.25). Thus we verify all of the necessary and sufficient conditions for the hypothesized plan to maximize (1.1) among all plans consistent with (B.4). Since we have also shown that all feasible plans must satisfy (B.4), and that the hypothesized plan is feasible, it follows that the hypothesized plan maximizes (1.1) among all feasible plans.

Next we show that markets clear under the hypothesized plans for all sectors. The fact that goods markets clear each period follows from the fact that the intra-temporal resource allocation each period corresponds to a Walrasian competitive equilibrium of the static model. And condition (B.1) implies that

$$
\sum_{j=1}^{N} a^{j}(t)=a(t)+\frac{\sum_{j=1}^{N} f^{j}}{1-\beta} P^{*}(t)=a(t)
$$


each period, so that the asset market clears as well.

Finally, we show that the conjectured paths are consistent with the specification of policy. The only non-trivial condition to check is consistency with the Taylor rule (1.16) for interestrate policy. Because the inflation is consistent with the central bank's target in each of the periods $t \geq 1$ under the conjectured paths, the right-hand side of (1.16) corresponds simply to $P^{*}(t+1) /\left(\beta P^{*}(t)\right)$, which is greater than 1 under the assumption (1.18). Hence the assumed monetary policy rule requires that $1+i(t)$ equal this quantity each period, which is precisely what (2.25) assumes. Hence the conjectured paths are consistent with policy, and constitute a perfect foresight equilibrium under the assumed policy.

Moreover, in this equilibrium, the borrowing constraint (1.12) is not a binding constraint in any period $t \geq 1$, as we have shown that each unit's intertemporal plan would also be optimal if the unit were subject to only an integrated intertemporal budget constraint. Note also that our analysis implies that it would be possible to relax the borrowing limit $\underline{b}^{j}(t)$ in any period $t \geq 1$ without this implying any change in the equilibrium paths of prices or quantities.

\section{B.2 Proof of Lemma 4}

The function $V^{j}(\tilde{a} ; \tilde{\boldsymbol{a}}(1))$ is the maximum achievable value of the discounted utility in periods $t \geq 1$ for a unit in sector $j$ that carries real pre-tax wealth $\tilde{a}$ into period $t=1$, if the prices and interest rates are the ones associated with the stationary equilibrium determined by the aggregate vector of pre-tax wealths $\tilde{\boldsymbol{a}}(1)$. The envelope theorem then implies that the partial derivative of $V^{j}$ with respect to $\tilde{a}$, evaluated at $\tilde{a}=\tilde{a}^{j}(1)$, will equal the marginal utility of real expenditure in period 1 in the stationary equilibrium. Thus we must have

$$
\Lambda^{j}\left(\tilde{a}^{j}(1) ; \tilde{\boldsymbol{a}}(1)\right)=\frac{u^{\prime}\left(c_{k}^{j} / \alpha_{k-j} ; \bar{\xi}\right)}{q_{k}^{*}}=\frac{v^{\prime}\left(y_{j} ; \bar{\xi}\right)}{q_{j}^{*}},
$$

where we note that in the stationary equilibrium, the middle expression must have the same value for each $k$ such that $\alpha_{k-j}>0$, and the final expression must also have this same value, as a consequence of the first-order conditions for optimization by units in sector $j$.

Moreover, it follows from Lemma 3 that the allocation of resources in the stationary equilibrium depends only on the value of the vector $\boldsymbol{f}$ implied by the vector $\tilde{\boldsymbol{a}}(1)$. Thus both the second and third expressions in (B.6) are functions of $\boldsymbol{f}$ that are independent of the value of $\beta$, and we can define

$$
\Lambda^{* j}(\boldsymbol{f}) \equiv \frac{v^{\prime}\left(y_{j}(\boldsymbol{f}) ; \bar{\xi}\right)}{q_{j}^{*}(\boldsymbol{f})} .
$$

Here $y_{j}(\boldsymbol{f})$ means the output supply by sector $j$ in the static competitive equilibrium associated with the vector of net transfers $\boldsymbol{f}$, and $q_{j}^{*}(\boldsymbol{f})$ is the relative price of the sector $j$ good in that same equilibrium. Note that we have assumed that $\tilde{\boldsymbol{a}}(1)$ is such that $\boldsymbol{f} \in U$, so that we have a uniquely defined static competitive equilibrium with the vector $\boldsymbol{f}$. It then follows not only that $\Lambda^{* j}(\boldsymbol{f})$ is uniquely defined, but that it varies continuously with variation in the elements of $\boldsymbol{f}$. 
Our definition of the equilibrium selection has also assumed that when $\boldsymbol{f}=\mathbf{0}$, the static competitive equilibrium is the one in which $y_{j}=\bar{y}, q_{j}^{*}=1$ for each sector. Thus we have

$$
\Lambda^{* j}(\mathbf{0})=v^{\prime}(\bar{y} ; \bar{\xi})=u^{\prime}(\bar{y} ; \bar{\xi})
$$

And since $\Lambda^{* j}(\boldsymbol{f})$ must be a continuous function near $\boldsymbol{f}=\mathbf{0}$, we must have

$$
\lim _{\boldsymbol{f} \rightarrow \mathbf{0}} \Lambda^{* j}(\boldsymbol{f})=u^{\prime}(\bar{y} ; \bar{\xi})
$$

for each $j$, as asserted in the lemma.

\section{B.3 Proof of Proposition 3}

Here we consider equilibrium determination when $a(0)$ is arbitrarily close to zero (though we assume that $a(0)>0$ ), and $\underline{b}^{j}(0)=0$ for all $j$. In this case, satisfaction of the borrowing constraint (1.12) by all sectors requires that $0 \leq b^{j}(0) \leq a(0)$ for each sector, and hence that each element of $\boldsymbol{f}$ must satisfy the bounds

$$
-\frac{1}{N}(1-\beta) \frac{(1+i(0))}{P^{*}(1)} a(0) \leq f^{j} \leq \frac{N-1}{N}(1-\beta) \frac{(1+i(0))}{P^{*}(1)} a(0) .
$$

As $a(0) \rightarrow 0$, for fixed values of the other model parameters, both the upper and lower bounds converge to 0 . Hence we can assure that in equilibrium, all elements of $\boldsymbol{f}$ must be as close as may be desired to zero, by fixing a sufficiently small value for $a(0)$.

Lemma 4 then implies that the value of $\Lambda^{j}\left(\tilde{a}^{j}(1) ; \tilde{\boldsymbol{a}}(1)\right)$ must approach $u^{\prime}(\bar{y} ; \bar{\xi})$, so that under the assumption about $i(0)$ maintained in the proposition, the Euler condition (2.27) takes the simpler form $(2.29)$.

In addition, given that $\beta<1$, the bounds (B.7) can alternatively be written

$$
-\frac{1}{N} a(0) \leq b^{j}(0) \leq \frac{N-1}{N} a(0) .
$$

Thus we observe that in the limit as $a(0) \rightarrow 0$, the equilibrium value of each of the $\left\{b^{j}(0)\right\}$ must approach zero. We thus calculate the equilibrium in period zero for the limiting case in which we must have $b^{j}(0)=0$ for all $j$. It then follows, as discussed in the main text, that the vector $\boldsymbol{c}(0)$ of sectoral expenditure levels must satisfy $\boldsymbol{c}(0)=\boldsymbol{A c}(0)$.

We now show that under our assumptions, the matrix $\boldsymbol{A}$ must have a unique right eigenvector $\boldsymbol{\pi}$ with an associated eigenvalue of 1 . We first note that the definition of the matrix $\boldsymbol{A}$ in (1.5) implies that $A_{k j} \geq 0$ for all $k, j$, and that $\sum_{k=1}^{N} A_{k j}=1$ for every $j$, or in vector notation, that

$$
e^{\prime} \boldsymbol{A}=e^{\prime}
$$

This indicates that 1 must be an eigenvalue of the matrix $\boldsymbol{A}$, with $\boldsymbol{e}^{\prime}$ the associated left eigenvector. Any eigenvalue must also have at least one associated right eigenvector; thus it remains only to establish that the right eigenvector $\boldsymbol{\pi}$ is unique (up to normalization).

We observe from the properties noted in the previous paragraph that $\boldsymbol{A}$ is a non-negative matrix (Gantmacher, 1959, chap. XIII, Definition 1) that is furthermore a stochastic matrix 
(Definition 4). ${ }^{47}$ Any non-negative matrix necessarily has a maximal (Frobenius-Perron) eigenvalue $\bar{\lambda}$ with the properties that (i) $\bar{\lambda}$ is a non-negative real number, and (ii) $|\lambda| \leq \bar{\lambda}$ for all eigenvalues $\lambda$ of the matrix (where $|\lambda|$ denotes the modulus of an eigenvalue that may be complex); moreover, (iii) the left and right eigenvectors associated with the maximal eigenvalue are real-valued and non-negative in all elements (Gantmacher, Theorem 3). In the more specific case of a stochastic matrix, the maximal eigenvalue is 1 (Gantmacher, $p$. 83). The associated left eigenvector is $\boldsymbol{e}^{\prime}$, which is obviously non-negative in all elements; but there must be a (non-zero) right eigenvector $\boldsymbol{\pi}$ that is also non-negative in all of its elements. Because $\boldsymbol{\pi} \geq \mathbf{0}$, we can normalize the right eigenvector to satisfy $\boldsymbol{e}^{\prime} \boldsymbol{\pi}=1$.

To go further it is useful to write the matrix $\boldsymbol{A}$ in the normal form defined in Gantmacher (sec. XIII.4). ${ }^{48}$ This involves partitioning the $N$ sectors (the rows and columns of the matrix) into disjoint subsystems $\left\{S_{1}, \ldots, S_{s}\right\}$, each of which is irreducible, in the sense that any two sectors $j \neq z$ within the same subsystem can be linked by a sequence of sectors $(j, k, l, \ldots, y, z)$ all within the same subsystem, with the property that $j$ buys goods produced in $k, k$ buys goods produced in $l, \ldots$, and $y$ buys goods produced in $z$. We further define a subsystem as "isolated" if each of the sectors $j \in S_{i}$ spend only on products of sectors in subset $S_{i}$. Then Gantmacher shows that one can order the subsystems so that the first $g \geq 1$ of them are the (only) isolated subsystems; and the subsystems $S_{i}$ for $g+1 \leq i \leq s$ each have the property that each of the sectors $j \in S_{i}$ spends only on products produced in subsystems $S_{k}$ with $k \leq i$. Thus if one re-orders the sectors in accordance with this ordering of the subsystems, the matrix $\boldsymbol{A}$ has a normal form representation that is upper block-triangular, with all off-diagonal blocks being blocks of zeroes in the first $g$ block columns.

In the case that $\phi_{k}(0)>0$ for all $k$, our assumption that $\alpha_{1}>0$ implies that there is only one isolated subsystem, which is the complete system of all $N$ sectors (1 buys from 2 , which buys from $3, \ldots$, which buys from $N$, which buys from 1 ). Hence in this case, we must have $g=1, s=1$. The situation is only slightly more complicated if there exists a sector for which $\phi_{k}(0)=0$. We have assumed that there can be at most one such sector; let it be sector 1 (as in the numerical examples shown in Figure 2). In this case, we must have $g=1, s \geq 2$. The only isolated subsystem must be the one that contains sector $N$; the assumption that $\alpha_{1}>0$ implies that 1 buys from 2 which buys from ... which buys from $N-1$ which buys from $N$, so that any isolated subsystem must contain sector $N$. In addition, subsystem $S_{s}$ must consist solely of sector 1 , since no other sector buys anything from sector 1 (as a result of the pandemic); thus there must be at least two subsystems.

Gantmacher (Theorem 12) shows that a stochastic matrix $\boldsymbol{A}$ has a unique right eigenvector $\boldsymbol{\pi}$ with an associated eigenvalue of 1 if and only if $g=1$ in the normal form representation (i.e., there is a unique isolated subsystem). In this case the Frobenius-Perron eigenvector $\boldsymbol{\pi}$ corresponds to the uniquely defined stationary long-run probabilities of occupying the $N$ different states, if $\boldsymbol{A}$ is interpreted as the matrix of transition probabilities defining a homogeneous $N$-state Markov chain. The elements of this eigenvector satisfy $\pi_{j}>0$ for all $j \in S_{1}$, and $\pi_{j}=0$ for all other $j$. We have shown that this result necessarily applies to our model.

\footnotetext{
${ }^{47}$ More precisely, the transpose $\boldsymbol{A}^{\prime}$ is a stochastic matrix as defined in Gantmacher. Below we translate the properties of stochastic matrices established in Gantmacher into statements about the matrix $\boldsymbol{A}$.

${ }^{48}$ More precisely, we put $\boldsymbol{A}^{\prime}$ in the form shown in Gantmacher (p. 75).
} 
This unique solution for $\boldsymbol{\pi}$ allows us then to solve uniquely for the vector $\boldsymbol{c}(0)=\Omega \boldsymbol{\pi}$, where the value of $\Omega>0$ is given by (2.31), as explained in the main text, in order to satisfy (2.29). This establishes the proposition.

Because $\boldsymbol{c}(0)$ is a multiple of $\boldsymbol{\pi}$, it has the property that $c^{j}(0)>0$ for all $j$ in $S_{1}$, while $c^{j}(0)=0$ for all other sectors. For example, in the case of the uniform network structure shown in the left panel of Figure $1, S_{1}$ consists of sectors $\{2,3,4,5\}$, while $S_{2}$ consists of $\{1\}$. In the case of the chain structure shown in the right panel of Figure 1, instead (and regardless of the value of the parameter $\lambda$ ), the irreducible subsystems are $S_{1}=\{5\}$, $S_{2}=\{4\}, S_{3}=\{3\}, S_{4}=\{2\}$, and $S_{5}=\{1\}$, among which only $S_{1}$ is an isolated subsystem. This explains why, in Figure 2, we have $c^{j}(0)=0$ only for $j=1$ in the left panel, while instead $c^{j}(0)=0$ for all $j \leq 4$ in the right panel.

\section{B.4 Proof of Corollary 1}

We know from Lemma 2 that the first-best consumption level $c_{k}^{j}(0)$ of any good $k$ that is consumed by sector $j$ in period zero is given by $(1.9)$, where $y_{k}(0)=y^{*}\left(\phi_{k}(0) ; \bar{\xi}\right)$, and the function $y^{*}(\phi ; \xi)$ is implicitly defined by (1.8). We have also shown in section A.2 above that $y^{*}(\phi ; \xi)$ is an increasing function of $\phi$, for a given disturbance vector $\xi$. Because we have assumed that $v$ is at least weakly convex in $y, v^{\prime}\left(y^{*} ; \bar{\xi}\right)$ must be non-decreasing when $\phi$ increases. Thus the right-hand side of (1.8) must be non-decreasing, and so the left-hand side cannot decrease either. Because $u^{\prime}(c ; \bar{\xi})$ is a decreasing function of $c$, it follows that $y^{*} / \phi$ must be non-increasing when $\phi$ increases.

Then since the optimal level of production for sector $k$ satisfies $y^{*} / \phi=\bar{y}$ when $\phi_{k}(0)=1$, we must have $y^{*} / \phi \geq \bar{y}$ for all $0<\phi_{k}(0) \leq 1$. Thus the optimal level of production in sector $k$ is necessarily no smaller than $\phi_{k}(0) \bar{y}$. Combining (1.9) with this result, we conclude that the optimal consumption of good $k$ by sector $j$ must satisfy

$$
c_{k}^{j, o p t}(0) \geq \alpha_{k-j} \phi_{k}(0) \bar{y}
$$

But the quantity on the right-hand side here is the equilibrium consumption if the borrowing constraint does not bind for units in sector $j$, from (2.32). This in turn is an upper bound on equilibrium consumption, since a binding borrowing constraint can only reduce $c^{j}(0)$ and hence (because of (1.4) reduce sector $j$ consumption of all goods of which there is positive consumption. Thus we must have

$$
c_{k}^{j}(0)=A_{k j} c^{j}(0) \leq \alpha_{k-j} \phi_{k}(0) \bar{y} \leq c_{k}^{j, o p t}(0)
$$

for each good $k$. Thus no good can be consumed in an amount greater than the amount required for the first-best optimal allocation, as stated in the corollary.

Furthermore, if sector $j$ is borrowing-constrained in period zero, the first inequality in (B.9) must be a strict inequality. Thus in this case we must have $c_{k}^{j}(0)<c_{k}^{j, o p t}(0)$, as stated in the corollary (condition (i)). Finally, if $v$ is a strictly convex function of $y$, the function $v^{\prime}\left(y^{*} ; \bar{\xi}\right)$ must strictly increasing (and not just non-decreasing) when $\phi$ increases. From this we can conclude (by an argument parallel to the one made above, under the weaker assumption of weak convexity) that $y^{*} / \phi$ must be a decreasing function of $\phi$ (not just nonincreasing). This implies that $y^{*} / \phi \geq \bar{y}$ for all $0<\phi_{k}(0)<1$, from which we conclude that 
(B.8 must be a strict inequality for any $0<\phi_{k}(0)<1 .{ }^{49}$ This then implies that the last inequality in (B.9) must be a strict inequality, so that again we must have $c_{k}^{j}(0)<c_{k}^{j, o p t}(0)$, as stated in the corollary (condition (ii)).

\section{B.5 Algebra of the examples in Figure 2}

In the case of a uniform network structure (the left panel of Figure 2), $A_{k j}=1 /(N-1)$ for all $j$ and any $k \neq 1$; the Frobenius-Perron maximal right eigenvector is then easily seen to be

$$
\boldsymbol{\pi}=\left(\begin{array}{llll}
0 & 1 /(N-1) & \ldots & 1 /(N-1))^{\prime} .
\end{array}\right.
$$

(Because every sector spends the same amount in each sector $k \neq 1$, but nothing in sector 1, the eigenvector must have this property as well.) Furthermore, $\omega_{j}=(N-1) / N$ for all $j$. Hence the maximal value in the problem on the right-hand side of (2.31) is achieved by all sectors $j \neq 1$, and the equilibrium expenditure vector is given by

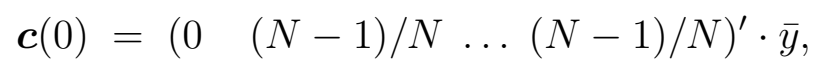

as shown in the left panel of Figure 2 for the case $N=5$. In this example, expenditure collapses completely in sector 1 (which no longer receives any income), but it is reduced in sectors $j \neq 1$ only to the extent that it is efficient for these sectors to reduce their spending (given that they no longer can or should buy sector-1 goods).

The collapse of effective demand is much more severe (and the inefficiency much greater) in the case of a "chain" network. In this case, one can show that the Frobenius-Perron maximal right eigenvector is given by

$$
\boldsymbol{\pi}=\left(\begin{array}{llll}
0 & \ldots & 0 & 1
\end{array}\right)^{\prime} .
$$

Sector 1 cannot spend at all, because it receives no income. Given that sector 1 cannot spend, sector 2 receives no income other than its own within-sector spending. But because sector 2 does not spend all of its income within-sector, an eigenvalue with eigenvector 1 must involve zero spending by this sector as well. Continuing iteratively in this way, one can show that every sector but sector $N$ must have zero expenditure.

The argument no longer goes through in the case of sector $N$, because - given that they can no longer buy sector 1 goods - units in sector $N$ spend all of their income within-sector. Hence all elements of $\boldsymbol{\pi}$ but the final one must equal zero. In the problem on the right-hand side of (2.31), sector $N$ achieves the maximum. Then given that $c^{* N}=\left(1-\alpha_{1}\right) \bar{y}=(1-\lambda) \bar{y}$, the equilibrium expenditure vector is given by

$$
\boldsymbol{c}(0)=\left(\begin{array}{llll}
0 & \ldots & 0 & 1-\lambda
\end{array}\right)^{\prime} \cdot \bar{y},
$$

as shown in the right panel of Figure 2 for the case $N=5, \lambda=0.8$.

\footnotetext{
${ }^{49}$ Note that (B.8) still holds with equality if $\phi_{k}(0)$ is equal to either 0 or 1 . In the former case, both sides are equal to zero; in the latter case, both sides are equal to $\alpha_{k-j} \bar{y}$. Note also that (B.8) also holds with equality for any good $k$ such that $\alpha_{k-j}=0$, so that sector $j$ does not wish to consume it, regardless of the value of $\phi_{k}(0)$.
} 
These two cases illustrate the two extremes with regard to the degree of collapse of aggregate expenditure and output in the limiting case in which $a(0) \rightarrow 0$. For a general network structure with a fraction $\alpha_{0}=1 / N$ of within-sector spending by all sectors (as in these examples), we can show that aggregate spending $c^{a g g}(0) \equiv \sum_{j=1}^{N} c^{j}(0)$ must fall within the bounds

$$
(1 / N) \bar{y} \leq c^{a g g}(0) \leq(N-2+(1 / N)) \bar{y}<(N-1) \bar{y}=y^{*} \equiv \sum_{j=1}^{N} c^{* j} .
$$

Here the lower bound is established by the fact that at least one sector must not be borrowingconstrained, and since that sector cannot be sector 1 , its first-best level of spending $c^{* j}$ must at least equal $\alpha_{0} \bar{y}=\bar{y} / N$. The upper bound is established by the fact that spending by sector 1 must be zero, and that spending in every other sector must be bounded above by $c^{* j}$, which cannot exceed $\bar{y}$ for any sector. Both of these bounds are achievable, since (as just shown) the chain network achieves the lower bound while the uniform network achieves the upper bound.

\section{B.6 Proof of Proposition 4}

We derive the solution to (2.35) and demonstrate its uniqueness in several steps. We begin by observing that there must be a unique solution, before deriving a closed-form expression for that solution.

\section{B.6.1 Existence of a unique solution to the "Keynesian cross"}

Let $\boldsymbol{\delta}>>\mathbf{0}$ be fixed, and consider the set of vectors $\boldsymbol{c}(0)$ that satisfy (2.35). We can show that there must be a unique solution using properties of positive concave mappings that are reviewed in Cavalcante et al. (2016). For any vector $\boldsymbol{c}(0)$, let $\boldsymbol{F}(\boldsymbol{c}(0))$ be the vector defined by the right-hand side of $(2.35)$; thus $\boldsymbol{F}(\cdot)$ maps $N$-vectors into $N$-vectors. If $\boldsymbol{\delta}(0)>>\mathbf{0}$, we can further show that $\boldsymbol{F}(\cdot)$ is a positive mapping, in the sense that for any $\boldsymbol{c}(0) \geq \mathbf{0}$, we have $\boldsymbol{F}(\boldsymbol{c}(0))>>\mathbf{0}$. Let $F_{j}(\cdot)$ be the $j$ th element of $\boldsymbol{F}(\cdot)$, that is, the implied value for $c^{j}(0)$. Then for each $j$, we need to show that for any $\boldsymbol{c}(0) \geq \mathbf{0}, F_{j}(\boldsymbol{c}(0))>0$. Since $\boldsymbol{A} \geq \mathbf{0}$, $\boldsymbol{c}(0) \geq \mathbf{0}$ implies that $\boldsymbol{A} \boldsymbol{c}(0) \geq \mathbf{0}$. Then under the hypothesis that $\boldsymbol{\delta}(0)>>\mathbf{0}$, we must have

$$
\frac{1}{\bar{p}} \boldsymbol{\delta}(0)+\boldsymbol{A} \boldsymbol{c}(0)>>\mathbf{0} .
$$

Thus the $j$ th element of this vector must be positive, for any $j$. Since $c^{* j}>0$ as well, the minimum of the two quantities must be positive. Thus $F_{j}(\boldsymbol{c}(0))$ is necessarily positive, as required.

We can further show that each of the functions $F_{j}(\cdot)$ is concave. This requires that for any vectors $\boldsymbol{c}_{1}, \boldsymbol{c}_{2}$, and any scalar $0 \leq \alpha \leq 1$,

$$
F_{j}\left(\alpha \boldsymbol{c}_{1}+(1-\alpha) \boldsymbol{c}_{2}\right) \geq \alpha F_{j}\left(\boldsymbol{c}_{1}\right)+(1-\alpha) F_{j}\left(\boldsymbol{c}_{2}\right)
$$


Given the definition of $F_{k}(\cdot)$ in $(2.35)$ as the minimum of two functions, this holds if and only if both of the inequalities

$$
\begin{gathered}
\frac{\delta^{j}}{\bar{p}}+\sum_{k} A_{j k}\left[\alpha c_{1}^{k}+(1-\alpha) c_{2}^{k}\right] \geq \alpha F_{j}\left(\boldsymbol{c}_{1}\right)+(1-\alpha) F_{j}\left(\boldsymbol{c}_{2}\right), \\
c^{* j} \geq \alpha F_{j}\left(\boldsymbol{c}_{1}\right)+(1-\alpha) F_{j}\left(\boldsymbol{c}_{2}\right)
\end{gathered}
$$

are necessarily satisfied. But inequality (B.11) follows from the fact that

$$
F_{j}\left(\boldsymbol{c}_{i}\right) \leq \frac{\delta^{j}}{\bar{p}}+\sum_{k} A_{j k} c_{i}^{k}
$$

for each of the cases $i=1,2$; and inequality (B.12) follows from the fact that

$$
F_{j}\left(\boldsymbol{c}_{i}\right) \leq c^{* j}
$$

for each of the cases $i=1,2$. Hence (B.10) is satisfied, and $F_{j}(\cdot)$ is a concave function for each $j$. This in turn means that $\boldsymbol{F}(\cdot)$ is a concave mapping.

Thus $\boldsymbol{F}(\cdot)$ is a positive concave mapping. Moreover, there exists a finite upper bound $\overline{\boldsymbol{c}}$ with the property that $\boldsymbol{F}(\boldsymbol{c}(0)) \leq \overline{\boldsymbol{c}}$ for all $\boldsymbol{c}(0) \leq \overline{\boldsymbol{c}}$; this is the bound $\overline{\boldsymbol{c}}=\boldsymbol{c}^{*}$, where the elements of the vector $\boldsymbol{c}^{*}$ are defined in (2.30). It then follows from Cavalcante et al. (2016, Proposition 1 and Facts 4.1 and 4.2) that the mapping $\boldsymbol{F}(\cdot)$ has a unique fixed point. This means that the system of equations (2.35) has a unique solution $\boldsymbol{c}(0)$.

\section{B.6.2 Properties of the solution: monotonicity}

For any vector $\boldsymbol{\delta}>>\mathbf{0}$, let this unique fixed point be denoted $\overline{\boldsymbol{c}}(\boldsymbol{\delta}$ ). (Note that the mapping $\boldsymbol{F}(\cdot)$ depends on the vector $\boldsymbol{\delta}$.) One can easily establish several features of the functional dependence of the fixed point on $\boldsymbol{\delta}$. In addition to being useful in the proof of Proposition 4 , these will be used in our later analysis of the effects of fiscal policy and credit policy.

First, because $\mathbf{0}<<\boldsymbol{F}(\boldsymbol{c}) \leq \boldsymbol{c}^{*}$ for all $\boldsymbol{c}$, it is clear that the fixed point must satisfy $0<<\overline{\boldsymbol{c}}(\boldsymbol{\delta}) \leq \boldsymbol{c}^{*}$ for all $\boldsymbol{\delta} \geq \mathbf{0}$.

We can also show that each of the component functions $\overline{\boldsymbol{c}}_{j}(\boldsymbol{\delta})$ must be at least weakly increasing in each of the elements of $\boldsymbol{\delta}$. Consider any two vectors $\boldsymbol{\delta}_{1}, \boldsymbol{\delta}_{2}$ such that $\boldsymbol{\delta}_{2} \geq \boldsymbol{\delta}_{1}>>$ 0. Then we can show that we must have $\overline{\boldsymbol{c}}\left(\boldsymbol{\delta}_{2}\right) \geq \overline{\boldsymbol{c}}\left(\boldsymbol{\delta}_{1}\right)$ for each $j$. Let $\boldsymbol{F}_{i}(\cdot)$ be the mapping defined by the right-hand side of (2.35) when $\boldsymbol{\delta}=\boldsymbol{\delta}_{i}$, for $i=1,2$, and further define the mapping

$$
\tilde{\boldsymbol{F}}(\boldsymbol{\zeta}) \equiv \boldsymbol{F}_{2}\left(\overline{\boldsymbol{c}}\left(\boldsymbol{\delta}_{1}\right)+\boldsymbol{\zeta}\right)-\overline{\boldsymbol{c}}\left(\boldsymbol{\delta}_{1}\right)
$$

defined for an arbitrary vector $\boldsymbol{\zeta} \geq \mathbf{0}$. Then $\boldsymbol{c}$ will be a fixed point of $\boldsymbol{F}_{2}$ if and only if $\boldsymbol{\zeta}=\boldsymbol{c}-\overline{\boldsymbol{c}}\left(\boldsymbol{\delta}_{1}\right)$ is a fixed point of $\tilde{\boldsymbol{F}}$.

It is evident that $\tilde{\boldsymbol{F}}(\cdot)$ is a continuous mapping, with the upper bound $\tilde{\boldsymbol{F}}(\boldsymbol{\zeta}) \leq \boldsymbol{c}^{*}-\overline{\boldsymbol{c}}\left(\boldsymbol{\delta}_{1}\right)$ for all $\boldsymbol{\zeta}$. Moreover, for any $\boldsymbol{\zeta} \geq \mathbf{0}$, we must have

$$
\begin{aligned}
\tilde{\boldsymbol{F}}(\boldsymbol{\zeta}) & \geq \boldsymbol{F}_{2}\left(\overline{\boldsymbol{c}}\left(\boldsymbol{\delta}_{1}\right)\right)-\overline{\boldsymbol{c}}\left(\boldsymbol{\delta}_{1}\right) \\
& \geq \boldsymbol{F}_{1}\left(\overline{\boldsymbol{c}}\left(\boldsymbol{\delta}_{1}\right)\right)-\overline{\boldsymbol{c}}\left(\boldsymbol{\delta}_{1}\right)=\mathbf{0}
\end{aligned}
$$


Thus $\tilde{\boldsymbol{F}}(\cdot)$ maps the set of vectors satisfying the bounds

$$
0 \leq \zeta \leq c^{*}-\bar{c}\left(\delta_{1}\right)
$$

into itself. These bounds define a compact, convex subset of $\mathbb{R}^{N}$. Hence by Brouwer's fixedpoint theorem, there must be a vector $\boldsymbol{\zeta}^{*}$ in this set that is a fixed point of the mapping $\tilde{\boldsymbol{F}}(\cdot)$. It follows that $\boldsymbol{c}=\overline{\boldsymbol{c}}\left(\boldsymbol{\delta}_{1}\right)+\boldsymbol{\zeta}^{*}$ is a fixed point of $\boldsymbol{F}_{2}(\cdot)$, and since (as shown above) the latter mapping must have a unique fixed point, it follows that we must have

$$
\overline{\boldsymbol{c}}\left(\boldsymbol{\delta}_{2}\right)=\overline{\boldsymbol{c}}\left(\boldsymbol{\delta}_{1}\right)+\boldsymbol{\zeta}^{*} \geq \overline{\boldsymbol{c}}\left(\boldsymbol{\delta}_{1}\right)
$$

Hence each of the functions $\bar{c}_{j}(\boldsymbol{\delta})$ must be weakly increasing in each of the elements of $\boldsymbol{\delta}$, as asserted.

This means that along any continuous expansion path $\boldsymbol{\delta}(s)$ for the vector $\boldsymbol{\delta}$ specifying period-zero liquidity, where the real variable $s$ indexes distance along the expansion path [not time], if each element of $\boldsymbol{\delta}(s)$ is at least weakly increasing in $s$, then each element of $\overline{\boldsymbol{c}}(\boldsymbol{\delta}(s))$ will be weakly increasing in $s$ as well. Among other things, this means that along any such expansion path (representing situations that can be reached through progressively larger lump-sum transfers in period zero), if the borrowing constraint no longer constrains some sector $j$ for the level of transfers parameterized by $s$, then sector $j$ will not be borrowingconstrained for any vector of transfers corresponding to a point $s^{\prime}>s$ along the expansion path.

Thus for each sector, there will be a single point along the expansion path at which that sector shifts from being borrowing-constrained (for all levels of transfers below that point) to being unconstrained (for all levels of transfers beyond that point). This is illustrated for two different network structures in Figure 3 (where the labeled points $\left\{\hat{a}_{i}\right\}$ on the horizontal axis are levels of initial liquid assets at which another sector ceases to be borrowing-constrained). If we let $C$ be the subset of the sectors that are borrowing-constrained in the case of a particular vector of initial asset positions, then as one proceeds along any monotonic expansion path, the set $C$ remains the same except at a finite number of points, and at any point where $C$ changes, increasing $s$ can only result in the subtraction of elements from $C$.

The set of possible vectors $\boldsymbol{\delta}$ can thus be partitioned into regions corresponding to different subsets $C$ of borrowing-constrained sectors. We have already shown (in the proof of Proposition 3) that for all $\boldsymbol{\delta}(0)$ close enough to $\mathbf{0}$, the set of unconstrained sectors will be $U_{0}$, the set of sectors $j$ for which the maximum value is achieved in the problem on the right-hand side of (2.31); hence at such points the set of constrained sectors will be $C_{0}$, the complement of $U_{0}$. Because the set of constrained sectors can only shrink as a result of additional initial transfers (or further relaxations of borrowing constraints), it follows that for all $\boldsymbol{\delta}(0)>>\mathbf{0}, C$ must be an element of $\mathcal{C}$, the set of all subsets of $C_{0}$ (including the empty set $\emptyset$ as well as $C_{0}$ itself). 


\section{B.6.3 The local solution for a given subset $C$ of borrowing-constrained sectors}

If we know what the set $C$ is for a given vector $\boldsymbol{\delta}$, it is straightforward to compute the equilibrium expenditure vector $\overline{\boldsymbol{c}}(\boldsymbol{\delta})$ at that point. ${ }^{50}$ The solution vector $\boldsymbol{c}(0)$ must satisfy

$$
c^{j}(0)=\frac{\delta^{j}}{\bar{p}}+\sum_{k} A_{j k} c^{k}(0)
$$

for all $j \in C$, and

$$
c^{j}(0)=c^{* j}
$$

for all $j \notin C$. This is a system of linear equations to solve for $\boldsymbol{c}(0)$.

The first of these sets of equations can be written in vector form as

$$
\hat{\boldsymbol{c}}=\hat{\boldsymbol{\delta}}+\boldsymbol{A}_{C C} \hat{\boldsymbol{c}}+\boldsymbol{A}_{C U} \check{\boldsymbol{c}}^{*},
$$

where $\hat{\boldsymbol{c}}$ is the vector of elements of the solution $\boldsymbol{c}(0)$ corresponding to sectors $j \in C, ; \hat{\boldsymbol{\delta}}$ is the vector collecting the values of $\delta^{j} / \bar{p}$ for the sectors $j \in C$; $\check{\boldsymbol{c}}^{*}$ is the vector of elements of $\boldsymbol{c}^{*}$ corresponding to sectors $k \notin C$; and the matrix $\boldsymbol{A}$ has been partitioned as in (3.2) in the main text. This system of linear equations has a unique solution (and hence the complete system has a unique solution) if and only if the matrix $\boldsymbol{I}-\boldsymbol{A}_{C C}$ is non-singular.

This is necessarily the case for any $C \in \mathcal{C}$. Note that in order for $\boldsymbol{I}-\boldsymbol{A}_{C C}$ to be singular, there would have to exist a vector $\boldsymbol{u} \neq \mathbf{0}$ such that $\boldsymbol{A}_{C C} \boldsymbol{u}=\boldsymbol{u}$. This would require that the set of sectors $C$ be an isolated subsystem (that is, sectors $j \in C$ spend only on the products of sectors $k \in C$ ). But we have shown in the proof of Proposition 3 that under our assumptions, the only isolated subsystem can be $S_{1}$, the one containing sector $N$. We have further shown that the eigenvector $\boldsymbol{\pi}$ has non-zero elements only for sectors $j \in S_{1}$. Hence the elements of $U_{0}$ (of which there must be at least one) belong to $S_{1}$; it follows that not all of $S_{1}$ can belong to $C_{0}$, and thus that not all of $S_{1}$ can belong to any $C \in \mathcal{C}$. We can therefore conclude that $C$ cannot be an isolated subsystem, from which it follows that $\boldsymbol{I}-\boldsymbol{A}_{C C}$ must be non-singular.

We can show something stronger, which is that all eigenvalues of $\boldsymbol{A}_{C C}$ must be inside the unit circle (i.e., have modulus less than 1). We note that $\boldsymbol{A}_{C C}$ is a non-negative matrix, though no longer a stochastic matrix (because the set $C$ cannot be an isolated subsystem, as just discussed). It follows from Gantmacher (1959, chap. XIII, Theorem 3) that $\boldsymbol{A}_{C C}$ has a non-negative real eigenvalue $r$, such that $|\lambda| \leq r$ for all of the other eigenvalues of the matrix. This maximal eigenvalue is bounded above by

$$
r \leq \max _{j \in C} \sum_{k \in C} A_{k j} \leq 1
$$

\footnotetext{
${ }^{50}$ Note that it may be ambiguous whether to include a particular sector $j$ in the set $C$ or not, as in equilibrium the sector's income may be just enough to allow it to spend the optimal quantity $c^{* j}$, with end-of-period assets $b^{j}(0)=0$. In this case, it does not matter whether we consider the set $C$ to include the sector $j$ or not; the solution obtained for $\overline{\boldsymbol{c}}(\boldsymbol{\delta})$ will be the same in either case. In such a case, the value of $\delta$ lies on the boundary between two regions corresponding to different sets of constrained sectors; but since the function $\overline{\boldsymbol{c}}(\boldsymbol{\delta})$ is continuous at such boundaries, it does not matter to which region the boundary case is assigned. Note that if instead we wish to compute the effect of a change in $\boldsymbol{\delta}$, it will matter how we define the set of constrained sectors $C$; but in that case, the right answer will depend on the direction in which $\boldsymbol{\delta}$ is to be changed.
} 
Here the first inequality follows from Gantmacher (p. 68), and the second from the definition of the matrix $\boldsymbol{A}$.

However, we have just shown that 1 cannot be an eigenvector of $\boldsymbol{A}_{C C}$. Thus the maximal eigenvalue must satisfy $r<1$, from which it follows that $|\lambda|<1$ for every eigenvalue of $\boldsymbol{A}_{C C}$. This allows us to write

$$
\left(\boldsymbol{I}-\boldsymbol{A}_{C C}\right)^{-1}=\boldsymbol{I}+\boldsymbol{A}_{C C}+\left(\boldsymbol{A}_{C C}\right)^{2}+\left(\boldsymbol{A}_{C C}\right)^{3}+\ldots
$$

where the infinite sum must converge because the eigenvalues of $\boldsymbol{A}_{C C}$ have modulus less than 1. Since each of the terms on the right-hand side is a non-negative matrix, it follows that

$$
\left(\boldsymbol{I}-\boldsymbol{A}_{C C}\right)^{-1} \geq \mathbf{0} .
$$

The system of linear local equations therefore has a unique solution

$$
\boldsymbol{c}^{l o c}(\boldsymbol{\delta} ; C)=\left[\begin{array}{c}
\left(\boldsymbol{I}-\boldsymbol{A}_{C C}\right)^{-1}\left(\hat{\boldsymbol{\delta}}+\boldsymbol{A}_{C U} \check{\boldsymbol{c}}^{*}\right) \\
\check{\boldsymbol{c}}^{*}
\end{array}\right],
$$

where the solution is partitioned as in (3.2); this is the solution (2.37 referred to in the proposition. (See the proof of Lemma 6, below, for further details.) Here we have written the set $C$ as an argument of the function, because there is a separate function of this kind for each possible choice of $C \in \mathcal{C}$. We further see that for any $C$, the solution is a linear function of $\boldsymbol{\delta}$ and $\boldsymbol{c}^{*}$, and that the matrices of coefficients denoted $\boldsymbol{M}$ and $\boldsymbol{N}$ in (2.37) contain only non-negative elements, because of (B.13) and the fact that all elements of $\boldsymbol{A}$ are non-negative.

\section{B.6.4 The unique global solution}

We see then that if we can determine which set of sectors $C$ is the borrowing-constrained set in the case of any given vector of initial asset balances, we can determine the value of $\overline{\boldsymbol{c}}(\boldsymbol{\delta})$ at that point. We next show how to do this. Fixing the vector $\boldsymbol{\delta}$, let $\bar{C}$ be the set of constrained sectors in the solution to the "Keynesian cross" system (2.35), and let $C$ instead be any other element of $\mathcal{C}$. Then let

$$
\zeta \equiv \boldsymbol{c}^{l o c}(\boldsymbol{\delta} ; \bar{C})-\boldsymbol{c}^{l o c}(\boldsymbol{\delta} ; C)
$$

measure the difference between the linear solution under the assumption that sectors $\bar{C}$ are constrained and the linear solution under the assumption instead that $C$ is the set of constrained sectors. (Also, in what follows, let us write $\boldsymbol{c}^{\text {loc }}(\boldsymbol{\delta} ; C)$ simply as $\boldsymbol{c}$, and $\boldsymbol{c}^{l o c}(\boldsymbol{\delta} ; \bar{C})$ as $\overline{\boldsymbol{c}}$.)

In the case of any sector $j \in C$, we must have

$$
\begin{aligned}
& c^{j}=\hat{\delta}^{j}+\sum_{k} A_{j k} c^{k}, \\
& \bar{c}^{j} \leq \hat{\delta}^{j}+\sum_{k} A_{j k} \bar{c}^{k},
\end{aligned}
$$


where the second condition holds for all $j$ given that $\overline{\boldsymbol{c}}$ is a solution to (2.35). Subtracting the first equation from the second yields the implication

$$
\zeta^{j} \leq \sum_{k} A_{j k} \zeta^{k}
$$

for all $j \in C$. Instead, in the case of any sector $j \notin C$, we must have

$$
\begin{aligned}
& c^{j}=c^{* j}, \\
& \bar{c}^{j} \leq c^{* j},
\end{aligned}
$$

where again the second condition holds for all $j$ given that $\overline{\boldsymbol{c}}$ is a solution to (2.35). Subtracting the first equation from the second yields the implication

$$
\zeta^{j} \leq 0
$$

for all $j \notin C$.

Then if we let $\hat{\boldsymbol{\zeta}}$ be the vector of elements of $\boldsymbol{\zeta}$ corresponding to sectors $j \in C$, and $\check{\boldsymbol{\zeta}}$ the vector of elements corresponding to sectors $j \notin C$, we must have

$$
\hat{\boldsymbol{\zeta}} \leq \boldsymbol{A}_{C C} \hat{\boldsymbol{\zeta}}+\boldsymbol{A}_{C U} \check{\boldsymbol{\zeta}}, \quad \check{\boldsymbol{\zeta}} \leq \mathbf{0}
$$

If we let $\boldsymbol{u} \equiv\left(\boldsymbol{I}-\boldsymbol{A}_{C C}\right) \hat{\boldsymbol{\zeta}}$, then the first inequality implies that $\boldsymbol{u} \leq \mathbf{0}$, and hence that

$$
\hat{\boldsymbol{\zeta}}=\left(\boldsymbol{I}-\boldsymbol{A}_{C C}\right)^{-1} \boldsymbol{u} \leq \mathbf{0}
$$

using (B.13). This together with the second inequality implies that $\boldsymbol{\zeta} \leq 0$, and hence that

$$
\boldsymbol{c}^{l o c}(\boldsymbol{\delta} ; \bar{C}) \leq \boldsymbol{c}^{l o c}(\boldsymbol{\delta} ; C)
$$

The fact that (B.14) must hold for any $C \in \mathcal{C}$ then implies that $\bar{C}$ must be the selection of borrowing-constrained sectors that implies that

$$
\overline{\boldsymbol{c}}(\boldsymbol{\delta})=\min _{C \in \mathcal{C}} \boldsymbol{c}^{l o c}(\boldsymbol{\delta} ; C)
$$

That is, for any $\boldsymbol{\delta}>\boldsymbol{>} \mathbf{0}, \bar{C}$ must be one of the elements of $\mathcal{C}$ that solve the minimization problem on the right-hand side of (B.15). Since (B.15) must hold for arbitrary $\boldsymbol{\delta}$, this gives us a closed-form solution for the function $\overline{\boldsymbol{c}}(\boldsymbol{\delta})$ for all $\boldsymbol{\delta}>>\mathbf{0}$.

If for values of $\boldsymbol{\delta}$ on the boundary of the positive orthant we select as the relevant solution to (2.35) the vector $\boldsymbol{c}(0)$ that can be reached as the limit of a sequence of solutions $\boldsymbol{c}_{n} \rightarrow \boldsymbol{c}(0)$ corresponding to a non-increasing sequence of vectors $\boldsymbol{\delta}_{n} \rightarrow \boldsymbol{\delta}$ with $\boldsymbol{\delta}_{n}>>\mathbf{0}$ for each $n$, then also for these values of $\boldsymbol{\delta}$ the solution for $\boldsymbol{c}(0)$ will be the one given by (B.15). (This follows immediately from the fact that the functions defined in (B.15) are all continuous functions of $\boldsymbol{\delta}$.) Hence (B.15) is the desired solution for all $\boldsymbol{\delta} \geq \mathbf{0}$, as stated in equation (2.38) of the proposition. 


\section{B.7 Proof of Corollary 2}

The proof follows exactly the same lines as in the proof of Corollary 1 above. Even when we allow non-negligible values for the $\left\{a^{j}(0)\right\}$, in the limit as $\beta \rightarrow 1$, the Euler condition again reduces to the form (2.29), which implies the upper bound (2.30) on spending by each sector. This was the crucial result needed to establish Corollary 1 . In the case that the $\left\{a^{j}(0)\right\}$ are non-negligible, the equation for the level of spending if a sector is borrowing-constrained changes, but the equation for its level of spending if it is not borrowing-constrained is unchanged, and the previous conclusions continue to hold.

\section{B.8 Proof of Corollary 3}

The solution to the system (2.35) involves no binding borrowing constraint for any sector if and only if $c^{j}(0)=c^{* j}$ for each $j$ is a solution to this system. Since we have established that the solution must be unique, if this is a solution it must be the unique solution; so it suffices that we check whether this vector of expenditure levels satisfies all of the equations in the system (2.35).

Substituting the candidate solution for $\boldsymbol{c}(0)$ into (2.34), we see that this equation holds if and only if

$$
\frac{\delta^{j}}{\bar{p}}+\sum_{k} A_{j k} c^{* k} \geq c^{* j} .
$$

Using (2.30) to substitute for the elements of $\boldsymbol{c}^{*}$ in this inequality, and recalling the definition (1.5), we obtain the requirement

$$
\frac{\delta^{j}}{\bar{p}}+\phi_{j}(0) \bar{y} \geq \omega_{j} \bar{y}
$$

This implies a lower bound for $\delta^{j}$ that is equivalent to condition (2.39). Hence the candidate solution satisfies (2.34) for all $j$ if and only if $\delta^{j}$ satisfies the lower bound (2.39) for all $j$. Hence (2.39) holding for all $j$ is necessary and sufficient for the solution to the system (2.35) to involve no binding borrowing constraints, as asserted in the corollary.

We have already noted that if borrowing constraints do not bind, consumption demands must be given by (2.32) for each sector. Then (2.28) implies that equilibrium production in each sector $k$ must equal

$$
y_{k}(0)=\sum_{j} c_{k}^{j}(0) \sum_{j} \alpha_{k-j} \phi_{k}(0) \bar{y}=\phi_{k}(0) \cdot \bar{y} .
$$

This establishes that the resource allocation must be (2.40).

Finally, in the special case that $a^{j}(0)=a(0) / N$ and $\underline{b}^{j}(0)=0$ for all $j$, we have $\delta^{j}=$ $a(0) / N$ for all $j$. In this case, (2.39) reduces to

$$
a(0) \geq N \bar{p} \bar{y} \cdot\left[\sum_{k} \alpha_{k-j} \phi_{k}(0)-\phi_{j}(0)\right] .
$$

This condition holds for all $j$ if and only if $a(0)$ satisfies the lower bound (2.41), as stated in the corollary. 


\section{Equilibrium with Fiscal Transfers}

Here we present proofs of the main results in section 3 of the main text.

\section{C.1 Proof of Lemma 5}

In any equilibrium, regardless of the nature of policy, the consumption allocation in period zero must satisfy (1.4). We wish to consider the conditions under which this can be consistent with achievement of the first-best allocation of resources, characterized in Lemma 2.

Consistency of (1.4) with (1.9) requires that for each pair of sectors $j, k$ such that $\alpha_{k-j} \phi_{k}(0)>0$ (so that $j$ wishes to consume good $k$ in period zero), one must have

$$
\frac{c^{j}(0)}{\omega_{j}}=\frac{y_{k}(0)}{\phi_{k}(0)} .
$$

(Note that $c_{k}^{j}(0) /\left(\alpha_{k-j} \phi_{k}(0)\right)$ must equal both of these quantities, so that they must be equal.) This means that for each of the goods $k$ consumed by sector $j$, the value of $y_{k}(0) / \phi_{k}(0)$ must be the same (and positive). But condition (1.8) implies that for each sector $k$,

$$
u^{\prime}\left(y_{k}(0) / \phi_{k}(0) ; \xi_{0}\right)=v^{\prime}\left(y_{k}(0) ; \xi_{0}\right)
$$

Hence $v^{\prime}\left(y_{k}(0) ; \xi_{0}\right)$ must be the same positive quantity for each of the sectors $k$ consumed by sector $j$.

Since this conclusion is independent of the identity of sector $j$, we can show more generally that $v^{\prime}\left(y_{k}(0) ; \xi_{0}\right)$ must be the same positive quantity for any two sectors that both sell a positive amount to some single sector. Our assumptions about the network structure further imply that the sector of sectors with $\phi_{k}(0)>0$ (and that are therefore consumed by someone) form an indecomposable system. It follows that $v^{\prime}\left(y_{k}(0) ; \xi_{0}\right)$ must be the same positive quantity for all $k$ such that $\phi_{k}(0)>0 .{ }^{51}$

If $v(y ; \xi)$ is strictly convex, as assumed in the lemma, then $v^{\prime}\left(y ; \xi_{0}\right)$ is an increasing function of $y$, and the conclusion of the previous paragraph is only possible if $y_{k}(0)$ is the same quantity for all $k$ such that $\phi_{k}(0)>0$. Moreover, the Inada condition on the function $u\left(c ; \xi_{0}\right)$ implies that (C.2) cannot have $y_{k}(0)=0$ as a solution, for any $\phi_{k}(0)>0$. Hence the common value for $y_{k}(0)$ for all of the sectors that produce must be positive.

Condition (C.2) further implies that if $y_{k}(0)$ is the same for every sector that produces, $y_{k}(0) / \phi_{k}(0)$ must also be the same for each of these sectors. And since the common value of $y_{k}(0)$ is positive, this is only possible if $\phi_{k}(0)$ is the same for each of the sectors with $\phi_{k}(0)>0$. This establishes the lemma.

Note that if instead $v\left(y ; \xi_{0}\right)$ is a linear function of $y$ (one of the cases considered in Proposition 5$), v^{\prime}\left(y ; \xi_{0}\right)$ will be the same for all $y>0$. Hence in this case, the conclusion reached above does not follow: it is possible to have different levels of production $y_{k}(0)$ in different sectors that all produce in period zero, and still achieve the first-best allocation of resources. In this case, we do not need to assume that $\phi_{k}(0)$ is the same for each of

\footnotetext{
${ }^{51}$ Note however that the value of $v^{\prime}\left(y_{k}(0) ; \xi_{0}\right)$ can be different for a sector with $\phi_{k}(0)=0$, if one exists, since in that case the ratio $c_{k}^{j}(0) /\left(\alpha_{k-j} \phi_{k}(0)\right)$ is undefined, and (C.1) need not be satisfied for this value of $k$.
} 
the sectors with $\phi_{k}(0)>0$ in order for the first-best allocation to be achievable under an appropriate policy, as Proposition 5 shows.

\section{C.2 Proof of Proposition 5}

We shall show that the equilibrium allocation of resources under this policy is given by

$$
c_{k}^{j}(t)=\alpha_{k-j} \phi_{k}(t) \bar{y}, \quad y_{k}(t)=\phi_{k}(0) \bar{y}
$$

for any $j, k$, and any period $t \geq 0$. The associated equilibrium prices are $p_{k}(t)=P^{*}(t)$ for each $k$ in any period $t \geq 0$, and the nominal interest rate $i(t)$ is given by (2.25) in each period $t \geq 0$. The associated path of beginning-of-period nominal asset balances is given by $a^{j}(t)=a(t) / N$ for each sector $j$ in any period $t \geq 1$, while end-of-period asset balances are given by $b^{j}(t)=a(t) / N$ for each sector in any period $t \geq 0$.

Note that if this is indeed an equilibrium under the proposed policy, (2.40) holds in period zero, as asserted in the proposition; equilibrium prices and quantities in all periods $t \geq 1$ are as specified in Proposition 1; and borrowing constraints do not bind in any sector in any period, since

$$
b^{j}(t)=a(t) / N>0 \geq \underline{b}^{j}(t)
$$

for any sector $j$ in any period $t \geq 0$.

Thus if the conjectured allocation is indeed an equilibrium, it remains only to show that this allocation of resources is the first-best optimal allocation defined in Lemma 2. It is easily verified that the proposed allocation satisfies (1.9) for all $j, k$, and $t$. In addition, the proposed output levels in any period $t \geq 1$ are $y_{k}(t)=\bar{y}$ for all $k$, and these satisfy (1.8) for each $k$ and any $t \geq 1$. When $t=0$, the proposed allocation specifies that $y_{k}(0)=0$ for any sector with $\phi_{k}(0)=0$, as is required by Lemma 2 . Thus we need only verify that the proposed output levels in each of the sectors with $\phi_{k}(0)>0$ also satisfy (1.8) in period $t=0$.

Under case (i) of the hypothesis, this condition becomes

$$
u^{\prime}\left(y_{k}(0) / \phi_{k}(0) ; \bar{\xi}\right)=\nu
$$

which is satisfied by the proposed allocation for each $k$ such that $\phi_{k}(0)>0$, since in the case of these preferences, $u^{\prime}(\bar{y} ; \bar{\xi})=\nu$. Under case (ii) of the hypothesis, the condition becomes

$$
u^{\prime}(\bar{y} ; \bar{\xi})=v^{\prime}(\bar{y} ; \bar{\xi})
$$

for every sector $k$ with $\phi_{k}(0)=1$, and this condition is satisfied by the definition of $\bar{\xi}$. Thus the proposed allocation satisfies all of the conditions stated in Lemma 2 for the first-best optimal allocation.

We then need only to show that the quantities and prices proposed in the first paragraph above do indeed constitute a perfect foresight equilibrium, under the policy specified in the proposition. This can be established using the same method as in the proof of Proposition 1. Because the proof is straightforward (following the proof strategy already illustrated in the earlier proof), we omit the details. We confine ourselves here to a sketch of the intuition for the result. First, the proposed end-of-period balances $\left\{b^{j}(0)\right\}$ imply that $\tilde{a}^{j}(1)$ is the same for each sector $j$, so that (2.24) implies that $\boldsymbol{f}=\mathbf{0}$ under the conjectured paths. And we have 
already shown in Lemma 3 that if $\boldsymbol{f}=\mathbf{0}$, the prices and quantities specified above represent a perfect foresight equilibrium from period $t=1$ onward. Second, an equilibrium in which $\boldsymbol{f}=\mathbf{0}$ going into period $t=1$ must have the equilibrium allocations described in Proposition 4 , even if $\beta<1$; the assumption that $\beta \rightarrow 1$ was only used in the proof of that proposition to guarantee that $\boldsymbol{f}=\mathbf{0}$. Third, the initial asset balances $\left\{a^{j}(0)\right\}$ specified in (3.1) imply that conditions (2.39) are satisfied for all sectors, in the case of any borrowing limits $\underline{b}^{j}(0) \leq 0$. Hence the same argument as is used in the proof of Corollary 3 can again be used to show that no sectors are borrowing-constrained in period zero, and that the equilibrium allocation of resources in period zero must be given by (2.32). And finally, the initial balances together with this pattern of spending and production in period zero imply the specified end-of-period balances $\left\{b^{j}(0)\right\}$, and hence that $\boldsymbol{f}=\mathbf{0}$. Thus we obtain an equilibrium of the conjectured form.

\section{C.3 Proof of Lemma 6}

Let $C \in \mathcal{C}$ be the set of constrained sectors in the case of initial liquidity $\boldsymbol{\delta}$. (Because our definition specifies that $c^{j}(0)$ is strictly less than $c^{* j}$ for each of the constrained sectors, these will continue to be the constrained sectors in the case of any small enough increase in the vector of initial assets.) Then we know, for each sector $j$, which of the two terms on the right-hand side of $(2.34) c^{j}(0)$ is equal to; this allows us to replace the nonlinear equation system (2.35) by a system of linear equations, that must hold locally at $\boldsymbol{\delta}$ and for any alternative vector $\boldsymbol{\delta}^{\prime} \geq \boldsymbol{\delta}$ close enough to it.

If we order the sectors so that all of the sectors in $C$ (if any) come first, and partition the matrix $\boldsymbol{A}$ as in (3.2), then the local version of (2.35) can be written as

$$
\left[\begin{array}{c}
\hat{\boldsymbol{c}} \\
\check{\boldsymbol{c}}
\end{array}\right]=\left[\begin{array}{l}
\hat{\boldsymbol{\delta}} \\
\check{\boldsymbol{\delta}}
\end{array}\right]+\left[\begin{array}{cc}
\boldsymbol{A}_{C C} & \boldsymbol{A}_{C U} \\
\mathbf{0} & \mathbf{0}
\end{array}\right]\left[\begin{array}{c}
\hat{\boldsymbol{c}} \\
\check{\boldsymbol{c}}
\end{array}\right]+\left[\begin{array}{ll}
\mathbf{0} & \mathbf{0} \\
\mathbf{0} & \boldsymbol{I}
\end{array}\right]\left[\begin{array}{c}
\hat{\boldsymbol{c}}^{*} \\
\check{c}^{*}
\end{array}\right]
$$

Here $\hat{\boldsymbol{c}}$ is the vector of expenditures $c^{j}(0)$ for the sectors $j \in C, \check{\boldsymbol{c}}$ is the vector of expenditures for sectors $j \notin C$; the vectors $\hat{\boldsymbol{\delta}}$ and $\check{\boldsymbol{\delta}}$ similarly collect the values of $\delta^{j} / \bar{p}$ for the two groups of sectors; and the vectors $\hat{\boldsymbol{c}}^{*}$ and $\check{\boldsymbol{c}}^{*}$ collect the values of $c^{* j}$ for the two groups of sectors.

For any $C \in \mathcal{C}$, we have already shown in the proof of Proposition 4 that $\boldsymbol{A}_{C C}$ has all of its eigenvalues inside the unit circle. It then follows that the matrix $\boldsymbol{I}-\boldsymbol{A}_{C C}$ must be invertible, and that its inverse can be expressed as the infinite sum on the right-hand side of (3.3). Hence the linear local system of equations has a unique solution of the form (2.37), where the matrices $\boldsymbol{M}$ and $\boldsymbol{N}$ are the ones given in the statement of the lemma. Moreover, the sub-matrix $\boldsymbol{M}_{C C}$ can alternatively be written as in (3.3).

Since $\boldsymbol{A}_{C C} \geq \mathbf{0}$, it follows from (3.3) that $\boldsymbol{M}_{C C} \geq \mathbf{0}$ as well. This together with the fact that $\boldsymbol{A}_{C U} \geq \mathbf{0}$ implies that all elements of the matrices $\boldsymbol{M}$ and $\boldsymbol{N}$ must be non-negative.

\section{C.4 Fiscal transfer multipliers: An example}

Our model implies that the multiplier effects of fiscal transfers can be quite different, depending both on the sectors receiving the transfer and which sectors' expenditure we are concerned with. As an example, consider again the chain network with fraction $\lambda$ of 
out-of-sector purchases (right panel of Figure 1), and consider the case of a pandemic shock which makes it impossible to consume the output of sector 1, while other sectors' products are unaffected. Suppose that we compute the multipliers for small additional transfers, starting from liquid asset balances that satisfy the inequality

$$
\sum_{j \neq N} a^{j}(0)<\lambda \bar{p} \bar{y}
$$

When this inequality is satisfied, sector $N$ is the only unconstrained sector (as already established in section $\mathrm{xx}$, for the limiting case in which $a(0) \rightarrow 0$ ), so that the set $C$ consists of sectors $\{1,2, \ldots, N-1\}$. The matrix of transfer multipliers is in this case equal to

$$
\boldsymbol{M}=\left[\begin{array}{cccccc}
1 & 0 & 0 & \ldots & 0 & 0 \\
\lambda^{-1} & \lambda^{-1} & 0 & \ldots & 0 & 0 \\
\lambda^{-1} & \lambda^{-1} & \lambda^{-1} & \ldots & 0 & 0 \\
\ldots & \ldots & \ldots & \ldots & \ldots & \ldots \\
\lambda^{-1} & \lambda^{-1} & \lambda^{-1} & \ldots & \lambda^{-1} & 0 \\
0 & 0 & 0 & \ldots & 0 & 0
\end{array}\right]
$$

The aggregate expenditure multiplier for transfers to sector $k$ can be obtained by summing column $k$ of the matrix $\boldsymbol{M}$; this is largest (equal to $1+(N-2) / \lambda$ ) for transfers to sector 1 , and smallest (zero) for transfers to sector $N$. In the numerical example discussed in the paper, $N=5$ and $\lambda=4 / 5$. In this case, the aggregate expenditure multiplier for a transfer to sector 1 is

$$
1+\lambda^{-1}+\lambda^{-1}+\lambda^{-1}+0=4.75
$$

as reported in the main text. The multiplier effect of uniformly distributed transfers on sector $j$ spending can be obtained by averaging the elements of row $j$ of the matrix; these are largest (equal to $(N-1) /(\lambda N))$ for sector $N-1$, and smallest (again zero) for sector $N$. In the numerical example discussed in the paper, the aggregate expenditure multiplier for uniformly distributed transfers is the average over the five columns of the sums of all five rows, or

$$
\frac{1}{5}[4.75+3.75+2.50+1.25+0.00]=2.45,
$$

as reported in the main text.

In the event that fewer sectors are borrowing-constrained, additional rows and columns of $\boldsymbol{M}$ must be set to zero. We continue to have a matrix of the form

$$
\boldsymbol{M}=\left[\begin{array}{cccccc}
1 & 0 & 0 & \ldots & 0 & 0 \\
\lambda^{-1} & \lambda^{-1} & 0 & \ldots & 0 & 0 \\
\lambda^{-1} & \lambda^{-1} & \lambda^{-1} & \ldots & 0 & 0 \\
\ldots & \ldots & \ldots & \ldots & \ldots & \ldots \\
0 & 0 & 0 & \ldots & 0 & 0
\end{array}\right],
$$

but if $\hat{a}_{i-1} \leq a(0)<\hat{a}_{i}$, for any $i \geq 1$, the last $i$ rows of the matrix are all zeroes. Thus when $i=1$, as assumed above, the aggregate expenditure multiplier for uniformly distributed transfers is given by (C.4). But if $i=2$, it is instead equal to only

$$
\frac{1}{5}[3.50+2.50+1.25+0.00+0.00]=1.45
$$


if $i=3$, it is equal to only

$$
\frac{1}{5}[2.25+1.25+0.00+0.00+0.00]=0.70
$$

and if $i=4$, it is equal to only

$$
\frac{1}{5}[1.00+0.00+0.00+0.00+0.00]=0.20 \text {. }
$$

And of course, once $a(0) \geq \hat{a}_{4}$, the entire matrix of multipliers is equal to zero.

The differentiation of alternative multipliers according to the sector affected (the separate rows of $\boldsymbol{M}$ ) is relevant for calculation of the welfare effects of transfer policies, since the marginal utility of additional spending varies across sectors. For example, when (C.3) holds, the marginal utility of additional real expenditure by any sector $j \neq N$ is given by

$$
\mu^{j} \equiv u^{\prime}\left(c^{j}(0) /\left(1-\alpha_{1-j}\right)=u^{\prime}\left(\lambda^{-1} \sum_{k=1}^{j} a^{k}(0)\right),\right.
$$

while for sector $N$ it is

$$
\mu^{N} \equiv u^{\prime}\left(c^{N}(0) /\left(1-\alpha_{1}\right)\right)=u^{\prime}(\bar{y})
$$

It follows that in the case of any $\boldsymbol{a}(0)>>\mathbf{0}$ satisfying (C.3),

$$
\mu^{1}>\mu^{2} \ldots \mu^{N-1}>\mu^{N} .
$$

The welfare effect of transfers to each of the different sectors is then given not by $\boldsymbol{e}^{\prime} \boldsymbol{M}$ (the vector of column sums), but by $\boldsymbol{\mu}^{\prime} \boldsymbol{M}$, where $\boldsymbol{\mu}$ is the $N$-vector with $j$ th element equal to $\mu^{j}$.

\section{C.5 Proof of Corollary 4}

Given a one-parameter family of transfer policies (3.4), let $c^{j}(s)$ be the solution for $c^{j}(0)$ in the case of the transfer policy indexed by $s$. It follows from (2.38) that

$$
c^{j}(s)=\min _{C \in \mathcal{C}} c^{j, l o c}(\boldsymbol{\alpha}+\boldsymbol{\gamma} \cdot s ; C) .
$$

It follows from Lemma 6 that each of the functions $c^{j, l o c}(\boldsymbol{\alpha}+\boldsymbol{\gamma} \cdot s ; C)$ is a non-increasing linear function of $s$. Since $c^{j}(s)$ is the minimum of a finite collection of such functions, it must be a non-increasing, piecewise-linear, concave function. This then implies that its right derivative, $m_{j}(s)$, must a piecewise constant function, non-increasing in $s$, and everywhere non-negative in value.

It remains only to show that $m_{j}(s)$ is eventually equal to zero for all $s$ above some finite bound. But since $c^{j}(s)$ can have only a finite number of segments with different slopes, there must be some finite $\bar{s}$ such that $m_{j}(s)$ is constant for all $s \geq \bar{s}$. Suppose that this terminal value is positive. It would follow that $c^{j}(s)$ would be an increasing linear function of $s$ for all $s \geq \bar{s}$. But it follows from (2.30) that $c^{j}(s)$ must be bounded. Hence we obtain a contradiction, and can conclude that instead $m_{j}(s)$ must equal zero for all $s \geq \bar{s}$, as asserted in the corollary. 


\section{C.6 Proof of Lemma 7}

For any choice of the parameters specifying policy in period 1 , and any value of $\beta$ close enough to 1, there must be an equilibrium of the following kind. First, the equilibrium for all $t \geq 1$ is a stationary equilibrium of the kind characterized in Lemma 3, for some vector $\boldsymbol{f} \in U$. (The stipulation that $\beta$ be close enough to 1 is in order to ensure that $\boldsymbol{f} \in U$.) Second, for each sector $j$, the consumption plan in period $t=0$ must be of the form (1.4) for some choice of $c^{j}(0)$. This allows us to compute end-of-period balances $b^{j}(0)$ as a function of $c^{j}(0)$, so that constraint (1.12) implies an upper bound for $c^{j}(0)$, that depends on the policy specification (both on $a^{j}(0)$ and on $\left.\underline{b}^{j}(0)\right)$. Total expenditure $c^{j}(0)$ must also satisfy

$$
u^{\prime}\left(c^{j}(0) / \omega_{j} ; \bar{\xi}\right) \geq \psi \Lambda^{* j}(\boldsymbol{f}),
$$

as a consequence of (2.27). This also implies an upper bound for $c^{j}(0)$ that depends on policy (in particular, that depends on the monetary policy parameter $\psi$ ). Since at least one of the constraints (1.12) and (C.5) must hold with equality, the optimal choice of $c^{j}(0)$ must be the minimum of these two upper bounds, for each sector $j$. Third, the vector $\boldsymbol{f}$ must satisfy (2.24), where

$$
\tilde{a}^{j}(1)=\psi \frac{b^{j}(0)}{\bar{p}}
$$

for each sector $j$, and we can compute $b^{j}(0)$ from the sector's choice of $c^{j}(0)$, as just indicated. This gives us a fixed-point relationship, $\boldsymbol{f}=\boldsymbol{\Psi}(\boldsymbol{f})$, that the vector $\boldsymbol{f}$ must satisfy. The mapping $\boldsymbol{\Psi}$ is defined for any $\boldsymbol{f} \in U$.

In the limiting case $\beta=1$, this fixed-point relationship becomes a mapping $\Psi(\boldsymbol{f})=\mathbf{0}$ for all $\boldsymbol{f}$, and there exists a unique fixed point, $\boldsymbol{f}^{*}=\mathbf{0}$. For any values of $\beta$ close enough to 1 , the mapping will still be a contraction, and will have a unique fixed point $\boldsymbol{f}^{*}$ near $\mathbf{0}$. Hence there is a perfect foresight equilibrium of the kind proposed above. Moreover, in the limit as $\beta \rightarrow 1$, the fixed point $\boldsymbol{f}^{*} \rightarrow \mathbf{0}$. Lemma 4 then implies that the stationary allocation in periods $t \geq 1$ approaches the one characterized in Proposition 1 (for the case in which $\xi_{t}=\bar{\xi}$ each period), which is to say, the stationary allocation that represents the first-best optimum when $\xi_{t}=\bar{\xi}$.

It then follows that $\bar{U}^{j} \rightarrow U^{*}$ in this limit, so that (3.6) must hold, as asserted in the lemma. This in turn implies that (3.7) provides an equivalent welfare ranking of alternative policies in this limit.

We also note that Lemma 4 implies that $\Lambda^{* j}(\boldsymbol{f}) \rightarrow u^{\prime}(\bar{y} ; \bar{\xi})$ in the limit as $\beta \rightarrow 1$, so that the upper bound implied by (C.5) approaches

$$
c^{j}(0) \leq c^{* j}(\psi) \equiv \psi \omega_{j} \bar{y}
$$

a generalization of (2.30). The vector $\boldsymbol{c}(0)$ of equilibrium spending levels in period zero is then the solution to the fixed-point system (2.35), given by (2.38), in which we now substitute the more general definition of $c^{* j}$ given in (C.6). This result is useful for characterizing the effects of interest-rate policy, discussed further below.

\section{C.7 Proof of Proposition 6}

Substitution of (1.2) into the welfare measure (3.7), and simplification using the fact that in any equilibrium the consumption plan for each sector must be of the form (1.4), allows us 
to write the welfare measure in the form

$$
W_{0}=\sum_{j=1}^{N} \omega_{j} G\left(c^{j}(0) / \omega_{j}\right)+\sum_{k=1}^{N} H\left(y_{k}(0)\right),
$$

where

$$
G(c) \equiv u(c ; \bar{\xi}))-u^{\prime}(\bar{y} ; \bar{\xi}) \cdot c, \quad H(y) \equiv v^{\prime}(\bar{y} ; \bar{\xi}) \cdot y-v(y ; \bar{\xi})
$$

We can then use our solutions for the effects of transfers on $c^{j}(0)$ and $y_{k}(0)$ to calculate the welfare gradient.

Differentiating (C.7) with respect to each element of the vector of transfers, we obtain equation (3.8) given in the main text, where $\boldsymbol{g}$ is the vector with elements

$$
g_{j}=G^{\prime}\left(c^{j}(0) / \omega_{j}\right)
$$

for each $j$, and $\boldsymbol{h}$ is the vector with elements

$$
h_{k}=H^{\prime}\left(y_{k}(0)\right)
$$

for each $k$. (We use that $\boldsymbol{M}$ is the matrix of expenditure multipliers and $\boldsymbol{M}^{Y}=\boldsymbol{A} \boldsymbol{M}$ is the matrix of output multipliers.) Differentiating the functions $G$ and $H$, we obtain the expressions for $g_{j}$ and $h_{k}$ given in the main text.

We further observe that $G(c)$ is a strictly concave function, that reaches its (unique) maximum at $c=\bar{y}$, and that $H(y)$ is another (at least weakly) concave function, which achieves its maximum value at $y=\bar{y}$ (though the maximum need not be unique). It follows that $g_{j}$ must be positive for all $c^{j}(0) / \omega_{j}<\bar{y}$ (or for all $c^{j}(0)<c^{* j} \equiv \omega_{j} \bar{y}$, as stated in the text), and similarly that $h_{k}$ must be non-negative for all $y_{k}(0) \leq \bar{y}$. Since the elements of $\boldsymbol{M}$ and $\boldsymbol{M}^{Y}$ are all non-negative, we obtain the result that the elements of the welfare gradient must all be non-negative if we consider additional transfers at a point where $c^{j}(0) \leq c^{* j}$ for all $j$ and $y_{k}(0) \leq \bar{y}$ for all $k$.

When $\psi=1$, the Euler condition (2.29) must hold. Then because of (2.30), we must have $c^{j}(0) \leq c^{* j}$ for all $j$ in all cases. This in turn implies that $c_{k}^{j}(0) \leq \alpha_{k-j} \phi_{k}(0) \bar{y}$ for all $j, k$, using (1.4), and hence that $y_{k}(0) \leq \phi_{k}(0) \bar{y}$ for all $k$, as a consequence of (1.14). (The reasoning is the same as in the derivation of (B.9). Under the further assumption that $0 \leq \phi_{k}(0) \leq 1$ for all sectors, we must have $y_{k}(0) \leq \bar{y}$ for all $k$, and all elements of the welfare gradient must be non-negative.

Now consider the effects of a transfer to sector $j$ only, meaning that the vector of transfers is proportional to $\boldsymbol{e}_{j}$, the vector with 1 as its $j$ th element, and all other elements equal to 0. If $j \notin C$ (sector $j$ is not borrowing-constrained), it follows from Lemma 6 that $\boldsymbol{M} \boldsymbol{e}_{j}=\mathbf{0}$, as a consequence of which $w_{j} \equiv \boldsymbol{w}^{\prime} \boldsymbol{e}_{j}=0$. If instead $j \in C$, it follows from (3.3) that $M_{j j}>0$, and hence that

$$
w_{j} \equiv \boldsymbol{w}^{\prime} \boldsymbol{e}_{j} \geq \boldsymbol{g}^{\prime} \boldsymbol{M} \boldsymbol{e}_{j} \geq g_{j} M_{j j}>0
$$

\section{C.8 Proof of Proposition 7}

The proof proceeds in the same way as in the proof of Proposition 5. In the earlier proof, the key to establishing existence of an equilibrium with the first-best optimal allocation of 
resources was demonstrating that the assumed policies were consistent with (i) an equilibrium for periods $t \geq 1$ of the kind characterized in Lemma 3 with $\boldsymbol{f}=\mathbf{0}$; and (ii) an equilibrium in period $t=0$ in which no sectors are borrowing-constrained, so that the equilibrium allocation of resources is given by (2.40). In the limit as $\beta \rightarrow 1$, we must have $\boldsymbol{f} \rightarrow \mathbf{0}$ regardless of the nature of equilibrium in period $t=0$, so that condition (i) is now more easily established.

Given an equilibrium for periods $t \geq 1$ with $\boldsymbol{f}=\mathbf{0}$, the Euler condition takes the form (2.29), which implies that the equilibrium in period zero must satisfy (2.30). It then follows, as in the proof of Corollary 3, that (2.39) is a sufficient condition for the existence of an equilibrium in period zero in which borrowing constraints do not bind in any sector. One can then establish that the equilibrium must involve the first-best optimal allocation of resources using the same argument as in the proof of Proposition 5.

\section{Equilibrium with Interest-Rate Policy}

Here we present proofs of the main results in section 4 of the main text.

\section{D.1 Proof of Proposition 8}

The proof follows exactly the same lines as the proof of Proposition 3, but taking into account the fact that (2.29) now takes the more general form (C.5). As before, the assumption that initial asset balances are negligible and that borrowing is impossible implies that the equilibrium for periods $t \geq 1$ must be of the kind characterized in Lemma 3 , for the case in which $\boldsymbol{f}=\mathbf{0}$. The equilibrium in period $t=0$ must be one in which $b^{j}(0) \rightarrow 0$ for each sector $j$, with the consequence that $\boldsymbol{c}(0)$ must be a multiple of the maximal eigenvector $\boldsymbol{\pi}$, as in Proposition 3. The fact that the inequality (4.2) must hold for each sector, and with equality for at least one sector, then implies that the multiplicative factor $\Omega$ must be given by (4.4).

\section{D.2 Proof of Corollary 5}

It follows from Proposition 8 that the allocation of resources in periods $t \geq 1$ is independent of the choice of $\psi$; hence (1.7) is maximized by the policy that maximizes the single-period welfare criterion (3.7). Using (1.2) and (1.4), we can write (3.7) in the form

$$
W_{0}=\sum_{j}\left[\omega_{j} u\left(\frac{c^{j}(0)}{\omega_{j}} ; \bar{\xi}\right)-v\left(y_{j}(0) ; \bar{\xi}\right)\right] \text {. }
$$

Here both the quantities $\left\{c^{j}(0)\right\}$ and $\left\{y_{j}(0)\right\}$ depend on $\psi$ purely through the effect of $\psi$ on the value of $\hat{y}(\psi)$. Hence we can reduce the problem of choosing $\psi$ to maximize (1.7) to the problem of choosing $\hat{y}$ to maximize (D.1). (The optimal $\psi$ will then be whatever value is required in order for $\hat{y}(\psi)$ to equal the optimal value of $\hat{y}$.)

Proposition 8 implies that each of the quantities $\left\{c^{j}(0)\right\}$ and $\left\{y_{j}(0)\right\}$ is a non-negative multiple of $\hat{y}(\psi)$; in addition, at least one of the $\left\{c^{j}(0)\right\}$ and at least one of the $\left\{y_{j}(0)\right\}$ are positive, and hence strictly increasing function of $\hat{y}$. It then follows from (D.1) that $W_{0}$ 
must be a strictly concave function of $\psi$, with a continuous derivative. Hence $W_{0}(\psi)$ must have a unique maximum, which must furthermore be the unique point consistent with the first-order condition that $\partial W_{0} / \partial \hat{y}$ be equal to zero.

Differentiating (D.1) with respect to $\hat{y}$, we obtain

$$
\begin{aligned}
\frac{\partial W_{0}}{\partial \hat{y}} & =\sum_{j}\left[u^{\prime}\left(\frac{c^{j}(0)}{\omega_{j}} ; \bar{\xi}\right) \frac{\partial c^{j}(0)}{\partial \hat{y}}-v^{\prime}\left(y_{j}(0) ; \bar{\xi}\right) \frac{\partial y_{j}(0)}{\partial \hat{y}}\right] \\
& =\sum_{j}\left[u^{\prime}\left(\frac{c^{j}(0)}{\omega_{j}} ; \bar{\xi}\right) \frac{\partial c^{j}(0)}{\partial \hat{y}}\right]-\nu(\bar{\xi}) \cdot \sum_{k} \frac{\partial y_{k}(0)}{\partial \hat{y}} \\
& =\sum_{j}\left\{\left[u^{\prime}\left(\frac{c^{j}(0)}{\omega_{j}} ; \bar{\xi}\right)-\nu(\bar{\xi}) \cdot \sum_{k} A_{k j}\right] \frac{\partial c^{j}(0)}{\partial \hat{y}}\right\} \\
& =\sum_{j}\left\{\left[u^{\prime}\left(\frac{c^{j}(0)}{\omega_{j}} ; \bar{\xi}\right)-\nu(\bar{\xi})\right] \frac{\partial c^{j}(0)}{\partial \hat{y}}\right\},
\end{aligned}
$$

using the assumed form for $v(y ; \xi)$.

The corollary assumes that each sector $j$ will belong either to a set of borrowingconstrained sectors $C$ with $c^{j}(0)=0$ (which requires that $\pi_{j}=0$ ), or to the complementary set of sectors $U$, for which (4.2) holds with equality. (Note that under the assumptions of this corollary, the set of borrowing-constrained sectors is the same for all values of $\psi$.) For any $j \in C$, the fact that $\pi_{j}=0$ means that $\partial c^{j}(0) / \partial \hat{y}=0$, using the solution for $c^{j}(0)$ in Proposition 8. Hence these terms contribute nothing to the sum in (D.2). Moreover, the fact that (4.2) holds with equality for every sector $j \in U$ allows us to write the remaining terms in the form

$$
\frac{\partial W_{0}}{\partial \hat{y}}=\left[u^{\prime}(\hat{y} ; \bar{\xi})-\nu(\bar{\xi})\right] \sum_{j \in U} \frac{\partial c^{j}(0)}{\partial \hat{y}} .
$$

The solution in Proposition 8 implies that $c^{j}(0)$ is a linearly increasing function of $\hat{y}$ for each of the sectors $j \in U$ (and there must be at least one such sector); hence the sum of the $\partial c^{j}(0) / \partial \hat{y}$ terms must be positive. Moreover, the fact that $u(c ; \bar{\xi})$ is strictly concave implies that $u^{\prime}(\hat{y} ; \bar{\xi})$ must be a decreasing function of $\hat{y}$. Therefore the expression in square brackets is positive if $\hat{y}<\bar{y}$ and negative if $\hat{y}>\bar{y}$. It follows that $\partial W_{0} \partial \hat{y}$ is positive for all $\hat{y}<\bar{y}$ and negative for all $\hat{y}>\bar{y}$.

From this we can conclude that $W_{0}$ is (uniquely) maximized when $\psi$ is chosen so that $\hat{y}(\psi)=\bar{y}$, which holds if and only if $\psi=1$ (the policy assumed in Proposition 3).

\section{D.3 Proof of Proposition 9}

The result that the equilibrium for periods $t \geq 1$ is the same as in Proposition 1 can be established in the same way as in the proof of Proposition 4, since it is once again the case that in the limit as $\beta \rightarrow 1$, we must have $\boldsymbol{f} \rightarrow \mathbf{0}$, regardless of the nature of the equilibrium allocation in period $t=0$.

The demonstration that the system of equations (4.5) has a unique solution for $\boldsymbol{c}(0)$ also proceeds in the same way as in the proof of Proposition 4. In fact, for any given specification 
of policy, the equations (and hence their solution) are exactly the same, except that the vector of parameters $\boldsymbol{c}^{*}>\boldsymbol{>} \mathbf{0}$ in the previous discussion is now replaced by the vector $\boldsymbol{\omega} \cdot \hat{y}(\psi)$, which indicates how the elements of the vector depend on the choice of $\psi$. (The more specific assumption that $\boldsymbol{c}^{*}=\boldsymbol{\omega} \cdot \bar{y}$ was never used in the derivation of (2.38), only the fact that the elements of the vector were all positive.) Hence we obtain a unique solution $\boldsymbol{c}(\boldsymbol{\delta} ; \psi)$, given by our previous solution (2.38), but with the above substitution for the vector $c^{*}$.

Finally, the fact that the right-hand side of (4.5) is homogeneous of degree one in $(\boldsymbol{c}(0), \boldsymbol{\delta}, \hat{y})$ implies that if some values $(\boldsymbol{c}(0), \boldsymbol{\delta}, \hat{y})$ satisfy the equation, the values $(\lambda \boldsymbol{c}(0), \lambda \boldsymbol{\delta}, \lambda \hat{y})$ must satisfy it as well, for any multiplicative factor $\lambda>0$. This means that if $\boldsymbol{c}(0)=\boldsymbol{c}$ is a solution to (4.5) in the case of a policy that implies parameters $(\boldsymbol{\delta}, \hat{y})$, then $\boldsymbol{c}(0)=\lambda \boldsymbol{c}$ will be a solution in the case of a policy that implies parameters $(\lambda \boldsymbol{\delta}, \lambda \hat{y})$. Thus the function $\boldsymbol{c}(\boldsymbol{\delta} ; \psi)$ must be a homogeneous degree one function of $(\boldsymbol{\delta}, \hat{y}(\psi))$.

From this it follows that the function can be written in the form

$$
\boldsymbol{c}(\boldsymbol{\delta} ; \psi)=\frac{\hat{y}(\psi)}{\bar{y}} \cdot \overline{\boldsymbol{c}}\left(\frac{\bar{y}}{\hat{y}(\psi)} \boldsymbol{\delta}\right),
$$

where

$$
\overline{\boldsymbol{c}}(\boldsymbol{\delta}) \equiv \boldsymbol{c}(\boldsymbol{\delta} ; 1)
$$

Moreover, when $\psi=1$, the system (4.5) reduces to (2.35), the system for which we have already determined the solution. Hence the function $\overline{\boldsymbol{c}}(\boldsymbol{\delta})$ must be the one defined in (2.38).

Given this solution for the sectoral expenditure levels, the fact that prices are predetermined the level $p_{k}(0)=\bar{p}$ for all $k$ implies that the complete allocation of resources in period zero is given by (1.4) and (1.14).

\section{D.4 Proof of Corollary 6}

It follows from the form of the solution for $\boldsymbol{c}(0)$ in Proposition 9, together with (2.38), that we can write

$$
\begin{aligned}
\boldsymbol{c}(0) & =\frac{\hat{y}(\psi)}{\bar{y}} \cdot \min _{C \in \mathcal{C}} \boldsymbol{c}^{l o c}\left(\frac{\bar{y}}{\hat{y}(\psi)} \boldsymbol{\delta} ; C\right) \\
& =\frac{\hat{y}(\psi)}{\bar{y}} \cdot \min _{C \in \mathcal{C}}\left\{\frac{\bar{y}}{\hat{y}(\psi)} \boldsymbol{M}(C) \boldsymbol{\delta}+\boldsymbol{N}(C) \boldsymbol{c}^{*}\right\} \\
& =\min _{C \in \mathcal{C}}\{\boldsymbol{M}(C) \boldsymbol{\delta}+\boldsymbol{N}(C) \boldsymbol{\omega} \cdot \hat{y}(\psi)\},
\end{aligned}
$$

where the notation $\boldsymbol{M}(C), \boldsymbol{N}(C)$ indicates that the matrices $\boldsymbol{M}$ and $\boldsymbol{N}$ in (2.37) depend on the choice of the set $C$. The expression on the final line indicates that each of the solutions $c^{j}(0)$ is the minimum of a finite collection of non-decreasing linear functions of $\hat{y}(\psi)$. It follows that $c^{j}(0)$ must be a non-decreasing, piecewise linear, concave function of $\hat{y}(\psi)$.

Since $y^{a g g}(0) \equiv \sum_{k} y_{k}(0)=\sum_{j} c^{j}(0)$, aggregate output is the sum of a finite collection of non-decreasing, piecewise linear, concave functions of $\hat{y}(\psi)$, and hence must itself be a nondecreasing, piecewise linear, concave function of $\hat{y}(\psi)$. Moreover, for every partition $C \in \mathcal{C}$, 
the sectors $j \in U_{0}$ (which must include at least one sector) are among the unconstrained sectors. It then follows from Lemma 6 that for any $j \in U_{0}$, the coefficient

$$
\boldsymbol{e}_{j}^{\prime} \boldsymbol{N}(C) \boldsymbol{\omega}=\omega_{j}>0
$$

for any $C \in \mathcal{C}$, so that $c^{j}(0)$ must be increasing as $\hat{y}$ increases, no matter how large $\hat{y}$ may be. Since at least one such sector exists, the sum $y^{a g g}(0)=\sum_{j} c^{j}(0)$ must be strictly increasing as well. Thus $y^{a g g}(0)$ is an increasing, piecewise linear, concave function of $\hat{y}(\psi)$, as asserted in the corollary.

Each of the quantities $c_{k}^{j}(0), y_{k}(0)$, for arbitrary $j, k$, is similarly a linear combination of the different elements of $\boldsymbol{c}(0)$ with non-negative weights; and so by the same kind of argument, we can show that each of these quantities is a non-decreasing, piecewise linear, concave function of $\hat{y}(\psi)$, as asserted in the corollary.

Let us next consider how the set $C$ of borrowing-constrained sectors changes as we lower $\psi$, increasing $\hat{y}(\psi)$. The system (4.5) implies that for each sector $j$,

$$
c^{j}(0)=\min \left\{L^{j}(\hat{y}(\psi) ; C(\psi)), \omega_{j} \hat{y}(\psi)\right\}
$$

where $C(\psi)$ is the set of borrowing-constrained sectors in the case of interest-rate policy $\psi$, and for each possible choice of $C, L^{j}(\hat{y} ; C)$ is the function

$$
L^{j}(\hat{y} ; C)=\frac{\delta^{j}}{\bar{p}}+\boldsymbol{e}_{j}^{\prime} \boldsymbol{A} \boldsymbol{c}^{l o c}(\hat{y} ; C),
$$

where we use the notation

$$
\boldsymbol{c}^{l o c}(\hat{y} ; C) \equiv \boldsymbol{M}(C) \boldsymbol{\delta}+\boldsymbol{N}(C) \boldsymbol{\omega} \cdot \hat{y}(\psi)
$$

for the functions of $\hat{y}$ on the right-hand side of (D.4) associated with the different possible choices of $C$.

Consider a value of $\psi$ at which a particular set of sectors $C(\psi)=C_{1}$ are borrowingconstrained. This means that for sectors $j \in C_{1}, L^{j}\left(\hat{y}(\psi) ; C_{1}\right)<\omega_{j} \hat{y}(\psi)$, while for the unconstrained sectors, $L^{j} \geq \omega_{j} \hat{y}$. And now consider whether the set $C(\psi)$ remains equal to $C_{1}$ if $\psi$ is reduced relative to this initial value. In order for this to be the case, it must continue to be true that $L^{j}\left(\hat{y}(\psi) ; C_{1}\right)<\omega_{j} \hat{y}(\psi)$ for sectors $j \in C_{1}$, while $L_{j} \geq \omega_{j} \hat{y}(\psi)$ continues to hold for all of the other sectors.

We see from (D.5) that for each $j, L^{j}\left(\hat{y}(\psi) ; C_{1}\right)$ is the sum of a non-negative constant plus a non-negative term proportional to $\hat{y}(\psi)$; it follows that $L^{j}\left(\hat{y}(\psi) ; C_{1}\right.$ either grows in proportion to the growth of $\hat{y}(\psi)$ (in the case that $\delta^{j}=0$ ), or less than proportionally to the growth of $\hat{y}(\psi)$ (in the case that $\delta^{j}>0$ ), when $\psi$ is reduced. In either case, the fact that $L^{j}\left(\hat{y}(\psi) ; C_{1}\right)<\omega_{j} \hat{y}(\psi)$ initially for sectors $j \in C_{1}$ means that this inequality must continue to hold for any smaller value of $\psi$, given that $\omega_{j} \hat{y}(\psi)$ grows in proportion to $\hat{y}(\psi)$. Hence it cannot be the case that any of the borrowing-constrained sectors ceases to be borrowingconstrained as a result of a reduction in $\psi$.

On the other hand, it is possible that a reduction in $\psi$ will eventually increase $\hat{y}(\psi)$ sufficiently for one of the initially unconstrained sectors to cease to be unconstrained. If so, at that point the set $C(\psi)$ must change; but the change must always be an increase in the 
set of borrowing-constrained sectors. The sectors $j \in C_{1}$ that were previously borrowingconstrained must continue to be constrained. Thus as $\psi$ is reduced, the set $C(\psi)$ can only be increasing, as asserted in the corollary.

Finally, we consider the behavior of the solution to the system (4.5) when the real interest rate is very low. We begin by noting that one of the functions $\boldsymbol{c}^{l o c}(\hat{y} ; C)$ on the right-hand side of (D.4) is the one associated with the partition $C=C_{0}$. In the case of this choice of the set of constrained sectors, the definition of the eigenvector $\boldsymbol{\pi}$ implies that

$$
\left(\boldsymbol{I}-\boldsymbol{A}_{C C}\right) \boldsymbol{\pi}_{C}=\boldsymbol{A}_{C U} \boldsymbol{\pi}_{U},
$$

and hence that

$$
\boldsymbol{\pi}=\left[\begin{array}{l}
N_{C U} \\
\boldsymbol{N}_{U U}
\end{array}\right] \boldsymbol{\pi}_{U}
$$

Moreover, for each of the sectors $j \in U_{0}$, it follows from (2.31) that

$$
\pi_{j}=\left[\max _{\ell}\left(\pi_{\ell} / \omega_{\ell}\right)\right] \cdot \omega_{j}
$$

hence we have

$$
\boldsymbol{\pi}_{U}=\left[\max _{\ell}\left(\pi_{\ell} / \omega_{\ell}\right)\right] \cdot \boldsymbol{\omega}_{U}
$$

Substitution of this into (D.6) yields

$$
\boldsymbol{\pi}=\left[\max _{\ell}\left(\pi_{\ell} / \omega_{\ell}\right)\right] \cdot\left[\begin{array}{c}
\boldsymbol{N}_{C U} \\
\boldsymbol{N}_{U U}
\end{array}\right] \boldsymbol{\omega}_{U} .
$$

Hence

$$
\boldsymbol{N}\left(C_{0}\right) \boldsymbol{\omega}=\left[\begin{array}{l}
\boldsymbol{N}_{C U} \\
\boldsymbol{N}_{U U}
\end{array}\right] \boldsymbol{\omega}_{U}=\frac{\boldsymbol{\pi}}{\max _{\ell}\left(\pi_{\ell} / \omega_{\ell}\right)},
$$

and the local solution $\boldsymbol{c}^{l o c}\left(\hat{y}(\psi) ; C_{0}\right)$ is given by the formula on the right-hand side of (4.7). It remains to show that this choice of $C$ is the one that yields the lowest values for all elements of $\boldsymbol{c}(0)$, in the case of any large enough value of $\hat{y}(\psi)$.

But we know from Proposition 4 that the solution $\overline{\boldsymbol{c}}(\boldsymbol{\delta})$ is a continuous function of $\boldsymbol{\delta}$; thus there must exist a neighborhood $\mathcal{N}$ of $\mathbf{0}$ such that for any $\boldsymbol{\delta} \in \mathcal{N}$, the solution $\overline{\boldsymbol{c}}(\boldsymbol{\delta})$ has the same set of constrained sectors as the solution for $\boldsymbol{\delta}=\mathbf{0}$. And when $\boldsymbol{\delta}=\mathbf{0}$, the solution to (2.35) is the expenditure vector characterized in Proposition 3, for which the set of constrained sectors is $C_{0}$. Thus for any $\boldsymbol{\delta} \in \mathcal{N}$, the choice of $C$ that results in the lowest value for all of the elements of $\boldsymbol{c}(0)$ must be $C_{0}$.

It follows that in the solution (4.6), $\boldsymbol{c}(0)$ must be given by the local solution corresponding to the choice $C=C_{0}$ (i.e., by the right-hand side of (4.7)) for all values of $\psi$ such that $(\bar{y} / \hat{y}(\psi)) \boldsymbol{\delta} \in \mathcal{N}$. We have already shown that $\hat{y}(\psi)$ can be made arbitrarily large by choosing a small enough value of $\psi$. Hence we can choose a $\psi>0$ such that $\psi<\psi$ implies that $(\bar{y} / \hat{y}(\psi)) \boldsymbol{\delta} \in \mathcal{N}$. This is then a bound such that the solution for $\boldsymbol{c}(0)$ must be the one given in (4.7) for any $\psi<\underline{\psi}$, as asserted in the corollary. 


\section{D.5 Proof of Corollary 7}

The proof follows the same lines as the proof of Corollary 5. It follows from Proposition 9 that the allocation of resources in periods $t \geq 1$ is independent of the choice of $\psi$. Hence (1.7) is again maximized by the policy that maximizes the single-period welfare criterion (D.1), and we can again reduce the problem of choosing $\psi$ to maximize (1.7) to the problem of choosing $\hat{y}$ to maximize (D.1). The main new complication that arises here comes from the fact that now the set of borrowing-constrained sectors can change as we vary $\psi$, for a given specification of $\boldsymbol{\delta}$.

Corollary 6 implies that each of the quantities $\left\{c^{j}(0)\right\}$ and $\left\{y_{j}(0)\right\}$ is a non-decreasing, concave function of $\hat{y}$; and whenever $\psi$ increases, at least one of the $\left\{c^{j}(0)\right\}$ and at least one of the $\left\{y_{j}(0)\right\}$ must increase. It then follows from (D.1) that $W_{0}$ must be a strictly concave function of $\psi$. It is no longer the case that the derivative $\partial W_{0} / \partial \hat{y}$ must be continuous at all points; but $W_{0}$ must have well-defined left and right derivatives at all points, and these must be equal at all but some finite number of values of $\psi$ (the ones at which the set of borrowing-constrained sectors changes). The strict concavity of $W_{0}(\psi)$ continues to imply that the function must have a unique maximum. This must furthermore be the unique point consistent with the first-order condition: $W_{0}$ is maximized at $\psi$ if (a) either the left or right derivative $\partial W_{0} / \partial \hat{y}$ is equal to zero at $\psi$, or (b) the left derivative is greater than zero at $\psi$ while the right derivative is less than zero.

Differentiating (D.1) with respect to $\hat{y}$, we again obtain (D.2). This expression is correct for either the left or right derivative of $W_{0}$, as long as one understands each of the to be correspondingly left or right derivatives. We wish to evaluate the sign of both the left and right derivative at the value $\psi=1$.

Let $C$ and $U$ be the sets of constrained and unconstrained sectors respectively, in the solution (2.38) when $\psi=1$ and $\boldsymbol{\delta}$ has the specified value. For any $j \in C$, we must have

$$
c^{j}(0)=\frac{\delta^{j}}{\bar{p}}+\sum_{k} A_{j k} c^{k}(0)<\omega_{j} \hat{y}(\psi)
$$

when $\psi=1$, and since the solution is continuous in $\psi$, this will continue to be true for all $\psi$ in a neighborhood of 1 . Under the hypothesis that $\boldsymbol{A}_{C U}=\mathbf{0}$, the equality reduces to

$$
c^{j}(0)=\frac{\delta^{j}}{\bar{p}}+\sum_{k \in C} A_{j k} c^{k}(0)
$$

for each $j \in C$. As discussed in the proof of Lemma 6, this system of equations can be uniquely solved for values

$$
c^{j}(0)=\frac{1}{\bar{p}} \boldsymbol{e}_{j}^{\prime} \boldsymbol{M}_{C C} \hat{\boldsymbol{\delta}} .
$$

This will be the solution for all values of $\psi$ in the neighborhood of 1 where these sectors continue to be borrowing-constrained.

Hence we have $\partial c^{j}(0) / \partial \hat{y}=0$ for all $j \in C$, as both the left and right derivative at any $\psi$ in the neighborhood of 1 . It follows that near 1 we can express (D.2) more simply as

$$
\frac{\partial W_{0}}{\partial \hat{y}}=\sum_{j \in U}\left\{\left[u^{\prime}\left(\frac{c^{j}(0)}{\omega_{j}} ; \bar{\xi}\right)-\nu(\bar{\xi})\right] \frac{\partial c^{j}(0)}{\partial \hat{y}}\right\} .
$$


Again, the fact that (4.2) holds with equality for every sector $j \in U$ allows us to write this in the simpler form (D.3).

The derivatives $\partial c^{j}(0) / \partial \hat{y}$ are all non-negative, and there must be at least one sector $j \in U$ for which both the left and right derivative are positive; hence the sum of the $\partial c^{j}(0) / \partial \hat{y}$ terms must be positive, whether a left or right derivative is considered. Thus regardless of whether we consider the left or right derivative, the sign of $\partial W_{0} / \partial \hat{y}$ must be the same as the sign of $u^{\prime}(\hat{y} ; \bar{\xi})-\nu(\bar{\xi})$. This is positive if $\hat{y}<\bar{y}$, negative if $\hat{y}>\bar{y}$, and exactly equal to zero if and only if $\hat{y}=\bar{y}$ exactly.

From this we again conclude that $W_{0}$ is (uniquely) maximized when $\psi$ is chosen so that $\hat{y}(\psi)=\bar{y}$, which holds if and only if $\psi=1$ (the policy assumed in Proposition 4). 